\title{
Double constructions of Frobenius algebras, Connes cocycles and their duality
}

\author{
Chengming Bai
}

\begin{abstract}
We construct an associative algebra with a decomposition into the direct sum of the underlying vector spaces of another associative algebra and its dual space such that both of them are subalgebras and the natural symmetric bilinear form is invariant or the natural antisymmetric bilinear form is a Connes cocycle. The former is called a double construction of a Frobenius algebra and the latter is called a double construction of the Connes cocycle, which is interpreted in terms of dendriform algebras. Both of them are equivalent to a kind of bialgebras, namely, antisymmetric infinitesimal bialgebras and dendriform D-bialgebras, respectively. In the coboundary cases, our study leads to what we call associative Yang-Baxter equation in an associative algebra and $D$-equation in a dendriform algebra, respectively, which are analogues of the classical Yang-Baxter equation in a Lie algebra. We show that an antisymmetric solution of the associative Yang-Baxter equation corresponds to the antisymmetric part of a certain operator called $\mathcal{O}$-operator which gives a double construction of a Frobenius algebra, whereas a symmetric solution of the $D$-equation corresponds to the symmetric part of an $\mathcal{O}$-operator which gives a double construction of the Connes cocycle. By comparing antisymmetric infinitesimal bialgebras and dendriform D-bialgebras, we observe that there is a clear analogy between them. Due to the correspondences between certain symmetries and antisymmetries appearing in this analogy, we regard it as a kind of duality.
\end{abstract}

Mathematics Subject Classification (2010). 16W30, 17A30, 17B60, 57R56, 81T45.

Keywords. Associative algebra, Frobenius algebra, Connes cocycle, Yang-Baxter equation.

\section{Contents}

1 Introduction . . . . . . . . . . . . . . . . . . . . 476

2 Double constructions of Frobenius algebras and another approach to associative Yang-Baxter equation . . . . . . . . . . . . . . . . . . . . . 480

3 Dendriform algebras . . . . . . . . . . . . . . . . . . . . 493

4 Double constructions of Connes cocycles and an analogue of the classical YangBaxter equation . . . . . . . . . . . . . . . . . . . 498

5 Comparison (duality) between bialgebra structures . . . . . . . . . . . . . . 516

Appendix: Some properties of Lie bialgebras and pre-Lie bialgebras . . . . . . . . . . 524

References . . . . . . . . . . . . . . . . . . . . . . . 527 


\section{Introduction}

Throughout this article, an associative algebra is a non-unital associative algebra. There are two important (non-degenerate) bilinear forms on an associative algebra given as follows.

Definition 1.0.1. A bilinear form $\mathcal{B}($, ) on an associative algebra $A$ is invariant if

$$
\mathscr{B}(x y, z)=\mathscr{B}(x, y z) \text { for all } x, y, z \in A .
$$

Definition 1.0.2. An antisymmetric bilinear form $\omega($, ) on an associative algebra $A$ is a cyclic 1-cocycle in the sense of Connes if

$$
\omega(x y, z)+\omega(y z, x)+\omega(z x, y)=0 \quad \text { for all } x, y, z \in A .
$$

We also call for abbreviation $\omega$ a Connes cocycle.

1.1. Frobenius algebras. A Frobenius algebra $(A, \mathscr{B})$ is an associative algebra $A$ with a non-degenerate invariant bilinear form $\mathcal{B}($, ). It was first studied by Frobenius ([Fro]) in 1903 and then named by Brauer and Nesbitt ([BrN]). In fact, Frobenius algebras appear in many fields in mathematics and mathematical physics, such as (modular) representations of finite groups ([Kap]), Hopf algebras ([LS]), statistical models over 2-dimensional graphs ([BFN]), Yang-Baxter equation ([St]), Poisson brackets of hydrodynamic type ([BaN]) and so on. In particular, they play a key role in the study of topological quantum field theory ([Ko], [RFFS], etc.). There are many references concerning the study of Frobenius algebras (for example, see [Kap] or [Y] and the references therein).

A Frobenius algebra $(A, \mathscr{B})$ is symmetric if $\mathscr{B}$ is symmetric. In this article, we mainly consider a class of symmetric Frobenius algebras $(A, \mathscr{B})$ satisfying the conditions

(1) $A=A_{1} \oplus A_{1}^{*}$ as the direct sum of vector spaces;

(2) $A_{1}$ and $A_{1}^{*}$ are associative subalgebras of $A$;

(3) $B$ is the natural symmetric bilinear form on $A_{1} \oplus A_{1}^{*}$ given by

$$
\mathcal{B}\left(x+a^{*}, y+b^{*}\right)=\left\langle x, b^{*}\right\rangle+\left\langle a^{*}, y\right\rangle \text { for all } x, y \in A_{1}, a^{*}, b^{*} \in A_{1}^{*},
$$

where $\langle$,$\rangle is the natural pair between the vector space A_{1}$ and its dual space $A_{1}^{*}$. We call it a double construction of a Frobenius algebra.

Such a double construction of a Frobenius algebra is quite different from the "double extension construction" of a Lie algebra with a non-degenerate invariant bilinear form ([Kac], [MR1]-[MR2], etc.) or the " $T^{*}$-extension" of Frobenius algebra given by Bordemann in [Bo].

Moreover, the above double constructions of Frobenius algebras were also considered by Zhelyabin in [Z] and Aguiar in [A3] (under the name of "balanced Drinfeld 
double $D_{b}(A)$ ") with different motivations and approaches respectively. They are closely related to Lie bialgebras. Lie bialgebras were introduced by Drinfeld ([D]) and play a crucial role in symplectic geometry and quantum groups. They are equivalent to Manin triples (see [CP] and the references therein or Section 5.2).

It is easy to show that the commutator of a Frobenius algebra from the above double construction gives a Manin triple (hence a Lie bialgebra). Furthermore, such a double construction has many properties similar to a Lie bialgebra. It is equivalent to an antisymmetric infinitesimal bialgebra (which also goes under the names of "associative D-algebra" in [Z] and "balanced infinitesimal bialgebra" in the sense of the opposite algebra in [A3]), and under a "coboundary" condition it leads to an analogue of the classical Yang-Baxter equation ([Se]) in an associative algebra $A_{1}$,

$$
r_{12} r_{13}+r_{13} r_{23}-r_{23} r_{12}=0
$$

where $r=\sum_{i} x_{i} \otimes y_{i} \in A_{1} \otimes A_{1}$ and

$$
\begin{aligned}
& r_{12} r_{13}=\sum_{i, j} x_{i} x_{j} \otimes y_{i} \otimes y_{j}, \\
& r_{13} r_{23}=\sum_{i, J} x_{i} \otimes x_{j} \otimes y_{i} y_{j}, \\
& r_{23} r_{12}=\sum_{i, j} x_{j} \otimes x_{i} y_{j} \otimes y_{i} .
\end{aligned}
$$

In particular, an antisymmetric solution of the above equation in $A_{1}$ gives a double construction of a Frobenius algebra $\left(A=A_{1} \oplus A_{1}^{*}, \mathcal{B}\right)$.

On the other hand, we introduce the new notion of antisymmetric infinitesimal bialgebra in order to express explicitly its relation with the known notion of infinitesimal bialgebra, although there are certain notions for the same or similar structures. An infinitesimal bialgebra is a triple $(A, m, \Delta)$, where $(A, m)$ is an associative algebra, $(A, \Delta)$ is a coassociative algebra and

$$
\Delta(a b)=\sum a b_{1} \otimes b_{2}+\sum a_{1} \otimes a_{2} b \quad \text { for all } a, b \in A .
$$

It was introduced by Join and Rota ([JR]) in order to provide an algebraic framework for the calculus of divided difference. Furthermore, Aguiar studied the cases of principal derivations and introduced the associative Yang-Baxter equation ([A1])

$$
r_{13} r_{12}-r_{12} r_{23}+r_{23} r_{13}=0 .
$$

Note that eq. (3) is eq. (6) in the opposite algebra and, when $r$ is antisymmetric, eq. (6) is just eq. (3) under the operation $\sigma_{13}(x \otimes y \otimes z)=z \otimes y \otimes x$.

We would like to point out that although many results on the double constructions of Frobenius algebras have been obtained, a complete and explicit interpretation does not yet exist. In fact, most of these results were given in a scattered way with different motivations. For example, Zhelyabin in [Z] introduced the notion of associative Dalgebra as an important step to develop a bialgebra theory of Jordan algebras (an 
explicit study of coboundary cases for the associative algebras themselves is not performed). In [A3], Aguiar introduced the notion of balanced infinitesimal bialgebra and then studied the antisymmetric solutions of eq. (6) in order to compare them with Lie bialgebras and the classical Yang-Baxter equation in a Lie algebra, respectively, and the balanced Drinfeld double $D_{b}(A)$ appears as an important consequence. We will formulate the known results by a different and systematic approach (for example, the "invariant" antisymmetry appears naturally). Moreover such an approach is useful and convenient for the whole study in this article.

1.2. $\mathcal{O}$-operators and dendriform algebras. When $r$ is antisymmetric, besides the standard tensor form (3) or (6), the associative Yang-Baxter equation has an equivalent operator form, that is, a special case of a certain operator called $\mathcal{O}$-operator. An $\mathcal{O}$ operator associated to a bimodule $(l, r, V)$ of an associative algebra $A$ is a linear map $T: V \rightarrow A$ satisfying

$$
T(u) \cdot T(v)=T(l(T(u)) v+r(T(v) u)) \quad \text { for all } u, v \in V .
$$

In fact, an antisymmetric solution of the associative Yang-Baxter equation is an $\mathcal{O}$-operator associated to the bimodule $\left(R^{*}, L^{*}\right)$. The notion of $\mathcal{O}$-operator was introduced in [BGN1] (such a structure appeared independently in [U] under the name of generalized Rota-Baxter operator), which is an analogue of the $\mathcal{O}$-operator defined by Kupershmidt as a natural generalization of the operator form of the classical Yang-Baxter equation ([Ku3] and a further study in [Bai1]). Conversely, the antisymmetric part of an $\mathcal{O}$-operator satisfies the associative Yang-Baxter equation in a larger associative algebra.

From an $\mathcal{O}$-operator, one can get a dendriform algebra. Dendriform algebras are equipped with an associative product which can be written as a linear combination of nonassociative compositions. They were introduced by Loday ([Lo1]) with motivation from algebraic K-theory and have been studied quite extensively with connections to several areas in mathematics and physics, including operads ([Lo3]), homology ([Fra1]-[Fra2]), Hopf algebras ([Cha2], [H1]-[H2], [Ron], [LR2]), Lie and Leibniz algebras ([Fra2]), combinatorics ([LR1]), arithmetic ([Lo2]) and quantum field theory ([F1]) and so on (see [EMP] and the references therein).

Furthermore, there is a compatible dendriform algebra structure on an associative algebra $A$ if and only if there exists an invertible $\mathcal{O}$-operator of $A$, or equivalently, there exists an invertible (usual) 1-cocycle (see eq. (34)) associated to certain suitable bimodule of $A$ ([BGN2]). Thus a close relation between the associative Yang-Baxter equation (hence the antisymmetric infinitesimal bialgebras and the double construction of Frobenius algebras) and dendriform algebras is obviously given (see also [A3], [E1]-[E2]).

1.3. Connes cocycles. Note that a Connes cocycle given by eq. (1) is in fact a Hochschild 2-cocycle which satisfies antisymmetry. It corresponds to the original 
definition of cyclic cohomology by Connes ([C]). Also note that in cyclic cohomology a cyclic $n$-cocycle in the sense of Connes is an $(n+1)$-linear form, although a Connes cocycle was called a cyclic 2-cocycle in some references (like [A3]) from some different viewpoints. Moreover, although Connes used it in the unital framework and in the non-unital framework cyclic homology has a very different behavior, we still use the notion of "Connes cocycle" in this article.

We will see that, from a non-degenerate Connes cocycle on an associative algebra $A$, one can get a compatible dendriform algebra structure on $A$. Moreover, the dendriform algebra structures play a key role in the following constructions of nondegenerate Connes cocycles, which is one of the main issues of this article. We call $(A, \omega)$ a double construction of the Connes cocycle if it satisfies the conditions

(1) $A=A_{1} \oplus A_{1}^{*}$ as the direct sum of vector spaces;

(2) $A$ is an associative algebra and $A_{1}$ and $A_{1}^{*}$ are associative subalgebras of $A$;

(3) $\omega$ is the natural antisymmetric bilinear form on $A_{1} \oplus A_{1}^{*}$ given by

$$
\omega\left(x+a^{*}, y+b^{*}\right)=-\left\langle x, b^{*}\right\rangle+\left\langle a^{*}, y\right\rangle \text { for all } x, y \in A_{1}, a^{*}, b^{*} \in A_{1}^{*},
$$

and $\omega$ is a Connes cocycle on $A$.

In this article, the double construction of the Connes cocycle is interpreted in terms of dendriform algebras. We find that such a structure is quite similar to a double construction of a Frobenius algebra or a Lie bialgebra. Briefly speaking, a double construction of the Connes cocycle is equivalent to a certain bialgebra structure, namely, a dendriform D-bialgebra structure. Both antisymmetric infinitesimal bialgebras and dendriform D-bialgebras have many similar properties as Lie bialgebras. In particular, there are the so-called coboundary dendriform D-bialgebras which lead to another analogue ( $D$-equation in a dendriform algebra) of the classical Yang-Baxter equation. A symmetric solution of the $D$-equation corresponds to the symmetric part of an $\mathcal{O}$-operator, which gives a double construction of the Connes cocycle.

1.4. Duality between bialgebras. By comparing antisymmetric infinitesimal bialgebras and dendriform D-bialgebras, we observe that there is a clear analogy between them. Moreover, due to the correspondences between certain symmetries and antisymmetries appearing in the analogy, we regard it as a kind of duality.

There is a similar study in the version of Lie algebras ([CP], [Bai2]). In fact, there is also a double construction of a Lie algebra with a non-degenerate invariant bilinear form (Manin triple or Lie bialgebra) or with a non-degenerate 2-cocycle of Lie algebra (para-Kähler Lie algebra or pre-Lie bialgebra). There are the $\mathcal{O}$ operators and a kind of algebras called pre-Lie algebras (Lie-admissible algebras whose left multiplication operators form a Lie algebra) which play the same role as the $\mathcal{O}$-operators and dendriform algebras. And there is a similar duality between Lie bialgebras and pre-Lie bialgebras. 
Moreover, due to Chapoton ([Cha1]), there is a close relationship among the Lie algebras, associative algebras, pre-Lie algebras and dendriform algebras that can be depicted by a commutative diagram of categories:

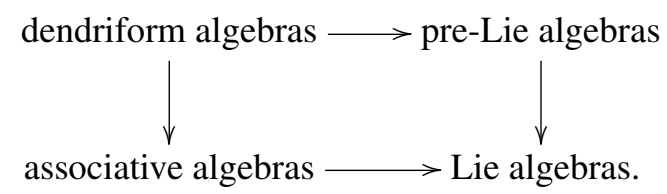

We will extend the above relationship at the level of bialgebras with the dualities in a commutative diagram. In particular, the relation between antisymmetric infinitesimal bialgebras (the special case of infinitesimal Hopf algebras) and Lie bialgebras was observed in [A3]. Furthermore, these types of bialgebras fit into the general framework of "generalized bialgebras", as introduced by Loday in [Lo4].

The article is organized as follows. In Section 2, we give an explicit and systematic study on the double constructions of Frobenius algebras and then obtain the associative Yang-Baxter equation naturally. In Section 3, we introduce the close relations between $\mathcal{O}$-operators and dendriform algebras. In Section 4 , we study the double constructions of Connes cocycles in terms of dendriform algebras. In Section 5, we give a clear analogy between antisymmetric infinitesimal bialgebras and dendriform D-bialgebras, which we regard as a kind of duality. After recalling a similar duality between Lie bialgebras and pre-Lie bialgebras, we express a close relationship among associative algebras, Lie algebras, pre-Lie algebras and dendriform algebras at the level of bialgebras.

Throughout this article, all algebras are finite-dimensional, although many results still hold in the infinite-dimensional case.

\section{Double constructions of Frobenius algebras and another approach to asso- ciative Yang-Baxter equation}

\subsection{Bimodules and matched pairs of associative algebras}

Definition 2.1.1. Let $A$ be an associative algebra and let $V$ be a vector space. Let $l, r: A \rightarrow \mathfrak{g} \mathfrak{l}(V)$ be two linear maps. $V$ (or the pair $(l, r)$, or $(l, r, V)$ ) is called a bimodule of $A$ if

$$
l(x y) v=l(x) l(y) v, \quad r(x y) v=r(y) r(x) v, \quad l(x) r(y) v=r(y) l(x) v
$$

for all $x, y \in A, v \in V$.

In fact, according to [Sc], $(l, r, V)$ is a bimodule of an associative algebra $A$ if and only if the direct sum $A \oplus V$ of vector spaces is turned into an associative algebra (the semidirect sum) by defining multiplication in $A \oplus V$ by

$$
\left(x_{1}+v_{1}\right) *\left(x_{2}+v_{2}\right)=x_{1} \cdot x_{2}+\left(l\left(x_{1}\right) v_{2}+r\left(x_{2}\right) v_{1}\right)
$$


for all $x_{1}, x_{2} \in A, v_{1}, v_{2} \in V$. We denote it by $A \ltimes_{l, r} V$ or simply $A \ltimes V$.

The following conclusion is obvious.

Lemma 2.1.2. Let $(l, r, V)$ be a bimodule of an associative algebra $A$.

(1) Let $l^{*}, r^{*}: A \rightarrow \mathfrak{g l}\left(V^{*}\right)$ be the linear maps given by

$$
\left\langle l^{*}(x) u^{*}, v\right\rangle=\left\langle l(x) v, u^{*}\right\rangle, \quad\left\langle r^{*}(x) u^{*}, v\right\rangle=\left\langle r(x) v, u^{*}\right\rangle
$$

for all $x \in A, u^{*} \in V^{*}, v \in V$. Then $\left(r^{*}, l^{*}, V^{*}\right)$ is a bimodule of $A$.

(2) $(l, 0, V),(0, r, V),\left(r^{*}, 0, V^{*}\right)$ and $\left(0, l^{*}, V^{*}\right)$ are bimodules of $A$.

Example 2.1.3. Let $A$ be an associative algebra. Let $L(x)$ and $R(x)$ denote the left and right multiplication operator, respectively, that is, $L(x)(y)=x y, R(x)(y)=y x$ for any $x, y \in A$. Let $L: A \rightarrow \operatorname{gl}(A)$ with $x \rightarrow L(x)$ and $R: A \rightarrow \operatorname{gl}(A)$ with $x \rightarrow R(x)$ (for every $x \in A)$ be two linear maps. Then $(L, 0),(0, R)$ and $(L, R)$ are bimodules of $A$. On the other hand, $\left(R^{*}, 0\right),\left(0, L^{*}\right)$ and $\left(R^{*}, L^{*}\right)$ are bimodules of $A$, too.

Theorem 2.1.4. Let $(A, \cdot)$ and $(B, \circ)$ be two associative algebras. Suppose that there are linear maps $l_{A}, r_{A}: A \rightarrow \mathfrak{g l}(B)$ and $l_{B}, r_{B}: B \rightarrow \mathfrak{g l}(A)$ such that $\left(l_{A}, r_{A}\right)$ is a bimodule of $A$ and $\left(l_{B}, r_{B}\right)$ is a bimodule of $B$ and they satisfy the following conditions:

$$
\begin{gathered}
l_{A}(x)(a \circ b)=l_{A}\left(r_{B}(a) x\right) b+\left(l_{A}(x) a\right) \circ b, \\
r_{A}(x)(a \circ b)=r_{A}\left(l_{B}(b) x\right) a+a \circ\left(r_{A}(x) b\right), \\
l_{B}(a)(x \cdot y)=l_{B}\left(r_{A}(x) a\right) y+\left(l_{B}(a) x\right) \cdot y, \\
r_{B}(a)(x \cdot y)=r_{B}\left(l_{A}(y) a\right) x+x \cdot\left(r_{B}(a) y\right), \\
l_{A}\left(l_{B}(a) x\right) b+\left(r_{A}(x) a\right) \circ b-r_{A}\left(r_{B}(b) x\right) a-a \circ\left(l_{A}(x) b\right)=0, \\
l_{B}\left(l_{A}(x) a\right) y+\left(r_{B}(a) x\right) \cdot y-r_{B}\left(r_{A}(y) a\right) x-x \cdot\left(l_{B}(a) y\right)=0
\end{gathered}
$$

for any $x, y \in A, a, b \in B$. Then there is an associative algebra structure on the direct sum $A \oplus B$ of the underlying vector spaces of $A$ and $B$ given by

$$
(x+a) *(y+b)=\left(x \cdot y+l_{B}(a) y+r_{B}(b) x\right)+\left(a \circ b+l_{A}(x) b+r_{A}(y) a\right)
$$

for all $x, y \in A, a, b \in B$. We denote this associative algebra by $A \bowtie_{l_{B}, r_{B}}^{l_{A}, r_{A}} B$ or simply $A \bowtie B$. On the other hand, every associative algebra with a decomposition into the direct sum of the underlying vector spaces of two subalgebras can be obtained in this way.

Proof. This is straightforward.

Definition 2.1.5. Let $(A, \cdot)$ and $(B, \circ)$ be two associative algebras. Suppose that there are linear maps $l_{A}, r_{A}: A \rightarrow \mathfrak{g l}(B)$ and $l_{B}, r_{B}: B \rightarrow \mathfrak{g l}(A)$ such that $\left(l_{A}, r_{A}\right)$ is a bimodule of $A$ and $\left(l_{B}, r_{B}\right)$ is a bimodule of $B$. If eqs. (9)-(14) are satisfied, then $\left(A, B, l_{A}, r_{A}, l_{B}, r_{B}\right)$ is called a matched pair of associative algebras. 
Remark 2.1.6. Obviously $B$ is an ideal of $A \bowtie B$ if and only if $l_{B}=r_{B}=0$. If $B$ is a trivial (that is, all the products of $B$ are zero) ideal, then $A \bowtie_{0,0}^{l_{A}, r_{A}} B \cong A \ltimes_{l_{A}, r_{A}} B$. Moreover, some other special cases of Theorem 2.1.4 have already been studied. For example, the case when $A$ is a left $B$-module and $B$ is a right $A$-module was considered in [A1], that is, $l_{A}=0$ and $r_{B}=0$.

2.2. Double constructions of Frobenius algebras and antisymmetric infinitesimal bialgebras. Recall that a (symmetric) Frobenius algebra is an associative algebra $A$ with a non-degenerate (symmetric) invariant bilinear form. Let $(A, \cdot)$ be an associative algebra. Suppose that there is an associative algebra structure "o" on its dual space $A^{*}$. We construct an associative algebra structure on the direct sum $A \oplus A^{*}$ of the underlying vector spaces of $A$ and $A^{*}$ such that $(A, \cdot)$ and $\left(A^{*}, \circ\right)$ are subalgebras and the symmetric bilinear form on $A \oplus A^{*}$ given by eq. (2) is invariant. That is, $\left(A \oplus A^{*}, \mathcal{B}\right)$ is a symmetric Frobenius algebra. Such a construction is called a double construction of a Frobenius algebra associated to $(A, \cdot)$ and $\left(A^{*}, \circ\right)$ and we denote it by $\left(A \bowtie A^{*}, \mathscr{B}\right)$.

Theorem 2.2.1. Let $(A, \cdot)$ be an associative algebra. Suppose that there is an associative algebra structure "०" on its dual space $A^{*}$. Then there is a double construction of a Frobenius algebra associated to $(A, \cdot)$ and $\left(A^{*}, \circ\right)$ if and only if $\left(A, A^{*}, R_{.}^{*}, L_{.}^{*}, R_{\circ}^{*}, L_{\circ}^{*}\right)$ is a matched pair of associative algebras.

Proof. If $\left(A, A^{*}, R_{.}^{*}, L_{.}^{*}, R_{\circ}^{*}, L_{\circ}^{*}\right)$ is a matched pair of associative algebras, then it is straightforward to show that the bilinear form (2) is invariant on the associative algebra $A \bowtie_{R_{\circ}^{*}, L_{\circ}^{*}}^{R_{*}^{*}, L^{*}} A^{*}$ given by eq. (15). Conversely, set

$$
x * a^{*}=l_{A}(x) a^{*}+r_{A^{*}}\left(a^{*}\right) x, \quad a^{*} * x=l_{A^{*}}\left(a^{*}\right) x+r_{A}(x) a^{*}
$$

for all $x \in A, a^{*} \in A^{*}$. Then $\left(A, A^{*}, l_{A}, r_{A}, l_{A^{*}}, r_{A^{*}}\right)$ is a matched pair of associative algebras. Note that

$$
\begin{gathered}
\left\langle l_{A}(x) a^{*}, y\right\rangle=\left\langle r_{A}(y) a^{*}, x\right\rangle=\left\langle y \cdot x, a^{*}\right\rangle, \\
\left\langle l_{A^{*}}\left(b^{*}\right) x, a^{*}\right\rangle=\left\langle r_{A^{*}}\left(a^{*}\right) x, b^{*}\right\rangle=\left\langle a^{*} \circ b^{*}, x\right\rangle,
\end{gathered}
$$

where $x, y \in A, a^{*}, b^{*} \in A^{*}$. Hence, $l_{A}=R_{.}^{*}, r_{A}=L_{.}^{*}, l_{A^{*}}=R_{\circ}^{*}, r_{A^{*}}=L_{\circ}^{*}$.

Proposition 2.2.2. Let $(A, \cdot)$ be an associative algebra. Suppose that there is an associative algebra structure “o” on its dual space $A^{*}$. Then $\left(A, A^{*}, R_{.}^{*}, L_{.}^{*}, R_{\circ}^{*}, L_{\circ}^{*}\right)$ is a matched pair of associative algebras if and only if for any $x \in A^{*}, a^{*}, b^{*} \in A^{*}$,

$$
\begin{aligned}
R_{.}^{*}(x)\left(a^{*} \circ b^{*}\right) & =R_{.}^{*}\left(L_{\circ}^{*}\left(a^{*}\right) x\right) b^{*}+\left(R_{.}^{*}(x) a^{*}\right) \circ b^{*}, \\
R_{.}^{*}\left(R_{\circ}^{*}\left(a^{*}\right) x\right) b^{*}+L_{.}^{*}(x) a^{*} \circ b^{*} & =L_{.}^{*}\left(L_{\circ}^{*}\left(b^{*}\right) x\right) a^{*}+a^{*} \circ\left(R_{.}^{*}(x) b^{*}\right) .
\end{aligned}
$$


Proof. Obviously, eq. (16) is just eq. (9) and eq. (17) is just eq. (13) in the case when $l_{A}=R_{.}^{*}, r_{A}=L_{.}^{*}, l_{B}=l_{A^{*}}=R_{\circ}^{*}, r_{B}=r_{A^{*}}=L_{\circ}^{*}$. By eq. (8), it is easy to show that in this situation

$$
\begin{aligned}
\text { eq. (9) } \Longleftrightarrow \text { eq. (10) } \Longleftrightarrow \text { eq. (11) } \Longleftrightarrow \text { eq. (12), } \\
\text { eq. (13) } \Longleftrightarrow \text { eq. (14). }
\end{aligned}
$$

Therefore the conclusion holds.

Before the next study, we give some notations as follows. Let $A$ be an associative algebra. Let $\sigma: A \otimes A \rightarrow A \otimes A$ be the exchange operator defined as

$$
\sigma(x \otimes y)=y \otimes x \quad \text { for all } x, y \in A .
$$

There are several ways to make $A \otimes A$ into a bimodule of $A$. For example, let id be the identity map on $A$. Then (id $\otimes L, R \otimes$ id) given by (for any $x, a, b \in A$ )

$$
\begin{aligned}
& (\mathrm{id} \otimes L)(x)(a \otimes b)=(\mathrm{id} \otimes L(x))(a \otimes b)=a \otimes x b, \\
& (R \otimes \mathrm{id})(x)(a \otimes b)=(R(x) \otimes \mathrm{id})(a \otimes b)=a x \otimes b,
\end{aligned}
$$

is a bimodule of $A$. Similarly, $(L \otimes \mathrm{id}$, id $\otimes R)$ is also a bimodule of $A$. In fact, eq. (5) given in the introduction can be rewritten as

$$
\Delta(a b)=(L(a) \otimes \mathrm{id}) \Delta(b)+(\mathrm{id} \otimes R(b)) \Delta(a),
$$

which gives the notion of infinitesimal bialgebra ([JR]).

For a linear map $\phi: V_{1} \rightarrow V_{2}$, we denote the dual (linear) map by $\phi^{*}: V_{2}^{*} \rightarrow V_{1}^{*}$ given by

$$
\left\langle v, \phi^{*}\left(u^{*}\right)\right\rangle=\left\langle\phi(v), u^{*}\right\rangle \quad \text { for all } v \in V_{1}, u^{*} \in V_{2} .
$$

Theorem 2.2.3. Let $(A, \cdot)$ be an associative algebra. Suppose there is an associative algebra structure "o" on its dual space $A^{*}$ given by a linear map $\Delta^{*}: A^{*} \otimes A^{*} \rightarrow A^{*}$. Then $\left(A, A^{*}, R_{.}^{*}, L_{.}^{*}, R_{\circ}^{*}, L_{\circ}^{*}\right)$ is a matched pair of associative algebras if and only if $\Delta: A \rightarrow A \otimes A$ satisfies the following two conditions:

$$
\Delta(x \cdot y)=(\mathrm{id} \otimes L \cdot(x)) \Delta(y)+(R .(y) \otimes \mathrm{id}) \Delta(x),
$$

$(L .(y) \otimes \mathrm{id}-\mathrm{id} \otimes R .(y)) \Delta(x)+\sigma[(L .(x) \otimes \mathrm{id}-\mathrm{id} \otimes R .(x)) \Delta(y)]=0$

for all $x, y \in A$.

Proof. Let $\left\{e_{1}, \ldots, e_{n}\right\}$ be a basis of $A$ and let $\left\{e_{1}^{*}, \ldots, e_{n}^{*}\right\}$ be its dual basis. Set $e_{i} \cdot e_{j}=\sum_{k=1}^{n} c_{i j}^{k} e_{k}$ and $e_{i}^{*} \circ e_{j}^{*}=\sum_{k=1}^{n} f_{i j}^{k} e_{k}^{*}$. Therefore, we have $\Delta\left(e_{k}\right)=$ 
$\sum_{i, j=1}^{n} f_{i j}^{k} e_{i} \otimes e_{j}$ and

$$
\begin{array}{ll}
R_{.}^{*}\left(e_{i}\right) e_{j}^{*}=\sum_{k=1}^{n} c_{k i}^{j} e_{k}^{*}, & L_{.}^{*}\left(e_{i}\right) e_{j}^{*}=\sum_{k=1}^{n} c_{i k}^{j} e_{k}^{*}, \\
R_{\circ}^{*}\left(e_{i}^{*}\right) e_{j}=\sum_{k=1}^{n} f_{k i}^{j} e_{k}, & L_{\circ}^{*}\left(e_{i}^{*}\right) e_{j}=\sum_{k=1}^{n} f_{i k}^{j} e_{k} .
\end{array}
$$

Hence the coefficient of $e_{j} \otimes e_{k}$ in

$$
\Delta\left(e_{i} \cdot e_{m}\right)=\left(\mathrm{id} \otimes L .\left(e_{i}\right)\right) \Delta\left(e_{m}\right)+\left(R .\left(e_{m}\right) \otimes \mathrm{id}\right) \Delta\left(e_{i}\right)
$$

gives the relation (for any $i, j, k, m$ )

$$
\sum_{l=1}^{n} c_{m i}^{l} f_{j k}^{l}=\sum_{l=1}^{n}\left(c_{m l}^{k} f_{j l}^{i}+c_{l i}^{j} f_{l k}^{m}\right),
$$

which is just the relation given by the coefficient of $e_{m}^{*}$ in

$$
R_{.}^{*}\left(e_{i}\right)\left(e_{j}^{*} \circ e_{k}^{*}\right)=R_{.}^{*}\left(L_{\circ}^{*}\left(e_{j}^{*}\right) e_{i}\right) e_{k}^{*}+\left(R_{.}^{*}\left(e_{i}\right) e_{j}^{*}\right) \circ e_{k}^{*} .
$$

Similarly, eq. (20) corresponds to eq. (17).

Remark 2.2.4. From the symmetry of the associative algebras $(A, \cdot)$ and $\left(A^{*}, \circ\right)$ appearing in the double construction, we also can consider the operation $\beta: A^{*} \rightarrow$ $A^{*} \otimes A^{*}$ such that $\beta^{*}: A \otimes A \rightarrow A$ gives an associative algebra structure on $A$. It is easy to show that $\Delta$ satisfies eqs. (19) and (20) if and only if $\beta$ satisfies

$$
\beta\left(a^{*} \circ b^{*}\right)=\left(\mathrm{id} \otimes L_{\circ}\left(a^{*}\right)\right) \beta\left(b^{*}\right)+\left(R_{\circ}\left(b^{*}\right) \otimes \mathrm{id}\right) \beta\left(a^{*}\right),
$$

$\left(L_{\circ}\left(b^{*}\right) \otimes \mathrm{id}-\mathrm{id} \otimes R_{\circ}\left(b^{*}\right)\right) \beta\left(a^{*}\right)+\sigma\left[\left(L_{\circ}\left(a^{*}\right) \otimes \mathrm{id}-\mathrm{id} \otimes R_{\circ}\left(a^{*}\right)\right) \beta\left(b^{*}\right)\right]=0$

for all $a^{*}, b^{*} \in A$.

Definition 2.2.5. Let $A$ be an associative algebra. An antisymmetric infinitesimal bialgebra structure on $A$ is a linear map $\Delta: A \rightarrow A \otimes A$ such that

(a) $\Delta^{*}: A^{*} \otimes A^{*} \rightarrow A^{*}$ defines an associative algebra structure on $A^{*}$;

(b) $\Delta$ satisfies eqs. (19) and (20).

We denote it by $(A, \Delta)$ or $\left(A, A^{*}\right)$.

Corollary 2.2.6. Let $(A, \cdot)$ and $\left(A^{*}, \circ\right)$ be two associative algebras. Then the following conditions are equivalent.

(1) There is a double construction of a Frobenius algebra associated to $(A, \cdot)$ and $\left(A^{*}, \circ\right)$; 
(2) ( $\left.A, A^{*}, R_{.}^{*}, L_{.}^{*}, R_{\circ}^{*}, L_{\circ}^{*}\right)$ is a matched pair of associative algebras;

(3) $\left(A, A^{*}\right)$ is an antisymmetric infinitesimal bialgebra.

Proof. It follows from Theorems 2.2.1 and 2.2.3.

Remark 2.2.7. As we have pointed out in the introduction, an antisymmetric infinitesimal bialgebra is exactly an associative D-algebra in [Z] where the above equivalence between (1) and (3) was given and a balanced infinitesimal bialgebra in the sense of the opposite algebra in [A3] where the corresponding double construction of a Frobenius algebra was called a balanced Drinfeld double as an important consequence. On the other hand, the notion of antisymmetric infinitesimal bialgebra is due to the fact that eq. (19) (in the sense of the opposite algebra) corresponds to eq. (18) which gives the notion of infinitesimal bialgebra and eq. (20) expresses certain antisymmetry.

Definition 2.2.8. Let $\left(A, \Delta_{A}\right)$ and $\left(B, \Delta_{B}\right)$ be two antisymmetric infinitesimal bialgebras. A homomorphism of antisymmetric infinitesimal bialgebras $\varphi: A \rightarrow B$ is a homomorphism of associative algebras such that

$$
(\varphi \otimes \varphi) \Delta_{A}(x)=\Delta_{B}(\varphi(x)) \text { for all } x \in A .
$$

An isomorphism of antisymmetric infinitesimal bialgebras is an invertible homomorphism of antisymmetric infinitesimal bialgebras.

Definition 2.2.9. Let $\left(A_{1} \bowtie A_{1}^{*}, \mathscr{B}_{1}\right)$ and $\left(A_{2} \bowtie A_{2}^{*}, \mathscr{B}_{2}\right)$ be two double constructions of Frobenius algebras. They are isomorphic if and only if there exists an isomorphism of associative algebras $\varphi: A_{1} \bowtie A_{1}^{*} \rightarrow A_{2} \bowtie A_{2}^{*}$ such that

$$
\varphi\left(A_{1}\right)=A_{2}, \quad \varphi\left(A_{1}^{*}\right)=A_{2}^{*}, \quad \mathscr{B}_{1}(x, y)=\varphi^{*} \mathscr{B}_{2}(x, y)=\mathscr{B}_{2}(\varphi(x), \varphi(y))
$$

for all $x, y \in A_{1} \bowtie A_{1}^{*}$.

Proposition 2.2.10. Two double constructions of Frobenius algebras are isomorphic if and only if their corresponding antisymmetric infinitesimal bialgebras are isomorphic.

Proof. Let $\left(A_{1} \bowtie A_{1}^{*}, \mathscr{B}_{1}\right)$ and $\left(A_{2} \bowtie A_{2}^{*}, \mathscr{B}_{2}\right)$ be two double constructions of Frobenius algebras. Let $\left\{e_{1}, \ldots, e_{n}\right\}$ be a basis of $A_{1}$ and $\left\{e_{1}^{*}, \ldots, e_{n}^{*}\right\}$ its dual basis. If $\varphi: A_{1} \bowtie A_{1}^{*} \rightarrow A_{2} \bowtie A_{2}^{*}$ is an isomorphism of double constructions of Frobenius algebras, then $\left.\varphi\right|_{A_{1}}: A_{1} \rightarrow A_{2}$ and $\left.\varphi\right|_{A_{1}^{*}}: A_{1}^{*} \rightarrow A_{2}^{*}$ are isomorphisms of associative algebras. Moreover, $\left.\varphi\right|_{A_{1}^{*}}=\left(\left.\varphi\right|_{A_{1}}\right)^{*-1}$ since

$$
\begin{aligned}
\left\langle\left.\varphi\right|_{A_{1}^{*}}\left(e_{i}^{*}\right), \varphi\left(e_{j}\right)\right\rangle & =\mathscr{B}_{2}\left(\left.\varphi\right|_{A_{1}^{*}}\left(e_{i}^{*}\right), \varphi\left(e_{j}\right)\right) \\
& =\mathscr{B}_{1}\left(e_{i}^{*}, e_{j}\right)=\delta_{i j}=\left\langle e_{i}^{*}, e_{j}\right\rangle \\
& =\left\langle\varphi^{*}\left(\left.\varphi\right|_{A_{1}}\right)^{*-1}\left(e_{i}^{*}\right), e_{j}\right\rangle=\left\langle\left(\left.\varphi\right|_{A_{1}}\right)^{*-1}\left(e_{i}^{*}\right), \varphi\left(e_{j}\right)\right\rangle .
\end{aligned}
$$


Hence $\left(A_{1}, A_{1}^{*}\right)$ and $\left(A_{2}, A_{2}^{*}\right)$ are isomorphic as antisymmetric infinitesimal bialgebras. Conversely, let $\varphi^{\prime}: A_{1} \rightarrow A_{2}$ be an isomorphism between two antisymmetric infinitesimal bialgebras $\left(A_{1}, A_{1}^{*}\right)$ and $\left(A_{2}, A_{2}^{*}\right)$. Set $\varphi: A_{1} \oplus A_{1}^{*} \rightarrow A_{2} \oplus A_{2}^{*}$ be a linear map given by

$$
\varphi(x)=\varphi^{\prime}(x), \varphi\left(a^{*}\right)=\left(\varphi^{*}\right)^{-1}\left(a^{*}\right) \quad \text { for all } x \in A_{1}, a^{*} \in A_{1}^{*} .
$$

Then it is easy to show that $\varphi$ is an isomorphism of double constructions of Frobenius algebras between $\left(A_{1} \bowtie A_{1}^{*}, \mathscr{B}_{1}\right)$ and $\left(A_{2} \bowtie A_{2}^{*}, \mathscr{B}_{2}\right)$.

Example 2.2.11. Let $(A, \Delta)$ be an antisymmetric infinitesimal bialgebra. Then its dual $\left(A^{*}, \beta\right)$ given in Remark 2.2.4 is also an antisymmetric infinitesimal bialgebra.

Example 2.2.12. Let $A$ be an associative algebra. If the associative algebra structure on $A^{*}$ is trivial, then either $(A, 0)$ or $\left(A, A^{*}\right)$ is an antisymmetric infinitesimal bialgebra. Moreover, its corresponding Frobenius algebra is given by the semidirect sum $A \ltimes_{R^{*}, L^{*}} A^{*}$ with the natural invariant bilinear form $B$ given by eq. (2). Dually, if $A$ is a trivial associative algebra, then the antisymmetric infinitesimal bialgebra structures on $A$ are in one-to-one correspondence with the associative algebra structures on $A^{*}$.

Example 2.2.13. Let $\left(A, A^{*}\right)$ be an antisymmetric infinitesimal bialgebra. In the next subsection, we will prove that there exists a canonical antisymmetric infinitesimal bialgebra structure on the direct sum $A \oplus A^{*}$ of the underlying vector spaces of $A$ and $A^{*}$.

2.3. Coboundary (principal) antisymmetric infinitesimal bialgebras. In fact, for an associative algebra $A, \Delta: A \rightarrow A \otimes A$ satisfying eq. (19) is a 1-cocycle or a derivation of $A$ associated to the bimodule (id $\otimes L, R \otimes$ id). So it is natural to consider the special case that $\Delta$ is a 1-coboundary or a principal derivation.

Definition 2.3.1. An antisymmetric infinitesimal bialgebra $(A, \Delta)$ is called coboundary if there exists a $r \in A \otimes A$ such that

$$
\Delta(x)=(\mathrm{id} \otimes L(x)-R(x) \otimes \mathrm{id}) r \quad \text { for all } x \in A .
$$

Let $A$ be an associative algebra and $r \in A \otimes A$. If $\Delta: A \rightarrow A \otimes A$ is given by eq. (21), then it is obvious that $\Delta$ satisfies eq. (19). Therefore, $(A, \Delta)$ is an antisymmetric infinitesimal bialgebra if and only if the following two conditions are satisfied:

(1) $\Delta^{*}: A^{*} \otimes A^{*} \rightarrow A^{*}$ defines an associative algebra structure on $A^{*}$.

(2) $\Delta$ satisfies eq. (20). 
Lemma 2.3.2 ([A1], Proposition 5.1). Let $A$ be an associative algebra and $r \in A \otimes A$. Define $\Delta: A \rightarrow A \otimes A$ by

$$
\Delta(a)=[L(x) \otimes \mathrm{id}-\mathrm{id} \otimes R(x)] r
$$

for all $x \in A$. Then $\Delta^{*}: A^{*} \otimes A^{*} \rightarrow A^{*}$ defines an associative algebra structure on $A^{*}$ if and only if

$$
(L(x) \otimes \mathrm{id} \otimes \mathrm{id}-\mathrm{id} \otimes \mathrm{id} \otimes R(x))\left(r_{13} r_{12}+r_{23} r_{13}-r_{12} r_{23}\right)=0
$$

for all $x \in A$, where the notations $r_{13} r_{12}, r_{23} r_{13}, r_{12} r_{23}$ are given similarly as in eq. (4).

Therefore for (1), we use a similar discussion to get the following conclusion.

Proposition 2.3.3. Let $A$ be an associative algebra and $r \in A \otimes A$. Define $\Delta: A \rightarrow$ $A \otimes A$ by eq. (21). Then $\Delta^{*}: A^{*} \otimes A^{*} \rightarrow A^{*}$ defines an associative algebra structure on $A^{*}$ if and only if

$(\mathrm{id} \otimes \mathrm{id} \otimes L(x)-R(x) \otimes \mathrm{id} \otimes \mathrm{id})\left(r_{12} r_{13}+r_{13} r_{23}-r_{23} r_{12}\right)=0$

for all $x \in A$.

Proposition 2.3.4. Let $A$ be an associative algebra and $r \in A \otimes A$. Define $\Delta: A \rightarrow$ $A \otimes A$ by eq. (21). Then $\Delta$ satisfies eq. (20) if and only if $r$ satisfies

$$
[L(x) \otimes \mathrm{id}-\mathrm{id} \otimes R(x)][\mathrm{id} \otimes L(y)-R(y) \otimes \mathrm{id}](r+\sigma(r))=0
$$

for all $x, y \in A$.

Proof. This is straightforward.

Combining Proposition 2.3.3 and Proposition 2.3.4, we have the following conclusion.

Theorem 2.3.5. Let $A$ be an associative algebra and $r \in A \otimes A$. Then the linear map $\Delta$ defined by eq. (21) induces an associative algebra structure on $A^{*}$ such that $\left(A, A^{*}\right)$ is an antisymmetric infinitesimal bialgebra if and only if eqs. (22) and (23) are satisfied.

Theorem 2.3.6. Let $\left(A, \Delta_{A}\right)$ be an antisymmetric infinitesimal bialgebra. Then there is a canonical antisymmetric infinitesimal bialgebra structure on the direct sum $A \oplus A^{*}$ of the underlying vector spaces of $A$ and $A^{*}$ such that both the inclusions $i_{1}: A \rightarrow A \oplus A^{*}$ and $i_{2}: A^{*} \rightarrow A \oplus A^{*}$ into the two summands are homomorphisms of antisymmetric infinitesimal bialgebras. Here the antisymmetric infinitesimal bialgebra structure on $A^{*}$ is $\left(A^{*},-\beta_{A^{*}}\right)$, where $\beta_{A^{*}}: A^{*} \rightarrow A^{*} \otimes A^{*}$ is given in Remark 2.2.4. 
Proof. Let $r \in A \otimes A^{*} \subset\left(A \oplus A^{*}\right) \otimes\left(A \oplus A^{*}\right)$ correspond to the identity map id: $A \rightarrow A$. Let $\left\{e_{1}, \ldots, e_{n}\right\}$ be a basis of $A$ and $\left\{e_{1}^{*}, \ldots, e_{n}^{*}\right\}$ its dual basis. Then $r=\sum_{i=1}^{n} e_{i} \otimes e_{i}^{*}$. Suppose that the associative algebra structure “*” on $A \oplus A^{*}$ is given by $\mathcal{A} D(A)=A \bowtie_{R_{0}^{*}, L_{\circ}^{*}}^{R_{*}^{*}} A^{*}$. Then by Theorem 2.1.4, we have (for any $\left.x, y \in A, a^{*}, b^{*} \in A^{*}\right)$

$$
\begin{aligned}
x * y & =x \cdot y, & a^{*} * b^{*} & =a^{*} \circ b^{*}, \\
x * a^{*} & =R_{.}^{*}(x) a^{*}+L_{\circ}^{*}\left(a^{*}\right) x, & a^{*} * x & =R_{\circ}\left(a^{*}\right) x+L_{.}^{*}(x) a^{*} .
\end{aligned}
$$

If $r$ satisfies eqs. (22) and (23), then

$$
\Delta_{\mathscr{A} D}(u)=(\mathrm{id} \otimes L(u)-R(u) \otimes \mathrm{id}) r
$$

for all $u \in \mathscr{A} \mathscr{D}(A)$ induces an antisymmetric infinitesimal bialgebra structure on $\mathcal{A D}(A)$.

In fact, for eq. (23), we prove a little stronger conclusion (for any $\mu \in \mathcal{A} D(A)$ ):

$(\mathrm{id} \otimes L(\mu)-R(\mu) \otimes \mathrm{id})(r+\sigma(r))$

$$
=\sum_{i}\left(e_{i} \otimes \mu * e_{i}^{*}+e_{i} \otimes \mu * e_{i}-e_{i} * \mu \otimes e_{i}^{*}-e_{i} * \mu \otimes e_{i}\right)=0 .
$$

If $\mu=e_{j}$, then

$$
\begin{aligned}
\sum_{i} e_{i} \otimes e_{j} * e_{i}^{*} & =\sum_{m} e_{m} \cdot e_{j} \otimes e_{m}^{*}+\sum_{i, m}\left\langle e_{i}^{*} \circ e_{m}^{*}, e_{j}\right\rangle e_{i} \otimes e_{m}, \\
\sum_{i} e_{i}^{*} \otimes e_{j} * e_{i} & =\sum_{i} e_{i}^{*} \otimes e_{j} \cdot e_{i}, \\
\sum_{i} e_{i} * e_{j} \otimes e_{i}^{*} & =\sum_{i} e_{i} \cdot e_{j} \otimes e_{i}^{*}, \\
e_{i}^{*} * e_{j} \otimes e_{i} & =\sum_{i, m}\left\langle e_{j}, e_{m}^{*} \circ e_{i}^{*}\right\rangle e_{m} \otimes e_{i}+\sum_{m} e_{m}^{*} \otimes e_{j} \cdot e_{m} .
\end{aligned}
$$

Hence eq. (24) holds for $\mu=e_{j}$ by exchanging some indices. Similarly, eq. (24) holds for $\mu=e_{j}^{*}$. Therefore eq. (23) holds. Furthermore,

$r_{12} r_{13}+r_{13} r_{23}-r_{23} r_{12}=\sum_{i, j}\left\{e_{j} \otimes e_{i} * e_{j}^{*} \otimes e_{i}^{*}-e_{j} \cdot e_{i} \otimes e_{j}^{*} \otimes e_{i}^{*}-e_{i} \otimes e_{j} \otimes e_{i}^{*} \circ e_{j}^{*}\right\}$.

Since $e_{i} * e_{j}^{*}=\sum_{m}\left(\left\langle e_{j}^{*}, e_{m} \cdot e_{i}\right\rangle e_{m}^{*}+\left\langle e_{j}^{*} \circ e_{m}^{*}, e_{i}\right\rangle e_{m}\right)$, it follows that $r_{12} r_{13}+$ $r_{13} r_{23}-r_{23} r_{12}=0$. So $\mathcal{A} \mathscr{D}(A)$ is an antisymmetric infinitesimal bialgebra.

For $e_{i} \in A$, we have

$$
\begin{aligned}
& \Delta_{\mathcal{A} D}\left(e_{i}\right) \\
& \quad=\sum_{m, k}\left\{\left\langle e_{m}^{*}, e_{k} \cdot e_{i}\right\rangle e_{m} \otimes e_{k}^{*}+\left\langle e_{m}^{*} \circ e_{k}^{*}, e_{i}\right\rangle e_{m} \otimes e_{k}-\left\langle e_{m}^{*} \cdot e_{k} \cdot e_{i}\right\rangle e_{m} \otimes e_{k}^{*}\right\} \\
& \quad=\sum_{m, k}\left\langle e_{m}^{*} \circ e_{k}^{*}, e_{i}\right\rangle e_{m} \otimes e_{k}=\Delta_{A}\left(e_{i}\right) .
\end{aligned}
$$


Therefore the inclusion $i_{1}: A \rightarrow A \oplus A^{*}$ is a homomorphism of antisymmetric infinitesimal bialgebras. Similarly, the inclusion $i_{2}: A^{*} \rightarrow A \oplus A^{*}$ is also a homomorphism of antisymmetric infinitesimal bialgebras since $\Delta_{\mathcal{A D}}\left(e_{i}^{*}\right)=-\beta_{A^{*}}\left(e_{i}^{*}\right)$, where $\beta_{A^{*}}$ is given in Remark 2.2.4.

Definition 2.3.7. Let $\left(A, A^{*}\right)$ be an antisymmetric infinitesimal bialgebra. With the antisymmetric infinitesimal bialgebra structure given in Theorem 2.3.6, $A \oplus A^{*}$ is called an associative double of $A$. We denote it by $\mathcal{A D}(A)$.

Remark 2.3.8. If we use the opposite algebra, then Theorem 2.3.6 and its proof overlap [A3], Theorem 5.9 and Proposition 5.10 partly. Moreover, the associative double $\mathcal{A D}(A)$ is a balanced Drinfeld double which was denoted by $D_{b}(A)$ in [A3].

Corollary 2.3.9. Let $\left(A, A^{*}\right)$ be an antisymmetric infinitesimal bialgebra. Then the associative double $\mathcal{A D}(A)$ of $A$ is an antisymmetric infinitesimal bialgebra and it is a symmetric Frobenius algebra with the bilinear form given by eq. (2).

\subsection{The associative Yang-Baxter equation and its properties}

Corollary 2.4.1. Let $A$ be an associative algebra and $r \in A \otimes A$. Suppose that $r$ is antisymmetric. Then the map $\Delta$ defined by eq. (21) induces an associative algebra structure on $A^{*}$ such that $\left(A, A^{*}\right)$ is an antisymmetric infinitesimal bialgebra if

$$
r_{12} r_{13}+r_{13} r_{23}-r_{23} r_{12}=0 \text {. }
$$

Definition 2.4.2. Let $A$ be an associative algebra and $r \in A \otimes A$. Eq. (25) is called associative Yang-Baxter equation in $A$.

Remark 2.4.3. In [A1] and [A3], the associative Yang-Baxter equation is given as

$$
r_{13} r_{12}+r_{23} r_{13}-r_{12} r_{23}=0 \text {. }
$$

Note that eq. (25) is eq. (26) in the opposite algebra. Moreover, if $r$ satisfies $(L(x) \otimes \mathrm{id} \otimes \mathrm{id}-\mathrm{id} \otimes \mathrm{id} \otimes R(x))\left(r_{12}+r_{21}\right)=0$, then ([A3], Lemma 3.4)

$$
\sigma_{13}\left(r_{12} r_{13}+r_{13} r_{23}-r_{23} r_{12}\right)=r_{13} r_{12}+r_{23} r_{13}-r_{12} r_{23},
$$

where the linear map $\sigma_{13}: A \otimes A \otimes A \rightarrow A \otimes A \otimes A$ is given by $\sigma_{13}(x \otimes y \otimes z)=$ $z \otimes y \otimes x$ for any $x, y, z \in A$. In particular, when $r$ is antisymmetric, the above two associative Yang-Baxter equations are equivalent.

In order to be self-contained, in the following we give some properties of the associative Yang-Baxter equation from the point of view of Frobenius algebras, although some of them have already been given in [A3]. Let $A$ be a vector space. For any $r \in A \otimes A, r$ can be regarded as a map from $A^{*}$ to $A$ in the following way:

$$
\left\langle u^{*} \otimes v^{*}, r\right\rangle=\left\langle u^{*}, r\left(v^{*}\right)\right\rangle \text { for all } u^{*}, v^{*} \in A^{*} .
$$


Proposition 2.4.4. Let $(A, \cdot)$ be an associative algebra and let $r \in A \otimes A$ be an antisymmetric solution of the associative Yang-Baxter equation in $A$. Then the associative algebra structure on the associative double $\mathcal{A D}(A)$ is given from the products in $A$ as follows:

$$
\begin{aligned}
a^{*} * b^{*} & =a^{*} \circ b^{*}=R_{.}^{*}\left(r\left(a^{*}\right)\right) b^{*}+L_{.}^{*}\left(r\left(b^{*}\right)\right) a^{*} \quad \text { for any } a^{*}, b^{*} \in A^{*}, \\
x * a^{*} & =x \cdot r\left(a^{*}\right)-r\left(R_{.}^{*}(x) a^{*}\right)+R_{.}^{*}(x) a^{*} \quad \text { for any } x \in A, a^{*} \in A^{*}, \\
a^{*} * x & =r\left(a^{*}\right) \cdot x-r\left(L_{.}^{*}(x) a^{*}\right)+L_{.}^{*}(x) a^{*} \quad \text { for any } x \in A, a^{*} \in A^{*} .
\end{aligned}
$$

Proof. Let $\left\{e_{1}, \ldots, e_{n}\right\}$ be a basis of $A$ and $\left\{e_{1}^{*}, \ldots, e_{n}^{*}\right\}$ its dual basis. Suppose that $e_{i} \cdot e_{j}=\sum_{k} c_{i j}^{k} e_{k}$ and $r=\sum_{i, j} a_{i j} e_{i} \otimes e_{j}$, where $a_{i j}=-a_{j i}$. Then for any $i$, we have

$$
\Delta\left(e_{i}\right)=\sum_{\alpha, \beta, l} a_{\alpha \beta}\left(c_{i \beta}^{l} e_{\alpha} \otimes e_{l}-c_{\alpha i}^{l} e_{l} \otimes e_{\beta}\right)=\sum_{\alpha, \beta} \sum_{l}\left(a_{\alpha l} c_{i l}^{\beta}-a_{l \beta} c_{l i}^{\alpha}\right) e_{\alpha} \otimes e_{\beta} .
$$

Therefore we have (for any $i, j$ )

$$
\begin{aligned}
e_{i}^{*} \circ e_{j}^{*} & =\sum_{l, t}\left(a_{i l} c_{t l}^{j}-a_{l j} c_{l t}^{i}\right) e_{t}^{*} \\
& =\sum_{l, t}\left(a_{i l}\left\langle e_{t} \cdot e_{l}, e_{j}^{*}\right\rangle-a_{l j}\left\langle e_{l} \cdot e_{t}, e_{i}^{*}\right\rangle\right) e_{t}^{*} \\
& =\sum_{t}\left(\left\langle e_{t} \cdot r\left(e_{i}^{*}\right), e_{j}^{*}\right\rangle+\left\langle r\left(e_{j}^{*}\right) \cdot e_{t}, e_{i}^{*}\right\rangle\right) e_{t}^{*} \\
& =R_{.}^{*}\left(r\left(e_{i}^{*}\right)\right) e_{j}^{*}+L_{.}^{*}\left(r\left(e_{j}^{*}\right)\right) e_{i}^{*} .
\end{aligned}
$$

Similarly, eqs. (28) and (29) hold.

Theorem 2.4.5 ([A3], Proposition 2.1). Let $A$ be an associative algebra and $r \in$ $A \otimes A$. Suppose that $r$ is antisymmetric and non-degenerate. Then $r$ is a solution of the associative Yang-Baxter equation in $A$ if and only if the inverse of the isomorphism $A^{*} \rightarrow A$ induced by $r$, regarded as a bilinear form $\omega$ on $A$ (that is, $\omega(x, y)=$ $\left\langle r^{-1} x, y\right\rangle$ for any $\left.x, y \in A\right)$, is a Connes cocycle.

Corollary 2.4.6. Let $(A, \cdot)$ be an associative algebra and let $r \in A \otimes A$ be a nondegenerate antisymmetric solution of the associative Yang-Baxter equation in A. Suppose the associative algebra structure "०" on $A^{*}$ is induced by $r$ from eq. (27). Then we have

$$
a^{*} \circ b^{*}=r^{-1}\left(r\left(a^{*}\right) \cdot r\left(b^{*}\right)\right) \text { for all } a^{*}, b^{*} \in A^{*} .
$$

Therefore $r: A^{*} \rightarrow A$ is an isomorphism of associative algebras. 
Proof. Set $\omega(x, y)=\left\langle r^{-1}(x), y\right\rangle$ for any $x, y \in A$. Then $\omega$ is a Connes cocycle of $A$. Hence

$$
\begin{aligned}
\left\langle a^{*} \circ b^{*}, x\right\rangle & =\left\langle r\left(b^{*}\right) \cdot x, a^{*}\right\rangle+\left\langle x \cdot r\left(a^{*}\right), b^{*}\right\rangle \\
& =\omega\left(r\left(a^{*}\right), r\left(b^{*}\right) \cdot x\right)+\omega\left(r\left(b^{*}\right), x \cdot r\left(a^{*}\right)\right) \\
& =-\omega\left(x, r\left(a^{*}\right) \cdot r\left(b^{*}\right)\right) \\
& =\left\langle r^{-1}\left(r\left(a^{*}\right) \cdot r\left(b^{*}\right)\right), x\right\rangle
\end{aligned}
$$

for all $a^{*}, b^{*} \in A^{*}, x \in A$. So eq. (30) holds. Therefore $r$ is an isomorphism of associative algebras.

Next we turn to the general antisymmetric solutions of associative Yang-Baxter equation.

Theorem 2.4.7. Let $(A, \cdot)$ be an associative algebra and $r \in A \otimes A$ antisymmetric. Then $r$ is a solution of the associative Yang-Baxter equation in $A$ if and only if $r$ satisfies

$$
r\left(a^{*}\right) \cdot r\left(b^{*}\right)=r\left(R_{.}^{*}\left(r\left(a^{*}\right)\right) b^{*}+L_{.}^{*}\left(r\left(b^{*}\right)\right) a^{*}\right)
$$

for all $a^{*}, b^{*} \in A^{*}$.

Proof. Let $\left\{e_{1}, \ldots, e_{n}\right\}$ be a basis of $A$ and $\left\{e_{1}^{*}, \ldots, e_{n}^{*}\right\}$ its dual basis. Suppose that $e_{i} \cdot e_{j}=\sum_{k} c_{i j}^{k} e_{k}$ and $r=\sum_{i, j} a_{i j} e_{i} \otimes e_{j}, a_{i j}=-a_{j i}$. Hence $r\left(e_{i}^{*}\right)=\sum_{k} a_{k i} e_{k}$. Then $r$ is a solution of the associative Yang-Baxter equation in $A$ if and only if (for any $i, j, k)$

$$
\sum_{m, l}\left\{c_{k l}^{m} a_{i k} a_{j l}-c_{l k}^{i} a_{j l} a_{k m}-c_{l k}^{j} a_{l m} a_{i k}\right\}=0 .
$$

The left-hand side of the above equation is just the coefficient of $e_{m}$ in

$$
r\left(e_{i}^{*}\right) \cdot r\left(e_{j}^{*}\right)-r\left(R_{.}^{*}\left(r\left(e_{i}^{*}\right)\right) e_{j}^{*}+L_{.}^{*}\left(r\left(e_{j}^{*}\right)\right) e_{i}^{*}\right) .
$$

Therefore the conclusion follows.

Combining Proposition 2.4.4 and Theorem 2.4.7, we have the following conclusion which extends Corollary 2.4.6.

Corollary 2.4.8. Let $(A, \cdot)$ be an associative algebra and let $r \in A \otimes A$ be an antisymmetric solution of the associative Yang-Baxter equation in A. Suppose the associative algebra structure "०” on $A^{*}$ is induced by $r$ from eq. (27). Then we have

$$
r\left(a^{*} \circ b^{*}\right)=r\left(a^{*}\right) \cdot r\left(b^{*}\right) \text { for all } a^{*}, b^{*} \in A^{*} .
$$

Therefore $r: A^{*} \rightarrow A$ is an homomorphism of associative algebras. 
Recall that two Frobenius algebras $\left(A_{1}, \mathscr{B}_{1}\right)$ and $\left(A_{2}, \mathscr{B}_{2}\right)$ are isomorphic if and only if there exists an isomorphism of associative algebras $\varphi: A_{1} \rightarrow A_{2}$ such that

$$
\mathcal{B}_{1}(x, y)=\varphi^{*} \mathscr{B}_{2}(x, y)=\mathscr{B}_{2}(\varphi(x), \varphi(y))
$$

for all $x, y \in A_{1}$.

Theorem 2.4.9. Let $(A, \cdot)$ be an associative algebra. Then, as a Frobenius algebra, the Frobenius algebra $\left(A \bowtie_{R_{\circ}^{*}, L_{\circ}^{*}}^{R_{*}^{*}, L^{*}} A^{*}, \mathcal{B}\right)$ given by an antisymmetric solution $r$ of the associative Yang-Baxter equation in $A$ is isomorphic to the Frobenius algebra $\left(A \ltimes R_{*}^{*}, L^{*} A^{*}, \mathcal{B}\right)$, where $\mathcal{B}$ is given by eq. (2). However, in general, they are not isomorphic as the double constructions of Frobenius algebras (or equivalently, as antisymmetric infinitesimal bialgebras).

Proof. Let $r$ be an antisymmetric solution of associative the Yang-Baxter equation in $A$. Define a linear map $\varphi: A \ltimes_{R^{*}, L^{*}} A^{*} \rightarrow A \bowtie_{R_{\circ}^{*}, L_{\circ}^{*}}^{R^{*}, L^{*}} A^{*}$ satisfying

$$
\varphi(x)=x, \quad \varphi\left(a^{*}\right)=-r\left(a^{*}\right)+a^{*}
$$

for all $x \in A, a^{*} \in A^{*}$. It is straightforward to show that $\varphi$ is an isomorphism of associative algebras. Moreover,

$$
\begin{aligned}
\varphi^{*} \mathcal{B}\left(x+a^{*}, y+b^{*}\right) & =\left\langle a^{*},-r\left(b^{*}\right)+y\right\rangle+\left\langle x-r\left(a^{*}\right), b^{*}\right\rangle \\
& =\left\langle a^{*}, y\right\rangle+\left\langle x, b^{*}\right\rangle \\
& =\mathscr{B}\left(x+a^{*}, y+b^{*}\right) .
\end{aligned}
$$

Therefore $\varphi$ is an isomorphism of Frobenius algebras. However in general, as antisymmetric infinitesimal bialgebras, they are not isomorphic. In fact, if $\psi$ is an isomorphism of antisymmetric infinitesimal bialgebras between $A \ltimes R^{*}, L^{*} A^{*}$ and $A \bowtie_{R_{\circ}^{*}, L_{\circ}^{*}}^{R_{*}^{*}, L^{*}} A^{*}$, then for any $u^{*}, v^{*} \in A^{*}$ there exist $a^{*}, b^{*} \in A^{*}$ such that $\psi\left(a^{*}\right)=u^{*}, \psi\left(b^{*}\right)=v^{*}$. However, $\psi\left(a^{*} \circ b^{*}\right)=0$ and $\psi\left(a^{*}\right) * \psi\left(b^{*}\right)=$ $u^{*} * v^{*}=R^{*}\left(r\left(a^{*}\right)\right) b^{*}+L^{*}\left(r\left(b^{*}\right)\right) a^{*}$ is not zero in general, which is a contradiction.

Corollary 2.4.10. Let $(A, \cdot)$ be an associative algebra. Then as Frobenius algebras, the Frobenius algebras $\left(A \bowtie_{R_{0}^{*}, L_{\circ}^{*}}^{R_{*}^{*}, L^{*}} A^{*}, \mathcal{B}\right)$ given by all antisymmetric solutions of the associative Yang-Baxter equation in A are isomorphic to the Frobenius algebra $\left(A \ltimes_{R^{*}, L^{*}} A^{*}, \mathcal{B}\right)$ given by the zero solution.

\subsection{The associative Yang-Baxter equation and $\mathcal{O}$-operators}

Definition 2.5.1. Let $(A, \cdot)$ be an associative algebra and $(l, r, V)$ a bimodule. A linear map $T: V \rightarrow A$ is called an $\mathcal{O}$-operator associated to $(l, r, V)$ if $T$ satisfies

$$
T(u) \cdot T(v)=T(l(T(u)) v+r(T(v) u)) \quad \text { for all } u, v \in V .
$$


Example 2.5.2. Let $(A, \cdot)$ be an associative algebra. Then the identity map id is an $\mathcal{O}$-operator associated to the bimodule $(L, 0)$ or $(0, R)$.

Example 2.5.3. Let $(A, \cdot)$ be an associative algebra. A linear map $R: A \rightarrow A$ is called a Rota-Baxter operator on $A$ of weight zero ([Bax], [Rot]) if $R$ satisfies

$$
R(x) \cdot R(y)=R(R(x) \cdot y+x \cdot R(y)) \text { for all } x, y \in A \text {. }
$$

In fact, a Rota-Baxter operator on $A$ is just an $\mathcal{O}$-operator associated to the bimodule $(L, R)$.

Example 2.5.4. Let $(A, \cdot)$ be an associative algebra and $r \in A \otimes A$ antisymmetric. Then $r$ is a solution of associative Yang-Baxter equation in $A$ if and only if $r$ is an $\mathcal{O}$-operator associated to the bimodule $\left(R^{*}, L^{*}\right)$.

Theorem 2.5.5 ([BGN1]). Let $(A, \cdot)$ be an associative algebra and $(l, r, V)$ a bimodule. Let $\left(r^{*}, l^{*}, V^{*}\right)$ be the bimodule of $A$ given by Lemma 2.1.2. Let $T: V \rightarrow A$ be a linear map which is identified as an element in $\left(A \ltimes_{r^{*}, l^{*}} V^{*}\right) \otimes\left(A \ltimes_{r^{*}, l^{*}} V^{*}\right)$. Then $r=T-\sigma(T)$ is an antisymmetric solution of the associative Yang-Baxter equation in $A \ltimes_{r^{*}, l^{*}} V^{*}$ if and only if $T$ is an $\mathcal{O}$-operator associated to the bimodule $(l, r, V)$.

Corollary 2.5.6 (Cf. Corollary 3.1.5). Let $(A, \cdot)$ be an associative algebra. Then

$$
r=\sum_{i}^{n}\left(e_{i} \otimes e_{i}^{*}-e_{i}^{*} \otimes e_{i}\right)
$$

is a solution of the associative Yang-Baxter equation in $A \ltimes_{R^{*}, 0} A^{*}$ or $A \ltimes_{0, L^{*}} A^{*}$, where $\left\{e_{1}, \ldots, e_{n}\right\}$ is a basis of $A$ and $\left\{e_{1}^{*}, \ldots, e_{n}^{*}\right\}$ is its dual basis. Moreover there is a natural Connes cocycle $\omega$ on $A \ltimes_{R^{*}, 0} A^{*}$ or $A \ltimes_{0, L^{*}}$ induced by $r^{-1}: A \oplus A^{*} \rightarrow$ $\left(A \oplus A^{*}\right)^{*}$, which is given by eq. (7).

Proof. Note that id is an $\mathcal{O}$-operator associated to the bimodule $(L, 0, A)$ or $(0, R, A)$. Then the conclusion follows from Theorems 2.5.5 and 2.4.5.

\section{Dendriform algebras}

3.1. $\mathcal{O}$-operators and dendriform algebras. There are close relations between $\mathcal{O}$-operators and a class of algebras, namely, dendriform algebras, which are given in [BGN2]. In order to be self-contained, we list them in this subsection.

Definition 3.1.1 ([Lo1]). Let $A$ be a vector space over a field $\mathbb{F}$ with two bilinear products denoted by $\prec$ and $\succ$. Then $(A, \prec, \succ)$ is called a dendriform algebra if, for 
any $x, y, z \in A$,

$$
\begin{aligned}
& (x \prec y) \prec z=x \prec(y * z), \\
& (x \succ y) \prec z=x \succ(y \prec z), \\
& x \succ(y \succ z)=(x * y) \succ z,
\end{aligned}
$$

where $x * y=x \prec y+x \succ y$.

Let $(A, \prec, \succ)$ be a dendriform algebra. For any $x \in A$, let $L_{\succ}(x), R_{\succ}(x)$ and $L_{\prec}(x), R_{\prec}(x)$ denote the left and right multiplication operators of $(A, \prec)$ and $(A, \succ)$, respectively, that is,

$$
L_{\succ}(x)(y)=x \succ y, R_{\succ}(x) y=y \succ x, L_{\prec}(x) y=x \prec y, R_{\prec}(x)(y)=y \prec x
$$

for all $x, y \in A$. Moreover, let $L_{\succ}, R_{\succ}, L_{\prec}, R_{\prec}: A \rightarrow \mathfrak{g l}(A)$ be four linear maps with $x \rightarrow L_{\succ}(x), x \rightarrow R_{\succ}(x), x \rightarrow L_{\prec}(x)$ and $x \rightarrow R_{\prec}(x)$, respectively. It is known that the product given by ([Lo1])

$$
x * y=x \prec y+x \succ y, \quad \text { for all } x, y \in A,
$$

defines an associative algebra. We call $(A, *)$ the associated associative algebra of $(A, \succ, \prec)$ and $(A, \succ, \prec)$ is called a compatible dendriform algebra structure on the associative algebra $(A, *)$. Moreover, $\left(L_{\succ}, R_{\prec}\right)$ is a bimodule of the associated associative algebra $(A, *)$.

Theorem 3.1.2 ([BGN2]). Let $A$ be an associative algebra and $(l, r, V)$ a bimodule. Let $T: V \rightarrow A$ be an $\mathcal{O}$-operator associated to $(l, r, V)$. Then there exists a dendriform algebra structure on $V$ given by

$$
u \succ v=l(T(u)) v, \quad u \prec v=r(T(v)) u
$$

for all $u, v \in V$. So there is an associated associative algebra structure on $V$ given by eq. (32) and $T$ is a homomorphism of associative algebras. Moreover, $T(V)=\{T(v) \mid v \in V\} \subset A$ is an associative subalgebra of $A$ and there is an induced dendriform algebra structure on $T(V)$ given by

$$
T(u) \succ T(v)=T(u \succ v), \quad T(u) \prec T(v)=T(u \prec v)
$$

for all $u, v \in V$. Its corresponding associated associative algebra structure on $T(V)$ given by eq. (32) is just the associative subalgebra structure of $A$ and $T$ is a homomorphism of dendriform algebras.

Corollary 3.1.3 ([BGN2]). Let $(A, *)$ be an associative algebra. There is a compatible dendriform algebra structure on $A$ if and only if there exists an invertible $\mathcal{O}$-operator of $(A, *)$. 
In fact, if $T$ is an invertible $\mathcal{O}$-operator associated to a bimodule $(l, r, V)$, then the compatible dendriform algebra structure on $A$ is given by

$$
x \succ y=T\left(l(x) T^{-1}(y)\right), \quad x \prec y=T\left(r(y) T^{-1}(x)\right)
$$

for all $x, y \in A$. Conversely, let $(A, \succ, \prec)$ be a dendriform algebra and $(A, *)$ the associated associative algebra. Then the identity map id is an $\mathcal{O}$-operator associated to the bimodule $\left(L_{\succ}, R_{\prec}\right)$ of $(A, *)$.

Remark 3.1.4. If $T$ is an invertible $\mathcal{O}$-operator associated to a bimodule $(l, r, V)$, then the linear map $f=T^{-1}: A \rightarrow V$ satisfies

$$
f(x * y)=l(x) f(y)+r(y) f(x) \quad \text { for all } x, y \in A .
$$

Such a linear map is a 1-cocycle of $(A, *)$ associated to the bimodule $(l, r, V)$.

Corollary 3.1.5 ([BGN2]). Let $(A, \succ, \prec)$ be a dendriform algebra. Then

$$
r=\sum_{i}^{n}\left(e_{i} \otimes e_{i}^{*}-e_{i}^{*} \otimes e_{i}\right)
$$

is a solution of the associative Yang-Baxter equation in $A \ltimes_{R_{*}^{*}, L_{>}^{*}} A^{*}$, where $\left\{e_{1}, \ldots, e_{n}\right\}$ is a basis of $A$ and $\left\{e_{1}^{*}, \ldots, e_{n}^{*}\right\}$ is its dual basis. Moreover there is a natural Connes cocycle $\omega$ on $A \ltimes_{R_{\prec}^{*}, L_{\succ}^{*}} A^{*}$ induced by $r^{-1}: A \oplus A^{*} \rightarrow\left(A \oplus A^{*}\right)^{*}$, which is given by eq. (7).

Remark 3.1.6. It is easy to see that Corollary 2.5 .6 is a special case of the above conclusion, that is, the former corresponds to the trivial dendriform algebra structure on an associative algebra $(A, \cdot)$ given by $\succ=\cdot, \prec=0$ or $\succ=0, \prec=\bullet$.

\subsection{Bimodules and matched pairs of dendriform algebras}

Definition 3.2.1 ([A4]). Let $(A, \succ, \prec)$ be a dendriform algebra and $V$ a vector space. Let $l_{\succ}, r_{\succ}, l_{<}, r_{\prec}: A \rightarrow \operatorname{gl}(V)$ be four linear maps. Then $V$ (or $\left(l_{\succ}, r_{\succ}, l_{<}, r_{<}\right)$, or $\left.\left(l_{\succ}, r_{\succ}, l_{<}, r_{<}, V\right)\right)$ is called a bimodule of $A$ if the following equations hold for any $x, y \in A$ :

$$
\begin{aligned}
& l_{\prec}(x \prec y)=l_{\prec}(x) l_{*}(y), r_{\prec}(x) l_{\prec}(y)=l_{\prec}(y) r_{*}(x), r_{\prec}(x) r_{\prec}(y)=r_{\prec}(y * x), \\
& l_{\prec}(x \succ y)=l_{\succ}(x) l_{\prec}(y), r_{\prec}(x) l_{\succ}(y)=l_{\succ}(y) r_{\prec}(x), r_{\prec}(x) r_{\succ}(y)=r_{\succ}(y \prec x), \\
& l_{\succ}(x * y)=l_{\succ}(x) l_{\succ}(y), r_{\succ}(x) l_{*}(y)=l_{\succ}(y) r_{\succ}(x), r_{\succ}(x) r_{*}(y)=r_{\succ}(y \succ x) \text {, } \\
& \text { where } x * y=x \succ y+x \prec y, l_{*}=l_{\succ}+l_{\prec}, r_{*}=r_{\succ}+r_{\prec} \text {. }
\end{aligned}
$$

By a direct computation or according to [Sc], $\left(l_{\succ}, r_{\succ}, l_{\prec}, r_{\prec}, V\right)$ is a bimodule of a dendriform algebra $(A, \succ, \prec)$ if and only if there exists a dendriform algebra 
structure on the direct sum $A \oplus V$ of the underlying vector spaces of $A$ and $V$ given by

$$
\begin{aligned}
& (x+u) \succ(y+v)=x \succ y+l_{\succ}(x) v+r_{\succ}(y) u, \\
& (x+u) \prec(y+v)=x \prec y+l_{\prec}(x) v+r_{\prec}(y) u
\end{aligned}
$$

for all $x, y \in A, u, v \in V$. We denote it by $A \ltimes_{l_{\succ}, r_{\succ}, l_{<}, r_{\prec}} V$.

Proposition 3.2.2. Let $\left(l_{\succ}, r_{\succ}, l_{\prec}, r_{\prec}, V\right)$ be a bimodule of a dendriform algebra $(A, \succ, \prec)$. Let $(A, *)$ be the associated associative algebra. Then we have the following results.

(1) Both $\left(l_{\succ}, r_{\prec}, V\right)$ and $\left(l_{\succ}+l_{<}, r_{\succ}+r_{<}, V\right)$ are bimodules of $(A, *)$.

(2) For any bimodule $(l, r, V)$ of $(A, *),(l, 0,0, r, V)$ is a bimodule of $(A, \succ, \prec)$.

(3) Both $\left(l_{\succ}+l_{\prec}, 0,0, r_{\succ}+r_{\prec}, V\right)$ and $\left(l_{\succ}, 0,0, r_{\prec}, V\right)$ are bimodules of $(A, \succ, \prec)$.

(4) The dendriform algebras $A \ltimes_{l_{\succ}, r_{\succ}, l_{<}, r_{\prec}} V$ and $A \ltimes_{l_{\succ}+l_{<}, 0,0, r_{\succ}+r_{\prec}} V$ have the same associated associative algebra $A \ltimes_{l_{\succ}+l_{<,}, r_{\succ}+r_{\prec}} V$.

(5) Let $l_{\succ}^{*}, r_{\succ}^{*}, l_{\prec}^{*}, r_{\prec}^{*}: A \rightarrow \mathfrak{g l}\left(V^{*}\right)$ be the linear maps given by

$$
\begin{array}{ll}
\left\langle l_{\succ}^{*}(x) a^{*}, y\right\rangle=\left\langle l_{\succ}(x) y, a^{*}\right\rangle, & \left\langle r_{\succ}^{*}(x) a^{*}, y\right\rangle=\left\langle r_{\succ}(x) y, a^{*}\right\rangle, \\
\left\langle l_{\prec}^{*}(x) a^{*}, y\right\rangle=\left\langle l_{\prec}(x) y, a^{*}\right\rangle, & \left\langle r_{\prec}^{*}(x) a^{*}, y\right\rangle=\left\langle r_{\prec}(x) y, a^{*}\right\rangle .
\end{array}
$$

Then $\left(r_{\succ}^{*}+r_{\prec}^{*},-l_{\prec}^{*},-r_{\succ}^{*}, l_{\succ}^{*}+l_{\prec}^{*}, V^{*}\right)$ is a bimodule of $(A, \succ, \prec)$.

(6) Both $\left(r_{\succ}^{*}+r_{\prec}^{*}, 0,0, l_{\succ}^{*}+l_{<}^{*}, V^{*}\right)$ and $\left(r_{<}^{*}, 0,0, l_{\succ}^{*}, V^{*}\right)$ are bimodules of $(A, \succ, \prec)$.

(7) Both $\left(r_{\succ}^{*}+r_{\prec}^{*}, l_{\succ}^{*}+l_{\prec}^{*}, V^{*}\right)$ and $\left(r_{\prec}^{*}, l_{\succ}^{*}, V^{*}\right)$ are bimodules of $(A, *)$.

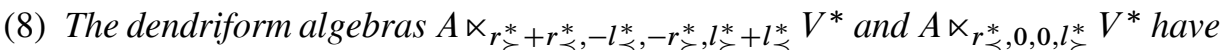
the same associative algebra $A \ltimes_{r_{*}^{*}, l_{\gtrless}^{*}} V^{*}$.

Proof. This is straightforward.

Example 3.2.3. Let $(A, \succ, \prec)$ be a dendriform algebra. Then

$$
\left(L_{\succ}, R_{\succ}, L_{\prec}, R_{\prec}, A\right), \quad\left(L_{\succ}, 0,0, R_{\prec}, A\right), \quad\left(L_{\succ}+L_{\prec}, 0,0, R_{\succ}+R_{\prec}, A\right)
$$

are bimodules of $(A, \prec, \succ)$. On the other hand,

$$
\begin{gathered}
\left(R_{\succ}^{*}+R_{\prec}^{*},-L_{\prec}^{*},-R_{\succ}^{*}, L_{\succ}^{*}+L_{\prec}^{*}, A^{*}\right), \quad\left(R_{\prec}^{*}, 0,0, L_{\succ}^{*}, A^{*}\right), \\
\left(R_{\succ}^{*}+R_{\prec}^{*}, 0,0, L_{\succ}^{*}+L_{\prec}^{*}, A^{*}\right)
\end{gathered}
$$

are bimodules of $(A, \succ, \prec)$, too. There are two compatible dendriform algebra structures,

$$
A \ltimes_{R_{\succ}^{*}+R_{\prec}^{*},-L_{\prec}^{*},-R_{\succ}^{*}, L_{\succ}^{*}+L_{\prec}^{*}} A^{*} \quad \text { and } \quad A \ltimes_{R_{\prec}^{*}, 0,0, L_{\succ}^{*}} A^{*},
$$

on the same associative algebra $A \ltimes_{R_{\prec}^{*}, L_{\succ}^{*}} A^{*}$. 
Theorem 3.2.4. Let $\left(A, \succ_{A}, \prec_{A}\right)$ and $\left(B, \succ_{B}, \prec_{B}\right)$ be two dendriform algebras. Suppose that there are linear maps $l_{\succ_{A}}, r_{\succ_{A}}, l_{\succ_{A}}, r_{\succ_{A}}: A \rightarrow \operatorname{gl}(B)$ and $l_{\succ_{B}}$, $r_{\succ_{B}}, l_{\prec_{B}}, r_{\prec_{B}}: B \rightarrow \operatorname{gl}(A)$ such that $\left(l_{\succ_{A}}, r_{\succ_{A}}, l_{\prec_{A}}, r_{\prec_{A}}\right)$ is a bimodule of $A$ and $\left(l_{\succ_{B}}, r_{\succ_{B}}, l_{\prec_{B}}, r_{\prec_{B}}\right)$ is a bimodule of $B$ and they satisfy the following 18 equations:

$$
\begin{aligned}
& r_{\prec_{A}}(x)\left(a \prec_{B} b\right)=a \prec_{B}\left(r_{A}(x) b\right)+r_{\prec_{A}}\left(l_{B}(b) x\right) a, \\
& l_{\prec_{A}}\left(l_{\prec_{B}}(a) x\right) b+\left(r_{\prec_{A}}(x) a\right) \prec_{B} b=a \prec_{B}\left(l_{A}(x) b\right)+r_{\prec_{A}}\left(r_{B}(b) x\right) a, \\
& l_{\prec_{A}}(x)\left(a *_{B} b\right)=\left(l_{\prec_{A}}(x) a\right) \prec_{B} b+l_{\prec_{A}}\left(r_{\prec_{B}}(a) x\right) b, \\
& r_{\prec_{A}}(x)\left(a \succ_{B} b\right)=r_{\succ_{A}}\left(l_{\prec_{B}}(b) x\right) a+a \succ_{B}\left(r_{\prec_{A}}(x) b\right), \\
& l_{\prec_{A}}\left(l_{\succ_{B}}(a) x\right) b+\left(r_{\succ_{A}}(x) a\right) \prec_{B} b=a \succ_{B}\left(l_{\prec_{A}}(x) b\right)+r_{\succ_{A}}\left(r_{\prec_{B}}(b) x\right) a \text {, } \\
& l_{\succ_{A}}(x)\left(a \prec_{B} b\right)=\left(l_{\succ_{A}}(x) a\right) \prec_{B} b+l_{\prec_{A}}\left(r_{\succ_{B}}(a) x\right) b, \\
& r_{\succ_{A}}(x)\left(a *_{B} b\right)=a \succ_{B}\left(r_{\succ_{A}}(x) b\right)+r_{\succ_{A}}\left(l_{\succ_{B}}(b) x\right) a, \\
& a \succ_{B}\left(l_{\succ_{A}}(x) b\right)+r_{\succ_{A}}\left(r_{\succ_{B}}(b) x\right) a=l_{\succ_{A}}\left(l_{B}(a) x\right) b+\left(r_{A}(x) a\right) \succ_{B} b \text {, } \\
& l_{\succ_{A}}(x)\left(a \succ_{B} b\right)=\left(l_{A}(x) a\right) \succ_{B} b+l_{\succ_{A}}\left(r_{B}(a) x\right) b \text {, } \\
& r_{\prec_{B}}(a)\left(x \prec_{A} y\right)=x \prec_{A}\left(r_{B}(a) y\right)+r_{\prec_{B}}\left(l_{A}(y) a\right) x, \\
& l_{\prec_{B}}\left(l_{\prec_{A}}(x) a\right) y+\left(r_{\prec_{B}}(a) x\right) \prec_{A} y=x \prec_{A}\left(l_{B}(a) y\right)+r_{\prec_{B}}\left(r_{A}(y) a\right) x \text {, } \\
& l_{\prec_{B}}(a)\left(x *_{A} y\right)=\left(l_{\prec_{B}}(a) x\right) \prec_{A} y+l_{\prec_{B}}\left(r_{\prec_{A}}(x) a\right) y, \\
& r_{\prec_{B}}(a)\left(x \succ_{A} y\right)=r_{\succ_{B}}\left(l_{\prec_{A}}(y) a\right) x+x \succ_{A}\left(r_{\prec_{B}}(a) y\right), \\
& l_{\prec_{B}}\left(l_{\succ_{A}}(x) a\right) y+\left(r_{\succ_{B}}(a) x\right) \prec_{A} y=x \succ_{A}\left(l_{\prec_{B}}(a) y\right)+r_{\succ_{B}}\left(r_{\prec_{A}}(y) a\right) x \text {, } \\
& l_{\succ_{B}}(a)\left(x \prec_{A} y\right)=\left(l_{\succ_{B}}(a) x\right) \prec_{A} y+l_{\prec_{B}}\left(r_{\succ_{A}}(x) a\right) y, \\
& r_{\succ_{B}}(a)\left(x *_{A} y\right)=x \succ_{A}\left(r_{\succ_{B}}(a) y\right)+r_{\succ_{B}}\left(l_{\succ_{A}}(y) a\right) x \text {, } \\
& x \succ_{A}\left(l_{\succ_{B}}(a) y\right)+r_{\succ_{B}}\left(r_{\succ_{A}}(y) a\right) x=l_{\succ_{B}}\left(l_{A}(x) a\right) y+\left(r_{B}(a) x\right) \succ_{A} y \text {, } \\
& l_{\succ_{B}}(a)\left(x \succ_{A} y\right)=\left(l_{B}(a) x\right) \succ_{A} y+l_{\succ_{B}}\left(r_{A}(x) a\right) y
\end{aligned}
$$

for any $x, y \in A, a, b \in B$ and $l_{A}=l_{\succ_{A}}+l_{\prec_{A}}, r_{A}=r_{\succ_{A}}+r_{\prec_{A}}, l_{B}=l_{\succ_{B}}+l_{\prec_{B}}$, $r_{B}=r_{\succ_{B}}+r_{\prec_{B}}$. Then there is a dendriform algebra structure on the direct sum $A \oplus B$ of the underlying vector spaces of $A$ and $B$ given by

$$
\begin{aligned}
& (x+a) \succ(y+b)=\left(x \succ_{A} y+r_{\succ_{B}}(b) x+l_{\succ_{B}}(a) y\right) \\
& +\left(l_{\succ_{A}}(x) b+r_{\succ_{A}}(y) a+a \succ_{B} b\right), \\
& (x+a) \prec(y+b)=\left(x \prec_{A} y+r_{\prec_{B}}(b) x+l_{\prec_{B}}(a) y\right) \\
& \left.+\left(l_{\prec_{A}} x\right) b+r_{\prec_{A}}(y) a+a \prec_{B} b\right)
\end{aligned}
$$

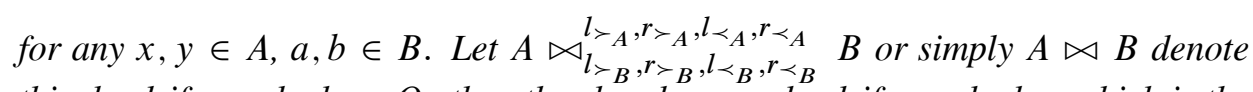
this dendriform algebra. On the other hand, every dendriform algebra which is the direct sum of the underlying vector spaces of two subalgebras can be obtained in this way.

Proof. This is straightforward. 
Definition 3.2.5. Let $\left(A, \succ_{A}, \prec_{A}\right)$ and $\left(B, \succ_{B}, \prec_{B}\right)$ be two dendriform algebras. Suppose that there are linear maps $l_{\succ_{A}}, r_{\succ_{A}}, l_{<_{A}}, r_{\succ_{A}}: A \rightarrow \mathfrak{g l}(B)$ and $l_{\succ_{B}}$, $r_{\succ_{B}}, l_{<_{B}}, r_{\succ_{B}}: B \rightarrow \operatorname{gr}(A)$ such that $\left(l_{\succ_{A}}, r_{\succ_{A}}, l_{\succ_{A}}, r_{\succ_{A}}\right)$ is a bimodule of $A$ and $\left(l_{\succ_{B}}, r_{\succ_{B}}, l_{\succ_{B}}, r_{\succ_{B}}\right)$ is a bimodule of $B$. If eqs. (35)-(52) are satisfied, then $\left(A, B, l_{\succ_{A}}, r_{\succ_{A}}, l_{\succ_{A}}, r_{\succ_{A}}, l_{\succ_{B}}, r_{\succ_{B}}, l_{\succ_{B}}, r_{\succ_{B}}\right)$ is called a matched pair of dendriform algebras.

Remark 3.2.6. Obviously $B$ is an ideal of $A \bowtie B$ if and only if $l_{\succ_{B}}=r_{\succ_{B}}=l_{\prec_{B}}=$

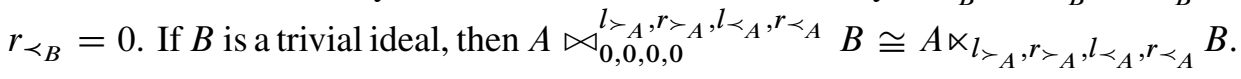

Corollary 3.2.7. Let $\left(A, B, l_{\succ_{A}}, r_{\succ_{A}}, l_{\succ_{A}}, r_{\succ_{A}}, l_{\succ_{B}}, r_{\succ_{B}}, l_{\prec_{B}}, r_{\prec_{B}}\right)$ be a matched pair of dendriform algebras. Then $\left(A, B, l_{\succ_{A}}+l_{\prec_{A}}, r_{\succ_{A}}+r_{\prec_{A}}, l_{\succ_{B}}+l_{<_{B}}, r_{\succ_{B}}+r_{\prec_{B}}\right)$ is a matched pair of the associated associative algebras $\left(A, *_{A}\right)$ and $\left(B, *_{B}\right)$.

Proof. In fact, the associated associative algebra $(A \bowtie B, *)$ is exactly the associative algebra obtained from the matched pair $\left(A, B, l_{A}, r_{A}, l_{B}, r_{B}\right)$ of associative algebras:

$$
(x+a) *(y+b)=x *_{A} y+l_{B}(a) y+r_{B}(b) x+a *_{B} b+l_{A}(x) b+r_{A}(y) a
$$

for all $x, y \in A, a, b \in B$, where $l_{A}=l_{\succ_{A}}+l_{\prec_{A}}, r_{A}=r_{\succ_{A}}+r_{\prec_{A}}, l_{B}=l_{\succ_{B}}+l_{\prec_{B}}$, $r_{B}=r_{\succ_{B}}+r_{\prec_{B}}$.

\section{Double constructions of Connes cocycles and an analogue of the classical Yang-Baxter equation}

\subsection{Connes cocycles and dendriform algebras}

Theorem 4.1.1. Let $(A, *)$ be an associative algebra and let $\omega$ be a non-degenerate Connes cocycle. Then there exists a compatible dendriform algebra structure $\succ, \prec$ on A given by

$$
\omega(x \succ y, z)=\omega(y, z * x), \quad \omega(x \prec y, z)=\omega(x, y * z) \quad \text { for all } x, y, z \in A .
$$

Proof. Define a linear map $T: A \rightarrow A^{*}$ by $\langle T(x), y\rangle=\omega(x, y)$ for all $x, y \in A$. Then $T$ is invertible and $T^{-1}$ is an $\mathcal{O}$-operator of the associative algebra $(A, *)$ associated to the bimodule $\left(R_{*}^{*}, L_{*}^{*}\right)$. By Corollary 3.1.3, there is a compatible dendriform algebra structure $\succ, \prec$ on $(A, *)$ given by

$$
x \succ y=T^{-1} R_{*}^{*}(x) T(y), \quad x \prec y=T^{-1} L_{*}^{*}(y) T(x)
$$

for all $x, y \in A$, which gives exactly eq. (53). 
Next, we turn to the double construction of the Connes cocycles. Let $\left(A, *_{A}\right)$ be an associative algebra and suppose that there is a associative algebra structure $*_{A} *$ on its dual space $A^{*}$. We construct an associative algebra structure on the direct sum $A \oplus A^{*}$ of the underlying vector spaces of $A$ and $A^{*}$ such that both $A$ and $A^{*}$ are subalgebras and the antisymmetric bilinear form on $A \oplus A^{*}$ given by eq. (7) is a Connes cocycle on $A \oplus A^{*}$. Such a construction is called a double construction of the Connes cocycle associated to $\left(A, *_{A}\right)$ and $\left(A^{*}, *^{*}\right)$ and we denote it by $\left(T(A)=A \bowtie A^{*}, \omega\right)$.

Corollary 4.1.2. Let $\left(T(A)=A \bowtie A^{*}, \omega\right)$ be a double construction of the Connes cocycle. Then there exists a compatible dendriform algebra structure $\succ, \prec$ on $T(A)$ defined by eq. (53). Moreover, $A$ and $A^{*}$ are dendriform subalgebras with this product.

Proof. The first half follows from Theorem 4.1.1. Let $x, y \in A$. Set $x>y=$ $a+b^{*}$, where $a \in A, b^{*} \in A^{*}$. Since $A$ is an associative subalgebra of $T(A)$ and $\omega(A, A)=\omega\left(A^{*}, A^{*}\right)=0$, we have

$$
\omega\left(b^{*}, A^{*}\right)=\omega\left(b^{*}, A\right)=\omega(x \succ y, A)=\omega(y, A * x)=0 .
$$

Therefore $b^{*}=0$ due to the nondependence of $\omega$. Hence $x \succ y=a \in A$. Similarly, $x \prec y \in A$. Thus $A$ is a dendriform subalgebra of $T(A)$ with the product $\succ, \prec$. By symmetry of $A$ and $A^{*}, A^{*}$ is also a dendriform subalgebra.

Definition 4.1.3. Let $\left(T\left(A_{1}\right)=A_{1} \bowtie A_{1}^{*}, \omega_{1}\right)$ and $\left(T\left(A_{2}\right)=A_{2} \bowtie A_{2}^{*}, \omega_{2}\right)$ be two double constructions of Connes cocycles. They are isomorphic if there exists an isomorphism of associative algebras $\varphi: T\left(A_{1}\right) \rightarrow T\left(A_{2}\right)$ satisfying the conditions

$$
\varphi\left(A_{1}\right)=A_{2}, \quad \varphi\left(A_{1}^{*}\right)=A_{2}^{*}, \quad \omega_{1}(x, y)=\varphi^{*} \omega_{2}(x, y)=\omega_{2}(\varphi(x), \varphi(y))
$$

for all $x, y \in A_{1}$.

Proposition 4.1.4. Two double constructions of Connes cocycles $\left(T\left(A_{1}\right)=A_{1} \bowtie\right.$ $\left.A_{1}^{*}, \omega_{1}\right)$ and $\left(T\left(A_{2}\right)=A_{2} \bowtie A_{2}^{*}, \omega_{2}\right)$ are isomorphic if and only if there exists $a$ dendriform algebra isomorphism $\varphi: T\left(A_{1}\right) \rightarrow T\left(A_{2}\right)$ satisfying eq. (54), where the dendriform algebra structures on $T\left(A_{1}\right)$ and $T\left(A_{2}\right)$ are given byeq. $(53)$, respectively.

Proof. This is straightforward.

Theorem 4.1.5. Let $\left(A, \succ_{A}, \prec_{A}\right)$ be a dendriform algebra and $\left(A, *_{A}\right)$ the associated associative algebra. Suppose that there is a dendriform algebra structure " $\succ_{A^{*}}$, $\prec_{A^{*}}$ " on its dual space $A^{*}$ and $\left(A^{*}, *^{*}\right)$ is the associated associative algebra. Then there exists a double construction of the Connes cocycle associated to $\left(A, *_{A}\right)$ and $\left(A, *_{A^{*}}\right)$ if and only if $\left(A, A^{*}, R_{\prec_{A}}^{*}, L_{\succ_{A}}^{*}, R_{\prec_{A^{*}}}^{*}, L_{\succ_{A^{*}}}^{*}\right)$ is a matched pair of the associative algebras. Moreover, every double construction of the Connes cocycle can be obtained in this way. 
Proof. The conclusion can be obtained by a similar proof as of Theorem 2.2.1.

Corollary 4.1.6. Let $(A, \succ, \prec)$ be a dendriform algebra and $\left(R_{\prec}^{*}, L_{\succ}^{*}\right)$ the bimodule of the associated associative algebra $(A, *)$. Then $\left(T(A)=A \ltimes_{R_{<}^{*}, L_{\succ}^{*}} A^{*}, \omega\right)$ is a double construction of the Connes cocycle. Conversely, let $\left(T(A)=A \bowtie A^{*}, \omega\right)$ be a double construction of the Connes cocycle. If $A^{*}$ is an ideal of $T(A)$, then $A^{*}$ is a trivial associative algebra and hence $T(A)$ is isomorphic to the semidirect $A \ltimes_{L_{T(A)}, R_{T(A)}} A^{*}$. Furthermore, this double construction of the Connes cocycle is isomorphic to the double construction of the Connes cocycle $\left(T(A)=A \ltimes_{R_{<}^{*}, L_{\succ}^{*}}\right.$ $\left.A^{*}, \omega\right)$ and the dendriform algebra structure on $A$ is given by $\omega$ from eq. (53).

Proof. By Remark 2.1.6, $\left(A, A^{*}, R_{<}^{*}, L_{\succ}^{*}, 0,0\right)$ with the associative algebra structure on $A^{*}$ being trivial is always a matched pair of associative algebras, the first half follows immediately. Conversely, if $A^{*}$ is an ideal, then, for any $a^{*}, b^{*} \in A^{*}$, it follows that if $T(A) * a^{*}, b^{*} * T(A) \in A^{*}$ then $\omega\left(a^{*} * b^{*}, T(A)\right)=-\omega(T(A) *$ $\left.a^{*}, b^{*}\right)-\omega\left(b^{*} * T(A), a^{*}\right)=0$. Thus $a^{*} * b^{*}=0$. Hence $T(A)$ is isomorphic to $A \ltimes_{L_{T(A)}, R_{T(A)}} A^{*}$. By Remark 2.1.6, it follows that that $\left(T(A)=A \bowtie A^{*}, \omega\right)$ is isomorphic to the double construction of the Connes cocycle $\left(T(A)=A \ltimes_{R_{\prec}^{*}, L_{\succ}^{*}}\right.$ $\left.A^{*}, \omega\right)$.

Theorem 4.1.7. Let $\left(A, \succ_{A}, \prec_{A}\right)$ be a dendriform algebra and $\left(A, *_{A}\right)$ the associated associative algebra. Suppose that there is a dendriform algebra structure " $\succ_{A^{*}}$, $\prec_{A^{*}}$ " on its dual space $A^{*}$ and $\left(A^{*}, *_{A^{*}}\right)$ is the associated associative algebra. Then $\left(A, A^{*}, R_{\succ_{A}}^{*}, L_{\succ_{A}}^{*}, R_{\succ_{A^{*}}}^{*}, L_{\succ_{A^{*}}}^{*}\right)$ is a matched pair of associative algebras if and only if

$$
\begin{aligned}
\left(A, A^{*}, R_{\succ_{A}}^{*}+R_{\succ_{A}}^{*},-L_{\succ_{A}}^{*},-R_{\succ_{A}}^{*}, L_{\succ_{A}}^{*}+L_{\succ_{A}}^{*},\right. & \\
& \left.R_{\succ_{A^{*}}^{*}}^{*}+R_{\succ_{A^{*}}}^{*},-L_{\succ_{A^{*}}}^{*},-R_{\succ_{A^{*}}}^{*}, L_{\succ_{A^{*}}}^{*}+L_{\succ_{A^{*}}}^{*}\right)
\end{aligned}
$$

is a matched pair of dendriform algebras.

Proof. The "if" part follows from Corollary 3.2.7. We need to prove the "only if" part. If $\left(A, A^{*}, R_{\succ_{A}}^{*}, L_{\succ_{A}}^{*}, R_{\succ_{A^{*}}}^{*}, L_{\succ_{A^{*}}}^{*}\right)$ is a matched pair of associative algebras, then $\left(A \bowtie_{R_{\succ_{A}}^{*}, L_{A^{*}}^{*}, L_{\succ_{A}}^{*}}^{R_{\succ^{*}}} A^{*}, \omega\right)$ is a double construction of the Connes cocycle. Hence there exists a compatible dendriform algebra structure on $A \bowtie_{R_{\succ^{*}}^{*} A^{*}, L_{\succ_{A}}^{*} A^{*}}^{R^{*}} A^{*}$ given by eq. (53). By a simple and direct computation, we show that $A$ and $A^{*}$ are its subalgebras and the other products are given by

$$
\begin{aligned}
& x \succ a^{*}=\left(R_{\succ_{A}}^{*}+R_{\succ_{A}}^{*}\right)(x) a^{*}-L_{\succ_{A^{*}}}^{*}\left(a^{*}\right) x, \\
& x \prec a^{*}=-R_{\succ_{A}}^{*}(x) a^{*}+\left(L_{\succ_{A^{*}}}^{*}+L_{\succ_{A^{*}}}^{*}\right)\left(a^{*}\right) x, \\
& a^{*} \succ x=\left(R_{\succ_{A^{*}}}^{*}+R_{\succ_{A^{*}}}^{*}\right)\left(a^{*}\right) x-L_{\succ_{A}}^{*}(x) a^{*}, \\
& a^{*} \prec x=-R_{\succ_{A^{*}}}^{*}\left(a^{*}\right) x+\left(L_{\succ_{A}}^{*}+L_{\succ_{A}}^{*}\right)(x) a^{*},
\end{aligned}
$$


for any $x \in A, a^{*} \in A^{*}$. Therefore

$$
\begin{aligned}
\left(A, A^{*}, R_{\succ_{A}}^{*}+R_{\succ_{A}}^{*},-L_{\succ_{A}}^{*},-R_{\succ_{A}}^{*}, L_{\succ_{A}}^{*}+L_{\succ_{A}}^{*},\right. & \\
& \left.R_{\succ_{A^{*}}}^{*}+R_{\succ_{A^{*}}}^{*},-L_{\succ_{A^{*}}}^{*},-R_{\succ_{A^{*}}}^{*}, L_{\succ_{A^{*}}}^{*}+L_{\succ_{A^{*}}}^{*}\right)
\end{aligned}
$$

is a matched pair of dendriform algebras.

\subsection{Dendriform D-bialgebras}

Theorem 4.2.1. Let $\left(A, \succ_{A}, \prec_{A}\right)$ be a dendriform algebra whose products are given by two linear maps $\beta_{\succ}^{*}, \beta_{<}^{*}: A \otimes A \rightarrow A$. Further suppose that there is a dendriform algebra structure " $\succ_{A^{*}}, \prec_{A^{*}}$ " on its dual space $A^{*}$ given by two linear maps $\Delta_{\succ}^{*}, \Delta_{\prec}^{*}: A^{*} \otimes A^{*} \rightarrow A^{*}$. Then $\left(A, A^{*}, R_{\succ_{A}}^{*}, L_{\succ_{A}}^{*}, R_{\succ_{A^{*}}}^{*}, L_{\succ_{A^{*}}}^{*}\right)$ is a matched pair of associative algebras if and only if the following equations hold for any $x, y \in A$ and $a^{*}, b^{*} \in A^{*}$ :

$$
\begin{aligned}
\Delta_{\prec}\left(x *_{A} y\right) & =\left(\mathrm{id} \otimes L_{\prec_{A}}(x)\right) \Delta_{\prec}(y)+\left(R_{A}(y) \otimes \mathrm{id}\right) \Delta_{\prec}(x), \\
\Delta_{\succ}\left(x *_{A} y\right) & =\left(\mathrm{id} \otimes L_{A}(x)\right) \Delta_{\succ}(y)+\left(R_{\prec_{A}}(y) \otimes \mathrm{id}\right) \Delta_{\succ}(x), \\
\beta_{\prec}\left(a^{*} *_{A^{*}} b^{*}\right)= & \left(\mathrm{id} \otimes L_{\prec_{A^{*}}}\left(a^{*}\right)\right) \beta_{\prec}\left(b^{*}\right)+\left(R_{A^{*}}\left(b^{*}\right) \otimes \mathrm{id}\right) \beta_{\prec}\left(a^{*}\right), \\
\beta_{\succ}\left(a^{*} *_{A^{*}} b^{*}\right)= & \left(\mathrm{id} \otimes L_{A^{*}}\left(a^{*}\right)\right) \beta_{\succ}\left(b^{*}\right)+\left(R_{\succ_{A^{*}}}\left(b^{*}\right) \otimes \mathrm{id}\right) \beta_{\succ}\left(a^{*}\right), \\
\left(L_{A}(x) \otimes \mathrm{id}\right. & \left.-\mathrm{id} \otimes R_{\prec_{A}}(x)\right) \Delta_{\prec}(y) \\
& +\sigma\left[\left(L_{\succ_{A}}(y) \otimes-\mathrm{id} \otimes R_{A}(y)\right) \Delta_{\succ}(x)\right]=0, \\
\left(L_{A^{*}}\left(a^{*}\right) \otimes \mathrm{id}-\mathrm{id} \otimes R_{\prec_{A}}\left(a^{*}\right)\right) \beta_{\prec}\left(b^{*}\right) & \\
& +\sigma\left[\left(L_{\succ_{A^{*}}}\left(b^{*}\right) \otimes-\mathrm{id} \otimes R_{A^{*}}\left(b^{*}\right)\right) \beta_{\succ}\left(a^{*}\right)\right]=0,
\end{aligned}
$$

where $L_{A}=L_{\succ_{A}}+L_{\succ_{A}}, R_{A}=R_{\succ_{A}}+R_{\succ_{A}}, L_{A^{*}}=L_{\succ_{A^{*}}}+L_{\prec_{A^{*}}}, R_{A^{*}}=$ $R_{\succ_{A^{*}}}+R_{\prec_{A^{*}}}$.

Proof. Let $\left\{e_{1}, \ldots, e_{n}\right\}$ be a basis of $A$ and $\left\{e_{1}^{*}, \ldots, e_{n}^{*}\right\}$ its dual basis. Set

$$
\begin{aligned}
e_{i} \succ_{A} e_{j} & =\sum_{k=1}^{n} a_{i j}^{k} e_{k}, & e_{i} \prec_{A} e_{j} & =\sum_{k=1}^{n} b_{i j}^{k} e_{k}, \\
e_{i}^{*} \succ_{A^{*}} e_{j}^{*} & =\sum_{k=1}^{n} c_{i j}^{k} e_{k}^{*}, & e_{i}^{*} \prec_{A^{*}} e_{j}^{*} & =\sum_{k=1}^{n} d_{i j}^{k} e_{k}^{*} .
\end{aligned}
$$

Therefore the coefficient of $e_{l}^{*}$ in

$$
R_{\succ_{A}}^{*}\left(e_{i}\right)\left(e_{j}^{*} *_{A^{*}} e_{k}^{*}\right)=R_{\succ_{A}}^{*}\left(L_{\succ_{A^{*}}}^{*}\left(e_{j}^{*}\right) e_{i}\right) e_{k}^{*}+R_{\succ_{A}}^{*}\left(e_{i}\right) e_{j}^{*} *_{A^{*}} e_{k}^{*}
$$

gives the following relation (for any $i, j, k, l$ )

$$
\sum_{m=1}^{n} b_{l i}^{m}\left(c_{j k}^{m}+d_{j k}^{m}\right)=\sum_{m=1}^{n}\left[c_{j m}^{i} b_{l m}^{k}+b_{m i}^{j}\left(c_{m k}^{l}+d_{m k}^{l}\right)\right],
$$


which is precisely the relation given by the coefficient of $e_{l}^{*} \otimes e_{i}^{*}$ in

$$
\beta_{\prec}\left(e_{j}^{*} *_{A^{*}} e_{k}^{*}\right)=\left(R_{A^{*}}\left(e_{k}^{*}\right) \otimes \mathrm{id}\right) \beta_{\prec}\left(e_{j}^{*}\right)+\left(\mathrm{id} \otimes L_{\succ_{A^{*}}}\left(e_{j}^{*}\right)\right) \beta_{\prec}\left(e_{k}^{*}\right) .
$$

So eq. (9) in the case $l_{A}=R_{\prec_{A}}^{*}, r_{A}=L_{\succ_{A}}^{*}, l_{B}=l_{A^{*}}=R_{\prec_{A^{*}}}^{*}, r_{B}=r_{A^{*}}=L_{\succ_{A^{*}}}^{*}$ is eq. (57). Similarly, in this situation, we have the following correspondences:

$$
\begin{aligned}
& \text { eq. (10) } \Longleftrightarrow \text { eq. (58), } \quad \text { eq. (11) } \Longleftrightarrow \text { eq. (55), eq. (12) } \Longleftrightarrow \text { eq. (56), } \\
& \text { eq. (13) } \Longleftrightarrow \text { eq. (60), eq. (14) } \Longleftrightarrow \text { eq. (59). }
\end{aligned}
$$

Therefore the conclusion holds due to Theorem 2.1.4.

Definition 4.2.2. Let $A$ be a vector space. A dendriform D-bialgebra structure on $A$ is a set of linear maps $\left(\Delta_{\prec}, \Delta_{\succ}, \beta_{\prec}, \beta_{\succ}\right)$ such that $\Delta_{\prec}, \Delta_{\succ}: A \rightarrow A \otimes A$,

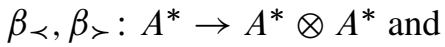

(a) $\left(\Delta_{\prec}^{*}, \Delta_{\succ}^{*}\right): A^{*} \otimes A^{*} \rightarrow A^{*}$ defines a dendriform algebra structure $\left(\succ_{A^{*}}, \prec_{A^{*}}\right)$ on $A^{*}$;

(b) $\left(\beta_{\prec}^{*}, \beta_{\succ}^{*}\right): A \otimes A \rightarrow A$ defines a dendriform algebra structure $\left(\succ_{A}, \prec_{A}\right)$ on $A$;

(c) eqs. (55)-(60) are satisfied.

We also denote it by $\left(A, A^{*}, \Delta_{\succ}, \Delta_{\prec}, \beta_{\succ}, \beta_{\prec}\right)$ or simply $\left(A, A^{*}\right)$.

Remark 4.2.3. In fact, the notions of dendriform bialgebra ([LR1]-[LR2], [Ron], [A4]) and bidendriform bialgebra ([F2]), which are the special dendriform bialgebras, were already introduced. We use the terminology "D-bialgebra" in order to express its relation with the double construction. All of these bialgebras are dendriform algebras equipped with coassociative cooperations satisfying some (different) compatibility relations. We would like to point out that the dendriform D-bialgebras are quite different from the other types of bialgebras. For example, one of the differences is that the term $a \otimes b$ appears in both $\Delta_{\prec}(a * b)$ and $\Delta_{\succ}(a * b)$ in a bidendriform bialgebra, whereas it does not appear in a dendriform D-bialgebra.

Theorem 4.2.4. Let $\left(A, \prec_{A}, \succ_{A}\right)$ and $\left(A^{*}, \prec_{A^{*},} \succ_{A^{*}}\right)$ be two dendriform algebras. Let $\left(A, *_{A}\right)$ and $\left(A^{*}, *^{*}\right)$ be the associated associative algebras respectively. Then the following conditions are equivalent.

(1) There is a double construction of the Connes cocycle associated to $\left(A, *_{A}\right)$ and $\left(A, * A^{*}\right)$.

(2) ( $\left.A, A^{*}, R_{\succ_{A}}^{*}, L_{\succ_{A}}^{*}, R_{\succ_{A^{*}}}^{*}, L_{\succ_{A^{*}}}^{*}\right)$ is a matched pair of the associative algebras.

(3) $\left(A, A^{*}, R_{\succ_{A}}^{*}+R_{\succ_{A}}^{*},-L_{\prec_{A}}^{*},-R_{\succ_{A}}^{*}, L_{\succ_{A}}^{*}+L_{\prec_{A}}^{*}, R_{\succ_{A^{*}}}^{*}+R_{\prec_{A^{*}}}^{*},-L_{\prec_{A^{*}}}^{*}\right.$, $-R_{\succ_{A^{*}}}^{*}, L_{\succ_{A^{*}}}^{*}+L_{\prec_{A^{*}}}^{*}$ ) is a matched pair of dendriform algebras.

(4) $\left(A, A^{*}\right)$ is a dendriform D-bialgebra.

Proof. This follows from Theorems 4.1.5, 4.1.7 and 4.2.1. 
Definition 4.2.5. Let $\left(A, A^{*}, \Delta_{\succ}, \Delta_{\prec}, \beta_{\succ}, \beta_{\prec}\right)$ and $\left(B, B^{*}, \Delta_{\succ}, \Delta_{\prec}, \beta_{\succ}, \beta_{\prec}\right)$ be two dendriform D-bialgebras. A homomorphism of dendriform D-bialgebras $\varphi: A \rightarrow$ $B$ is a homomorphism of dendriform algebras such that $\varphi^{*}: B^{*} \rightarrow A^{*}$ is also a homomorphism of dendriform algebras, that is, $\varphi$ satisfies

$$
\begin{array}{cl}
(\varphi \otimes \varphi) \Delta_{\succ}(x)=\Delta_{\succ}(\varphi(x)), & (\varphi \otimes \varphi) \Delta_{\prec}(x)=\Delta_{\prec}(\varphi(x)), \\
\left(\varphi^{*} \otimes \varphi^{*}\right) \beta_{\succ}\left(a^{*}\right)=\beta_{\succ}\left(\varphi^{*}\left(a^{*}\right)\right), & \left(\varphi^{*} \otimes \varphi^{*}\right) \beta_{\prec}\left(a^{*}\right)=\beta_{\prec}\left(\varphi^{*}\left(a^{*}\right)\right),
\end{array}
$$

for any $x \in A, a^{*} \in B^{*}$. An isomorphism of dendriform D-bialgebras is an invertible homomorphism of dendriform D-bialgebras.

Proposition 4.2.6. Two double constructions of Connes cocycles are isomorphic if and only if their corresponding dendriform D-bialgebras are isomorphic.

Proof. It follows from a similar proof as of Proposition 2.2.10.

Example 4.2.7. Let $\left(A, A^{*}, \Delta_{\succ}, \Delta_{\prec}, \beta_{\succ}, \beta_{\prec}\right)$ be a dendriform D-bialgebra. Then its dual $\left(A^{*}, A, \beta_{\succ}, \beta_{\prec}, \Delta_{\succ}, \Delta_{\prec}\right)$ is also a dendriform D-bialgebra.

Example 4.2.8. Let $\left(A, \succ_{A}, \prec_{A}\right)$ be a dendriform algebra. If the dendriform algebra structure on $A^{*}$ is trivial, then $\left(A, A^{*}, 0,0, \beta_{\succ}, \beta_{\prec}\right)$ is a dendriform D-bialgebra. And

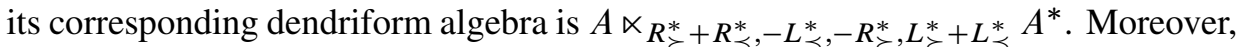
its corresponding double construction of the Connes cocycle is just the semidirect sum $A \ltimes_{R_{\succ}^{*}, L_{\succ}^{*}} A^{*}$ with the bilinear form $\omega$ given by eq. (7). Dually, if $A$ is a trivial dendriform algebra, then the dendriform D-bialgebra structures on $A$ are in one-to-one correspondence with the dendriform algebra structures on $A^{*}$.

Example 4.2.9. Let $\left(A, A^{*}\right)$ be a dendriform D-bialgebra. In the next subsection, we will prove that there exists a canonical dendriform D-bialgebra structure on the direct sum $A \oplus A^{*}$ of the underlying vector spaces of $A$ and $A^{*}$.

4.3. Coboundary dendriform D-bialgebras. In Theorem 4.2.1 we showed that both $\Delta_{\succ}$ and $\Delta_{\prec}\left(\beta_{\succ}\right.$ and $\beta_{\prec}$, respectively $)$ are the 1-cocycles of the associated associative algebra $\left(A, *_{A}\right)$ (resp. $\left.\left(A^{*}, *_{A^{*}}\right)\right)$. So it is natural to consider the special case that they are 1-coboundaries or principal derivations, as we did in Section 2.3.

Let $(A, \succ, \prec)$ be a dendriform algebra and $r_{\succ}, r_{\prec} \in A \otimes A$. Set

$$
\begin{gathered}
\Delta_{\succ}(x)=\left(\mathrm{id} \otimes L(x)-R_{\prec}(x) \otimes \mathrm{id}\right) r_{\succ}, \\
\Delta_{\prec}(x)=\left(\mathrm{id} \otimes L_{\succ}(x)-R(x) \otimes \mathrm{id}\right) r_{\prec}
\end{gathered}
$$

for any $x \in A$. It is obvious that $\Delta_{\succ}$ satisfies eq. (55) and $\Delta_{\prec}$ satisfies eq. (56). Moreover, by eq. (59), it follows that

$$
\left(L(x) \otimes \mathrm{id}-\mathrm{id} \otimes R_{\prec}(x)\right)\left(\mathrm{id} \otimes L_{\succ}(y)-R(y) \otimes \mathrm{id}\right)\left(r_{\prec}+\sigma\left(r_{\succ}\right)\right)=0
$$

for all $x, y \in A$. Therefore $\left(A, \Delta_{\succ}, \Delta_{\prec}, \beta_{\succ}, \beta_{\prec}\right)$ is a dendriform D-bialgebra if and only if the following conditions are satisfied: 
(1) $\Delta_{\succ}^{*}, \Delta_{\prec}^{*}: A^{*} \otimes A^{*} \rightarrow A^{*}$ defines a dendriform algebra structure on $A^{*}$.

(2) $\beta_{\succ}, \beta_{\prec}$ satisfy eqs. (57), (58) and (60), where the dendriform algebra structure on $A^{*}$ is given by (1).

Proposition 4.3.1. Let $(A, \succ, \prec)$ be a dendriform algebra whose products are given by two linear maps $\beta_{\succ}^{*}, \beta_{\prec}^{*}: A \otimes A \rightarrow A$ and $r_{\succ}, r_{\prec} \in A \otimes A$. Suppose there exists a dendriform algebra structure " $\succ_{A^{*},} \prec_{A^{*}}$ " on $A^{*}$ given by $\Delta_{\succ}^{*}, \Delta_{\prec}^{*}: A^{*} \otimes A^{*} \rightarrow A^{*}$, where $\Delta_{\succ}$ and $\Delta_{\prec}$ are two linear maps given by eqs. (61) and (62), respectively. Then

(1) Eq. (57) holds if and only if $r_{\succ}, r_{\prec}$ satisfy

$$
\left[R_{\prec}(x) \otimes L_{\succ}(y)-\mathrm{id} \otimes L_{\succ}(y \prec x)-R_{\prec}(y \succ x) \otimes \mathrm{id}\right]\left(r_{\succ}+r_{\prec}\right)=0
$$

for all $x, y \in A$.

(2) Eq. (58) holds if and only if $r_{\succ}, r_{\prec}$ satisfy eq. (64).

(3) Eq. (60) holds if and only if $r_{\succ}, r_{\prec}$ satisfy

$$
\begin{aligned}
& {\left[L_{\succ}(x) \otimes \mathrm{id}-\mathrm{id} \otimes R_{\prec}(x)\right]\left[-\mathrm{id} \otimes L_{\succ}(y)+R_{\prec}(y) \otimes \mathrm{id}\right]\left(r_{\prec}+r_{\succ}\right)} \\
& \quad+\left[L_{\succ}(x) \otimes \mathrm{id}-\mathrm{id} \otimes R_{\prec}(x)\right]\left[R_{\succ}(y) \otimes \mathrm{id}\left(r_{\prec}+\sigma\left(r_{\succ}\right)\right)\right. \\
& \left.\quad-\mathrm{id} \otimes L_{\prec}(y)\left(\sigma\left(r_{\prec}\right)+r_{\succ}\right)\right]=0
\end{aligned}
$$

for any $x, y \in A$.

Proof. Let $\left\{e_{1}, \ldots, e_{n}\right\}$ be a basis of $A$ and $\left\{e_{1}^{*}, \ldots, e_{n}^{*}\right\}$ its dual basis. Set

$$
\begin{aligned}
r_{\prec} & =\sum_{i, j} a_{i j} e_{i} \otimes e_{j}, & r_{\succ} & =\sum_{i, j} b_{i j} e_{i} \otimes e_{j}, \\
e_{i} \succ e_{j} & =\sum_{k=1}^{n} a_{i j}^{k} e_{k}, & e_{i} \prec e_{j} & =\sum_{k=1}^{n} b_{i j}^{k} e_{k}, \\
e_{i}^{*} \succ e_{j}^{*} & =\sum_{k=1}^{n} c_{i j}^{k} e_{k}^{*}, & e_{i}^{*} \prec e_{j}^{*} & =\sum_{k=1}^{n} d_{i j}^{k} e_{k}^{*} .
\end{aligned}
$$

By eqs. (61) and (62), we have (for any $i, k, l$ )

$$
\begin{aligned}
& c_{k l}^{i}=\sum_{m=1}^{n}\left[b_{k m}\left(a_{i m}^{l}+b_{i m}^{l}\right)-b_{m l} b_{m i}^{k}\right], \\
& d_{k l}^{i}=\sum_{m=1}^{n}\left[a_{k m} a_{i m}^{l}-a_{m l}\left(a_{m i}^{k}+b_{m i}^{k}\right)\right] .
\end{aligned}
$$

(1) Eq. (57) holds (taking $a^{*}=e_{i}^{*}, b^{*}=e_{j}^{*}$ ) if and only if (for any $i, j, m, t$ )

$$
\sum_{k=1}^{n}\left(c_{i j}^{k}+d_{i j}^{k}\right) b_{m t}^{k}=\sum_{k=1}^{n}\left[b_{m k}^{j} c_{i k}^{t}+b_{k t}^{i}\left(c_{k j}^{m}+d_{k j}^{m}\right)\right] .
$$


Substituting eq. (66) into the above equation and after rearranging the terms suitably, we have

$$
(\mathrm{F} 1)+(\mathrm{F} 2)+(\mathrm{F} 3)+(\mathrm{F} 4)+(\mathrm{F} 5)+(\mathrm{F} 6)=0,
$$

where

$$
\begin{array}{rlrl}
(\mathrm{F} 1) & =\sum_{k, l}\left(a_{k l}+b_{k l}\right)\left(a_{m l}^{j} b_{k t}^{i}\right), & (\mathrm{F} 2) & =\sum_{k, l}\left(b_{k l} b_{k t}^{i} b_{m l}^{j}-b_{l k} b_{l n}^{i} b_{m k}^{j}\right) ; \\
(\mathrm{F} 3) & =\sum_{k, l}\left(a_{i l}+b_{i l}\right)\left(-a_{k l}^{j} b_{m t}^{k}\right), & (\mathrm{F} 4) & =\sum_{k, l} b_{i l}\left[b_{m k}^{j}\left(a_{t l}^{k}+b_{t l}^{k}\right)-b_{m t}^{k} b_{k l}^{j}\right] ; \\
(\mathrm{F} 5) & =\sum_{k, l}\left(a_{l j}+b_{l j}\right)\left(b_{m t}^{k} b_{l k}^{i}-b_{k t}^{i} b_{l m}^{k}\right),(\mathrm{F} 6)=\sum_{k, l} a_{l j}\left(a_{l k}^{i} b_{m t}^{k}-a_{l m}^{k} b_{k t}^{i}\right) .
\end{array}
$$

Here $(\mathrm{F} 1)$ is the coefficient of $e_{i} \otimes e_{j}$ in $\left[R_{\prec}\left(e_{t}\right) \otimes L_{\succ}\left(e_{m}\right)\right]\left(r_{\succ}+r_{\prec}\right)$;

$(\mathrm{F} 2)=0$ by interchanging the indices $k$ and $l$;

(F3) is the coefficient of $e_{i} \otimes e_{j}$ in $-\left[\mathrm{id} \otimes L_{\succ}\left(e_{m} \prec e_{t}\right)\right]\left(r_{\succ}+r_{\prec}\right)$;

$(\mathrm{F} 4)=0$ since the term in the bracket is the coefficient of $e_{j}$ in

$$
e_{m} \prec\left(e_{t} \succ e_{l}+e_{t} \prec e_{l}\right)-\left(e_{m} \prec e_{n}\right) \prec e_{l}=0 ;
$$

(F5) is the coefficient of $e_{i} \otimes e_{j}$ in $-\left[R_{\prec}\left(e_{m} \succ e_{t}\right) \otimes \mathrm{id}\right]\left(r_{\succ}+r_{\prec}\right)$.

$(\mathrm{F} 6)=0$ since the term in the bracket is the coefficient of $e_{i}$ in

$$
e_{l} \succ\left(e_{m} \prec e_{t}\right)-\left(e_{l} \succ e_{m}\right) \prec e_{t}=0 \text {. }
$$

Therefore we have

$$
\left[R_{\prec}\left(e_{t}\right) \otimes L_{\succ}\left(e_{m}\right)-\mathrm{id} \otimes L_{\succ}\left(e_{m} \prec e_{t}\right)-R_{\prec}\left(e_{m} \succ e_{t}\right) \otimes \mathrm{id}\right]\left(r_{\succ}+r_{\prec}\right)=0 .
$$

(2) Similarly, eq. (58) holds if and only if $r_{\succ}, r_{\prec}$ satisfy eq. (64). In fact, comparing with the proof in (1), the difference appears in $(\mathrm{F} 2)^{\prime},(\mathrm{F} 4)^{\prime}$ and $(\mathrm{F} 6)^{\prime}$, where

$(\mathrm{F} 2)^{\prime}=\sum_{k, l}\left(a_{m k}^{j} a_{l t}^{i}-a_{k t}^{i} a_{m l}^{j}\right)=0$ by interchanging the indices $k$ and $l$;

$(\mathrm{F} 4)^{\prime}=\sum_{k, l} b_{i l}\left(a_{m t}^{k} b_{k l}^{j}-a_{m k}^{j} b_{t l}^{k}\right)=0$ since the term in the bracket is the coefficient of $e_{j}$ in

$$
\left(e_{m} \succ e_{t}\right) \prec e_{l}-e_{m} \succ\left(e_{t} \prec e_{l}\right)=0 ;
$$

$(\mathrm{F} 6)^{\prime}=\sum_{k, l} a_{l j}\left[a_{k t}^{i}\left(a_{l m}^{k}+b_{l m}^{k}\right)-a_{m t}^{k} a_{l k}^{i}\right]=0$ since the term in the bracket is the coefficient of $e_{i}$ in $-e_{l} \succ\left(e_{m} \succ e_{t}\right)+\left(e_{l} \succ e_{m}+e_{l} \prec e_{m}\right) \succ e_{t}=0$.

(3) Eq. (60) holds (taking $a^{*}=e_{i}^{*}, b^{*}=e_{j}^{*}$ ) if and only if (for any $i, j, m, t$ )

$$
\sum_{l=1}^{n}\left[\left(c_{i l}^{m}+d_{i l}^{m}\right) b_{l t}^{j}-b_{m l}^{j} d_{l i}^{t}+a_{l m}^{i} c_{j l}^{t}-a_{t l}^{i}\left(c_{l j}^{m}+d_{l j}^{m}\right)\right]=0 .
$$

Substituting eq. (66) into the above equation and after rearranging the terms suitably, we have

$$
(\mathrm{F} 1)+(\mathrm{F} 2)+(\mathrm{F} 3)+(\mathrm{F} 4)+(\mathrm{F} 5)+(\mathrm{F} 6)+(\mathrm{F} 7)+(\mathrm{F} 8)+(\mathrm{F} 9)+(\mathrm{F} 10)=0,
$$


where

$$
\begin{aligned}
& (\mathrm{F} 1)=\sum_{k, l}\left(a_{k l}+b_{k l}\right)\left(-b_{l t}^{j} b_{k m}^{i}\right) \Longrightarrow-R_{\prec}\left(e_{m}\right) \otimes R_{\prec}\left(e_{t}\right)\left(r_{\succ}+r_{\prec}\right), \\
& (\mathrm{F} 2)=\sum_{k, l}\left(a_{l k}+b_{l k}\right)\left(-a_{m k}^{j} a_{t l}^{i}\right) \Longrightarrow-L_{\succ}\left(e_{t}\right) \otimes L_{\succ}\left(e_{m}\right)\left(r_{\succ}+r_{\prec}\right), \\
& (\mathrm{F} 3)=\sum_{k, l}\left(a_{k l}+b_{l k}\right)\left(-a_{k m}^{i} b_{l t}^{j}\right) \Longrightarrow-R_{\succ}\left(e_{m}\right) \otimes R_{\prec}\left(e_{t}\right)\left(\sigma\left(r_{\succ}\right)+r_{\prec}\right), \\
& (\mathrm{F} 4)=\sum_{k, l}\left(a_{l k}+b_{k l}\right)\left(-a_{m k}^{j} b_{t l}^{j}\right) \Longrightarrow-L_{\succ}\left(e_{t}\right) \otimes L_{\prec}\left(e_{m}\right)\left(r_{\succ}+\sigma\left(r_{\prec}\right)\right), \\
& (\mathrm{F} 5)=\sum_{k, l}\left(a_{i k}+b_{i k}\right) a_{m k}^{l} b_{l t}^{j} \Longrightarrow \mathrm{id} \otimes R_{\prec}\left(e_{t}\right) L_{\succ}\left(e_{m}\right)\left(r_{\succ}+r_{\prec}\right), \\
& (\mathrm{F} 6)=\sum_{k, l} a_{k i}\left(a_{k t}^{l}+b_{k t}^{l}\right) b_{m l}^{j} \Longrightarrow \mathrm{id} \otimes R_{\prec}\left(e_{t}\right) L_{\prec}\left(e_{m}\right)\left(\sigma\left(r_{\prec}\right)\right), \\
& (\mathrm{F} 7)=\sum_{k, l} b_{i k} b_{l t}^{j} b_{m k}^{l} \Longrightarrow \mathrm{id} \otimes R_{\prec}\left(e_{t}\right) L_{\prec}\left(e_{m}\right)\left(r_{\succ}\right), \\
& (\mathrm{F} 8)=\sum_{k, l}\left(a_{k j}+b_{k j}\right) a_{t l}^{i} b_{k m}^{l} \Longrightarrow L_{\succ}\left(e_{t}\right) R_{\prec}\left(e_{m}\right) \otimes \operatorname{id}\left(r_{\succ}+r_{\prec}\right), \\
& (\mathrm{F} 9)=\sum_{k, l} a_{k j} a_{k m}^{l} a_{t l}^{i} \Longrightarrow L_{\succ}\left(e_{t}\right) R_{\succ}\left(e_{m}\right) \otimes \operatorname{id}\left(r_{\prec}\right), \\
& (\mathrm{F} 10)=\sum_{k, l} b_{j k} a_{l m}^{i}\left(a_{t k}^{l}+b_{t k}^{l}\right) \Longrightarrow L_{\succ}\left(e_{t}\right) R_{\succ}\left(e_{m}\right) \otimes \mathrm{id}\left(\sigma\left(r_{\succ}\right)\right) .
\end{aligned}
$$

Therefore eq. (65) holds. [F2]).

By the definition of a dendriform algebra, we have the following conclusion (cf.

Lemma 4.3.2. Let $A$ be a vector space and let $\Delta_{\succ}, \Delta_{\prec}: A \otimes A \rightarrow A$ be two linear maps. Then $\Delta_{\succ}^{*}, \Delta_{\prec}^{*}: A^{*} \otimes A^{*} \rightarrow A^{*}$ define a dendriform algebra structure on $A^{*}$ if and only if the following conditions are satisfied:

$$
\begin{aligned}
& \left(\Delta_{\prec} \otimes \mathrm{id}\right) \Delta_{\prec}=\left(\operatorname{id} \otimes\left(\Delta_{\succ}+\Delta_{\prec}\right)\right) \Delta_{\prec}, \\
& \left(\mathrm{id} \otimes \Delta_{\prec}\right) \Delta_{\succ}=\left(\Delta_{\succ} \otimes \mathrm{id}\right) \Delta_{\prec}, \\
& \left(\mathrm{id} \otimes \Delta_{\succ}\right) \Delta_{\succ}=\left(\left(\Delta_{\succ}+\Delta_{\prec}\right) \otimes \mathrm{id}\right) \Delta_{\succ} .
\end{aligned}
$$

Proposition 4.3.3. Let $(A, \succ, \prec)$ be a dendriform algebra and $r_{\succ}, r_{\prec} \in A \otimes A$. Define $\Delta_{\succ}, \Delta_{\prec}: A \rightarrow A \otimes A$ by eqs. (61) and (62). Then $\Delta_{\succ}^{*}, \Delta_{\prec}^{*}: A^{*} \otimes A^{*} \rightarrow A^{*}$ define a dendriform algebra structure on $A^{*}$ if and only if the following equations 
are satisfied (for any $x \in A$ )

$$
\begin{aligned}
& (R(x) \otimes \mathrm{id} \otimes \mathrm{id})\left[\left(r_{\prec, 12} * r_{\prec, 13}+r_{\prec, 13} \prec r_{\succ, 23}-r_{\prec, 23} \succ r_{\prec, 12}\right)\right. \\
& \left.\quad+r_{\prec, 13} \succ\left(r_{\prec, 23}+r_{\succ, 23}\right)-\left(r_{\prec, 23}+r_{\succ, 23}\right) \prec r_{\prec, 12}\right] \\
& \quad+\left(r_{\prec, 23}+r_{\succ, 23}\right) \prec\left[\left(\mathrm{id} \otimes L_{\prec}(x) \otimes \mathrm{id}\right) r_{\prec, 12}\right] \\
& \quad+\left(\mathrm{id} \otimes \mathrm{id} \otimes L_{\succ}(x)\right)\left(-r_{\prec, 12} * r_{\prec, 13}-r_{\prec, 13} \prec r_{\succ, 23}+r_{\prec, 23} \succ r_{\prec, 12}\right) \\
& \quad-\left[\left(\mathrm{id} \otimes \mathrm{id} \otimes L_{\succ}(x)\right) r_{13}\right] \succ\left(r_{\succ, 23}+r_{\prec, 23}\right)=0 ;
\end{aligned}
$$

$$
\begin{aligned}
& \left(R_{\prec}(x) \otimes \mathrm{id} \otimes \mathrm{id}\right)\left(r_{\prec, 23} * r_{\succ, 12}-r_{\succ, 12} \prec r_{\prec, 13}-r_{\succ, 13} \succ r_{\prec, 23}\right) \\
& \quad-\left(\mathrm{id} \otimes \mathrm{id} \otimes L_{\succ}(x)\right)\left(r_{\prec, 23} * r_{\succ, 12}-r_{\succ, 12} \prec r_{\prec, 13}-r_{\succ, 13} \succ r_{\prec, 23}\right)=0 ;
\end{aligned}
$$

$\left(R_{\prec}(x) \otimes \mathrm{id} \otimes \mathrm{id}\right)\left(-r_{\succ, 13} * r_{\succ, 23}+r_{\succ, 23} \prec r_{\succ, 12}-r_{\prec, 12} \succ r_{\succ, 13}\right)$

$$
\begin{aligned}
& -\left(r_{\succ, 12}+r_{\prec, 12}\right) \prec\left[\left(R_{\prec}(x) \otimes \mathrm{id} \otimes 1\right) r_{\succ, 13}\right] \\
& +\left[\left(\mathrm{id} \otimes R_{\prec}(x) \otimes \mathrm{id}\right) r_{\succ, 23}\right] \succ\left(r_{\succ, 12}+r_{\prec, 12}\right)
\end{aligned}
$$

$$
\begin{aligned}
& +(\mathrm{id} \otimes \mathrm{id} \otimes L(x))\left[r_{\succ, 13} * r_{\succ, 23}-r_{\succ, 23} \prec r_{\succ, 12}+r_{\prec, 12} \succ r_{\succ, 13}\right. \\
& \left.+\left(r_{\succ, 12}+r_{\prec, 12}\right) \prec r_{\succ, 13}-r_{\succ, 23} \succ\left(r_{\succ, 12}+r_{\prec, 12}\right)\right]=0 .
\end{aligned}
$$

The operation between two $r$ s is given in an obvious and similar way as eq. (4).

Proof. We need to prove that eqs. (67)-(69) are equivalent to eqs. (70)-(72), respectively. Here we only give an explicit proof that eq. (70) holds if and only if eq. (67) holds since the proof of the other two equations is similar. Let $x \in A$. After rearranging the terms suitably, we divide eq. (67) into three parts:

$$
\left(\Delta_{\prec} \otimes \mathrm{id}\right) \Delta_{\prec}(x)-\left(\mathrm{id} \otimes\left(\Delta_{\succ}+\Delta_{\prec}\right)\right) \Delta_{\prec}(x)=(\mathrm{F} 1)+(\mathrm{F} 2)+(\mathrm{F} 3),
$$

where

$$
\begin{aligned}
(\mathrm{F} 1)= & \sum_{i, j}\left\{( a _ { i } \succ x + a _ { i } \prec x ) \otimes \left[a_{j} \otimes b_{i} \succ b_{j}-\left(a_{j} \succ b_{i}+a_{j} \prec b_{i}\right) \otimes b_{j}\right.\right. \\
& \left.+c_{j} \otimes\left(b_{i} \succ d_{j}+b_{i} \prec d_{j}\right)-c_{j} \prec b_{i} \otimes d_{j}\right]+\left[a_{j} \succ\left(a_{i} \succ x+a_{i} \prec x\right)\right. \\
& \left.\left.+a_{j} \prec\left(a_{i} \succ x+a_{i} \prec x\right)\right] \otimes b_{j} \otimes b_{i}\right\}, \\
(\mathrm{F} 2)= & \sum_{i, j}\left\{a_{i} \otimes\left[a_{j} \succ\left(x \succ b_{i}\right)+a_{j} \prec\left(x \succ b_{i}\right)\right] \otimes b_{j}\right. \\
& \left.+a_{i} \otimes c_{j} \prec\left(x \succ b_{i}\right) \otimes d_{j}-a_{j} \otimes\left(a_{i} \succ x+a_{i} \prec x\right) \succ b_{j} \otimes b_{i}\right\}, \\
(\mathrm{F} 3)= & \sum_{i, j}\left\{\left[a_{i} \otimes\left(a_{i} \succ b_{j}\right)-\left(a_{j} \succ a_{i}+a_{j} \prec a_{i}\right) \otimes b_{j}\right] \otimes\left(x \succ b_{i}\right)-a_{i} \otimes a_{j}\right. \\
& \left.\otimes\left[\left(x \succ b_{i}\right) \succ b_{j}\right]-a_{i} \otimes c_{j} \otimes\left[\left(x \succ b_{i}\right) \succ d_{j}+\left(x \succ b_{i}\right) \prec d_{j}\right]\right\} .
\end{aligned}
$$


On the other hand,

$(\mathrm{F} 1 \mathrm{a})=(R(x) \otimes \mathrm{id} \otimes \mathrm{id})\left(r_{\prec, 12} * r_{\prec, 13}\right)$

$$
\begin{aligned}
& =\sum_{i, j}\left[\left(a_{i} * a_{j}\right) * x \otimes b_{i} \otimes b_{j}\right] \\
& \left.=\sum_{i, j}\left[a_{j} \succ\left(a_{i} \succ x+a_{i} \prec x\right)+a_{j} \prec\left(a_{i} \succ x+a_{i} \prec x\right)\right] \otimes b_{j} \otimes b_{i}\right],
\end{aligned}
$$

$(\mathrm{F} 1 \mathrm{~b})=(R(x) \otimes \mathrm{id} \otimes \mathrm{id})\left(r_{\prec, 13} \prec r_{\succ, 23}\right)$

$$
\begin{aligned}
& =\sum_{i, j}\left[\left(a_{i} * x\right) \otimes c_{j} \otimes\left(b_{i} \prec d_{j}\right)\right] \\
& =\sum_{i, j}\left[\left(a_{i} \succ x+a_{i} \prec x\right) \otimes c_{j} \otimes\left(b_{i} \prec d_{j}\right)\right],
\end{aligned}
$$

$(\mathrm{F} 1 \mathrm{c})=(R(x) \otimes \mathrm{id} \otimes \mathrm{id})\left(-r_{\prec, 23} \succ r_{\prec, 12}\right)$

$$
\begin{aligned}
& =\sum_{i, j}\left[-\left(a_{i} * x\right) \otimes\left(a_{j} \succ b_{i}\right) \otimes b_{j}\right] \\
& =\sum_{i, j}\left[-\left(a_{i} \succ x+a_{i} \prec x\right) \otimes\left(a_{j} \succ b_{i}\right) \otimes b_{j}\right],
\end{aligned}
$$

$(\mathrm{F} 1 \mathrm{~d})=(R(x) \otimes \mathrm{id} \otimes \mathrm{id})\left[r_{\prec, 13} \succ\left(r_{\prec, 23}+r_{\succ, 23}\right)\right]$

$$
=\sum_{i, j}\left\{\left(a_{i} \succ x+a_{i} \prec x\right) \otimes\left[a_{j} \otimes\left(b_{i} \succ b_{j}\right)+c_{j} \otimes\left(b_{i} \succ d_{j}\right)\right]\right\},
$$

$(\mathrm{F} 1 \mathrm{e})=(R(x) \otimes \mathrm{id} \otimes \mathrm{id})\left[-\left(r_{\prec, 23}+r_{\succ, 23}\right) \prec r_{\prec, 12}\right]$

$$
=-\sum_{i, j}\left\{\left(a_{i} \succ x+a_{i} \prec x\right) \otimes\left[\left(a_{j} \prec b_{i}\right) \otimes b_{j}+\left(c_{j} \prec b_{i}\right) \otimes d_{j}\right]\right\},
$$

$\left(\mathrm{F}^{\prime}\right)=\left(r_{\prec, 23}+r_{\succ, 23}\right) \prec\left[\left(\mathrm{id} \otimes L_{\prec}(x) \otimes \mathrm{id}\right) r_{\prec, 12}\right]$

$$
\left.=\sum_{i, j} a_{i} \otimes\left[a_{j} \prec\left(x \succ b_{i}\right) \otimes b_{j}+c_{j} \prec\left(x \succ b_{i}\right)\right] \otimes d_{j}\right],
$$

$(\mathrm{F} 3 \mathrm{a})=\left(\mathrm{id} \otimes \mathrm{id} \otimes L_{\succ}(x)\right)\left(-r_{\prec, 12} * r_{\prec, 13}\right)=\sum_{i, j}-a_{i} * a_{j} \otimes b_{i} \otimes\left(x \succ b_{j}\right)$

$$
=\sum_{i, j}-\left[\left(a_{i} \succ a_{j}+a_{i} \prec a_{j}\right) \otimes b_{i} \otimes\left(x \succ b_{j}\right),\right.
$$

$(\mathrm{F} 3 \mathrm{~b})=\left(\mathrm{id} \otimes \mathrm{id} \otimes L_{\succ}(x)\right)\left(-r_{\prec, 13} \prec r_{\succ, 23}\right)=\sum_{i, j}-\left[a_{i} \otimes c_{j} \otimes x \succ\left(b_{i} \prec d_{j}\right)\right]$,

$(\mathrm{F} 3 \mathrm{c})=\left(\mathrm{id} \otimes \mathrm{id} \otimes L_{\succ}(x)\right)\left(r_{\prec, 23} \succ r_{\prec, 12}\right)=\sum_{i, j}\left[a_{i} \otimes\left(a_{j} \succ b_{i}\right) \otimes\left(x \succ b_{j}\right)\right]$,

$(\mathrm{F} 3 \mathrm{~d})=-\left[\left(\mathrm{id} \otimes \mathrm{id} \otimes L_{\succ}(x)\right) r_{13}\right] \succ\left(r_{\succ, 23}+r_{\prec, 23}\right)$

$$
=-\sum_{i, j} a_{i} \otimes\left[a_{j} \otimes\left(x \succ b_{i}\right) \succ b_{j}+c_{j} \otimes\left(x \succ b_{i}\right) \succ d_{j}\right] .
$$

It is obvious that

$$
\begin{aligned}
& (\mathrm{F} 1)=(\mathrm{F} 1 \mathrm{a})+(\mathrm{F} 1 \mathrm{~b})+(\mathrm{F} 1 \mathrm{c})+(\mathrm{F} 1 \mathrm{~d})+(\mathrm{F} 1 \mathrm{e}), \\
& (\mathrm{F} 2)=(\mathrm{F} 2)^{\prime},
\end{aligned}
$$




$$
(\mathrm{F} 3)=(\mathrm{F} 3 \mathrm{a})+(\mathrm{F} 3 \mathrm{~b})+(\mathrm{F} 3 \mathrm{c})+(\mathrm{F} 3 \mathrm{~d}) .
$$

Therefore eq. (70) holds if only if eq. (67) holds.

Combining Propositions 4.3.1 and 4.3.3, we obtain the following conclusion.

Theorem 4.3.4. Let $(A, \succ, \prec)$ be a dendriform algebra and $r_{\succ}, r_{\prec} \in A \otimes A$. Then the linear maps $\Delta_{\succ}, \Delta_{<}$defined by eqs. (61) and (62) induce a dendriform algebra structure on $A^{*}$ such that $\left(A, A^{*}\right)$ is a dendriform D-bialgebra if and only if $r_{\succ}$ and $r_{\prec}$ satisfy eqs. (63)-(65) and (70)-(72).

Definition 4.3.5. A dendriform D-bialgebra $\left(A, A^{*}\right)$ is called coboundary if its structure is given by $r_{\succ}, r_{\prec} \in A \otimes A$ through Theorem 4.3.4.

Theorem 4.3.6. Let $\left(A, A^{*}, \Delta_{\succ}, \Delta_{\prec}, \beta_{\succ}, \beta_{\prec}\right)$ be a dendriform D-bialgebra. Then there is a canonical dendriform bialgebra structure on the direct sum $A \oplus A^{*}$ of the underlying vector spaces of $A$ and $A^{*}$ such that both the inclusions $i_{1}: A \rightarrow$ $A \oplus A^{*}$ and $i_{2}: A^{*} \rightarrow A \oplus A^{*}$ into the two summands are homomorphisms of dendriform D-bialgebras, where the dendriform D-bialgebra structure on $A^{*}$ is given in Example 4.2.7.

Proof. Let $r=\sum_{i} e_{i} \otimes e_{i}^{*} \in A \otimes A^{*} \subset\left(A \oplus A^{*}\right) \otimes\left(A \oplus A^{*}\right)$ which corresponds to the identity map id: $A \rightarrow A$, where $\left\{e_{1}, \ldots, e_{n}\right\}$ is a basis of $A$ and $\left\{e_{1}^{*}, \ldots, e_{n}^{*}\right\}$ is its dual basis. Suppose that the dendriform D-bialgebra structure " $\succ, \prec$ ” on $A \oplus A^{*}$ is given by

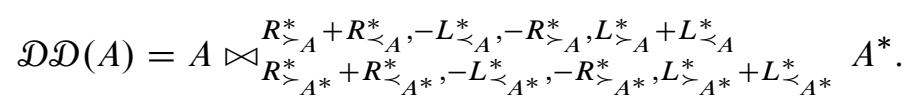

Then we have, for any $x, y \in A, a, b \in A^{*}$,

$$
\begin{gathered}
x \succ y=x \succ_{A} y, \quad x \prec y=x \prec_{A} y, \quad x \succ a=R_{A}^{*}(x) a-L_{\prec_{A^{*}}}^{*}(a) x, \\
x \prec a=-R_{\succ_{A}}^{*}(x) a+L_{A^{*}}^{*}(a) x, \quad a \succ x=R_{A^{*}}^{*}(a) x-L_{\prec_{A}}^{*}(x) a, \\
a \prec x=-R_{\succ_{A^{*}}}^{*}(a) x+L_{A}^{*}(x) a, \quad a \succ b=a \succ_{A^{*}} b, \quad a \prec b=a \prec_{A^{*}} b .
\end{gathered}
$$

If $r_{\succ}=r$ and $r_{\prec}=-r$ satisfies eqs. (63)-(65) and (70)-(72), then

$$
\begin{aligned}
& \Delta_{\mathcal{D D}, \succ}(u)=\left(\mathrm{id} \otimes L(u)-R_{\prec}(u) \otimes \mathrm{id}\right)\left(r_{\succ}\right), \\
& \Delta_{\mathcal{D} D}, \prec(u)=\left(\mathrm{id} \otimes L_{\prec}(u)-R(x) \otimes \mathrm{id}\right)\left(r_{\prec}\right),
\end{aligned}
$$

for all $u \in \mathscr{D} D(A)$, can induce a dendriform D-bialgebra structure on $\mathscr{D} \mathscr{D}(A)$.

In fact, we have

$$
r_{\prec}+r_{\succ}=0, \quad r_{\prec}+\sigma\left(r_{\succ}\right)=\sum_{i}\left(-e_{i} \otimes e_{i}^{*}+e_{i}^{*} \otimes e_{i}\right) .
$$


Therefore eq. (64) holds automatically. By a similar proof as of Theorem 2.3.6, it follows that eqs. (63) and (65) hold and

$$
\begin{aligned}
r_{12} * r_{13}-r_{13} \prec r_{23}-r_{23} \succ r_{12} & =-r_{23} * r_{12}+r_{12} \prec r_{13}+r_{13} \succ r_{23} \\
& =-r_{13} * r_{23}+r_{23} \prec r_{12}+r_{12} \succ r_{13} \\
& =0 .
\end{aligned}
$$

So eqs. (70)-(72) are satisfied. Hence $\mathscr{D} D(A)$ is a dendriform D-bialgebra. Furthermore, for $e_{k} \in A$, we have

$$
\begin{aligned}
\Delta_{\mathscr{D D}, \succ}\left(e_{k}\right) & =\sum_{i}\left[e_{i} \otimes e_{k} * e_{i}^{*}-\left(e_{i} \prec e_{k}\right) \otimes e_{i}^{*}\right] \\
& =\sum_{i, j}\left\langle e_{k}, e_{i}^{*} \succ e_{j}^{*}\right\rangle e_{i} \otimes e_{j} \\
& =\Delta_{\succ}\left(e_{k}\right), \\
\Delta_{\mathcal{D D}, \prec}\left(e_{k}\right) & =\sum_{i}\left[-e_{i} \otimes e_{k} \prec e_{i}^{*}+\left(e_{i} * e_{k}\right) \otimes e_{i}^{*}\right] \\
& =\sum_{i, j}\left\langle e_{k}, e_{i}^{*} \prec e_{j}^{*}\right\rangle e_{i} \otimes e_{j} \\
& =\Delta_{\prec}\left(e_{k}\right) .
\end{aligned}
$$

Therefore the inclusion $i_{1}: A \rightarrow A \oplus A^{*}$ is a homomorphism of dendriform Dbialgebras. Similarly, the inclusion $i_{2}: A^{*} \rightarrow A \oplus A^{*}$ is also a homomorphism of dendriform D-bialgebras, where the dendriform D-bialgebra structure on $A^{*}$ is given in Example 4.2.7.

Definition 4.3.7. Let $\left(A, A^{*}\right)$ be a dendriform D-bialgebra. With the dendriform D-bialgebra structure given in Theorem 4.3.6, $A \oplus A^{*}$ is called a dendriform double of $A$. We denote it by $\mathscr{D} D(A)$.

Corollary 4.3.8. Let $\left(A, A^{*}\right)$ be a dendriform D-bialgebra. Then the dendriform double $\mathscr{D} D(A)$ of $A$ is a dendriform D-bialgebra and the bilinear form $\omega$ given by eq. (7) is a Connes cocycle.

We would like to point out here that, unlike the symmetry of 1-cocycles of $A$ and $A^{*}$ appearing in the definition of a dendriform D-bialgebra $\left(A, A^{*}\right)$, it is not necessary that $\beta$ is also a 1-coboundary of $A^{*}$ for a coboundary dendriform Dbialgebra $\left(A, A^{*}, \Delta_{\succ}, \Delta_{\prec}, \beta_{\succ}, \beta_{\prec}\right)$, where $\Delta_{\succ}, \Delta_{\prec}$ are given by eqs. (61) and (62).

4.4. The $D$-equation and its properties. In this subsection, we consider some simple and special cases to satisfy the eqs. (63)-(65) and (70)-(72).

At first, due to eq. (63), we consider the condition

$$
r_{\prec}=r, \quad r_{\succ}=-\sigma(r), \quad r \in A \otimes A .
$$


Corollary 4.4.1. Let $(A, \succ, \prec)$ be a dendriform algebra and $r=\sum_{i} a_{i} \otimes b_{i} \in A \otimes A$. Then the maps $\Delta_{\succ}, \Delta_{\prec}$ defined by eqs. (61) and (62) with $r_{\succ}, r_{\prec}$ satisfying eq. (73) induce a dendriform algebra structure on $A^{*}$ such that $\left(A, A^{*}\right)$ is a dendriform $D$-bialgebra if and only if $r$ satisfies the following equations.

$$
\begin{gathered}
{\left[P(x \succ y)-\left(\operatorname{id} \otimes L_{\succ}(x)\right) P(y)\right](r-\sigma(r))=0,} \\
\sigma(P(x)) P(y)(r-\sigma(r))=0,
\end{gathered}
$$

$\left(R(x) \otimes \mathrm{id} \otimes \mathrm{id}-\mathrm{id} \otimes \mathrm{id} \otimes L_{\succ}(x)\right)\left[\left(r_{12} * r_{13}-r_{13} \prec r_{32}-r_{23} \succ r_{12}\right)\right.$

$$
+\sum_{i}\left(a_{i} * x\right) \otimes P\left(b_{i}\right)(r-\sigma(r))-a_{i} \otimes\left[P\left(x \succ b_{i}\right)(r-\sigma(r))\right]=0,
$$

$\left(R_{\prec}(x) \otimes \mathrm{id} \otimes \mathrm{id}-\mathrm{id} \otimes \mathrm{id} \otimes L_{\prec}(x)\right)\left(-r_{23} * r_{21}+r_{21} \prec r_{13}+r_{31} \succ r_{23}\right)=0$,

$\left(R_{\prec}(u) \otimes \mathrm{id} \otimes \mathrm{id}-\mathrm{id} \otimes \mathrm{id} \otimes L(u)\right)\left(-r_{31} * r_{32}+r_{32} \prec r_{21}+r_{12} \succ r_{31}\right)$

$$
+\sum_{i}\left[P\left(b_{i}\right)(r-\sigma(r)) \otimes x * a_{i}-P\left(b_{i} \prec x\right)(r-\sigma(r)) \otimes a_{i}\right]=0,
$$

where $x, y \in A, P(x)=\mathrm{id} \otimes L_{\succ}(x)-R_{\prec}(x) \otimes \mathrm{id}$.

Remark 4.4.2. Let $\sigma_{123}, \sigma_{132}: A \otimes A \otimes A \rightarrow A \otimes A \otimes A$ be two linear maps given by

$$
\sigma_{123}(x \otimes y \otimes z)=z \otimes x \otimes y, \quad \sigma_{132}(x \otimes y \otimes z)=y \otimes z \otimes x
$$

for all $x, y, z \in A$. Then we have

$$
\begin{aligned}
& \left(r_{23} * r_{21}-r_{21} \prec r_{13}-r_{31} \succ r_{23}\right)=\sigma_{123}\left(r_{12} * r_{13}-r_{13} \prec r_{32}-r_{23} \succ r_{12}\right), \\
& \left(r_{31} * r_{32}-r_{32} \prec r_{21}-r_{12} \succ r_{31}\right)=\sigma_{132}\left(r_{12} * r_{13}-r_{13} \prec r_{32}-r_{23} \succ r_{12}\right) .
\end{aligned}
$$

Remark 4.4.3. We can also consider the case $r_{\succ}+r_{\prec}=0$, as we did in the proof of Theorem 4.3.6. Obviously, if in addition, $r_{\prec}=r$ is symmetric, then we are in the case satisfying eq. (73).

The simplest way to satisfy eqs. (74)-(78) is to assume that $r$ is symmetric and

$$
r_{12} * r_{13}=r_{13} \prec r_{23}+r_{23} \succ r_{12}
$$

Corollary 4.4.4. Let $(A, \succ, \prec)$ be a dendriform algebra and $r \in A \otimes A$. Suppose that $r$ is symmetric and $r$ satisfies eq. (79). Then the maps $\Delta_{\succ}, \Delta_{\prec}$ defined by eqs. (61) and (62) with $r_{\succ}=-r, r_{\prec}=r$ induce a dendriform algebra structure on $A^{*}$ such that $\left(A, A^{*}\right)$ is a dendriform D-bialgebra.

Definition 4.4.5. Let $(A, \succ, \prec)$ be a dendriform algebra and $r \in A \otimes A$. Eq. (79) is called $D$-equation in $A$. 
By Remark 4.4.2, when $r$ is symmetric, the equivalent forms of the $D$-equation are given as

$$
r_{23} * r_{12}=r_{12} \prec r_{13}+r_{13} \succ r_{23} \quad \text { or } \quad r_{13} * r_{23}=r_{23} \prec r_{12}+r_{12} \succ r_{13} \text {. }
$$

By a similar proof as of Proposition 2.4.4, we have the following conclusion.

Proposition 4.4.6. Let $(A, \succ, \prec)$ be a dendriform algebra and let $r \in A \otimes A$ be $a$ symmetric solution of the D-equation in $A$. Then the dendriform algebra structure and its associated associative algebra structure on the dendriform double $\mathscr{D D}(A)$ is given from the products in A as follows:

$$
\begin{aligned}
a^{*} \prec b^{*} & =-R_{\succ}^{*}\left(r\left(a^{*}\right)\right) b^{*}+L^{*}\left(r\left(b^{*}\right)\right) a^{*}, \\
a^{*} \succ b^{*} & =R^{*}\left(r\left(a^{*}\right)\right) b^{*}-L_{\prec}^{*}\left(r\left(b^{*}\right)\right) a^{*}, \\
a^{*} * b^{*} & =a^{*} \succ b^{*}+a^{*} \prec b^{*}=R_{\prec}^{*}\left(r\left(a^{*}\right)\right) b^{*}+L_{\succ}^{*}\left(r\left(b^{*}\right)\right) a^{*}, \\
x \succ a^{*} & =x \succ r\left(a^{*}\right)-r\left(R^{*}(x) a^{*}\right)+R^{*}(x) a^{*}, \\
x \prec a^{*} & =x \prec r\left(a^{*}\right)+r\left(R_{\succ}^{*}(x) a^{*}\right)-R_{\succ}^{*}(x) a^{*}, \\
x * a^{*} & =x * r\left(a^{*}\right)-r\left(R_{\prec}^{*}(x) a^{*}\right)+R_{\prec}^{*}(x) a^{*} ; \\
a^{*} \succ x & =r\left(a^{*}\right) \succ x+r\left(L_{\prec}^{*}(x) a^{*}\right)-L_{\prec}^{*}(x) a^{*}, \\
a^{*} \prec x & =r\left(a^{*}\right) \prec x-r\left(L^{*}(x) a^{*}\right)+L^{*}(x) a^{*}, \\
a^{*} * x & =r\left(a^{*}\right) * x-r\left(L_{\succ}^{*}(x) a^{*}\right)+L_{\succ}^{*}(x) a^{*}
\end{aligned}
$$

for any $x \in A, a^{*}, b^{*} \in A^{*}$.

Theorem 4.4.7. Let $(A, \succ, \prec)$ be a dendriform algebra and $r \in A \otimes A$. Suppose that $r$ is symmetric and non-degenerate. Then $r$ is a solution of the $D$-equation in $A$ if and only if the inverse of the isomorphism $A^{*} \rightarrow A$ induced by $r$, regarded as $a$ bilinear form $\mathscr{B}$ on $A$ (that is, $\mathcal{B}(x, y)=\left\langle r^{-1} x, y\right\rangle$ for any $x, y \in A$ ) satisfies

$$
\mathscr{B}(x * y, z)=\mathscr{B}(y, z \prec x)+\mathscr{B}(x, y \succ z) \text { for all } x, y, z \in A .
$$

Proof. Let $r=\sum_{i} a_{i} \otimes b_{i}$. Since $r$ is symmetric, $r\left(v^{*}\right)=\sum_{i}\left\langle v^{*}, a_{i}\right\rangle b_{i}=$ $\sum_{i}\left\langle v^{*}, b_{i}\right\rangle a_{i}$ for any $v^{*} \in A^{*}$. Since $r$ is non-degenerate for any $x, y, z \in A$, there exist $u^{*}, v^{*}, w^{*} \in A^{*}$ such that $x=r\left(u^{*}\right), y=r\left(v^{*}\right), z=r\left(w^{*}\right)$. Therefore

$$
\begin{aligned}
\mathcal{B}(x * y, z) & =\left\langle r\left(u^{*}\right) * r\left(v^{*}\right), w^{*}\right\rangle \\
& =\sum_{i, j}\left\langle u^{*}, b_{i}\right\rangle\left\langle v^{*}, b_{j}\right\rangle\left\langle w^{*}, a_{i} * a_{j}\right\rangle=\left\langle w^{*} \otimes u^{*} \otimes v^{*}, r_{12} * r_{13}\right\rangle, \\
\mathcal{B}(y, z \prec x) & =\left\langle v^{*}, r\left(w^{*}\right) \prec r\left(u^{*}\right)\right\rangle \\
& =\sum_{i, j}\left\langle u^{*}, b_{i}\right\rangle\left\langle w^{*}, b_{i}\right\rangle\left\langle v^{*}, a_{i} \prec a_{j}\right\rangle=\left\langle w^{*} \otimes u^{*} \otimes v^{*}, r_{13} \prec r_{23}\right\rangle, \\
\mathcal{B}(x, y \succ z) & =\left\langle r\left(v^{*}\right) \succ r\left(w^{*}\right), u^{*}\right\rangle \\
& =\sum_{i, j}\left\langle v^{*}, b_{i}\right\rangle\left\langle w^{*}, b_{j}\right\rangle\left\langle u^{*}, a_{i} \succ a_{j}\right\rangle=\left\langle w^{*} \otimes u^{*} \otimes v^{*}, r_{23} \succ r_{12}\right\rangle .
\end{aligned}
$$


Therefore $\mathcal{B}$ satisfies eq. (81) if and only if $r$ is a solution of the $D$-equation in $A$.

Definition 4.4.8. Let $(A, \succ, \prec)$ be a dendriform algebra. A bilinear form $\mathcal{B}$ on $\mathrm{A}$ is called a 2 -cocycle if $\mathcal{B}$ satisfies eq. (81).

Remark 4.4.9. Let $\mathcal{B}$ be 2 -cocycle on a dendriform algebra $(A, \succ, \prec)$. Then it is easy to show that $\omega(x, y)=\mathscr{B}(x, y)-\mathscr{B}(y, x)$ (for any $x, y \in A$ ) is a Connes cocycle of the associated associative algebra $(A, *)$. On the other hand, $\mathcal{B}$ satisfies

$$
\mathscr{B}(x \cdot y, z)-\mathscr{B}(x, y \cdot z)=\mathscr{B}(y \cdot x, z)-\mathscr{B}(y, x \cdot z)
$$

for all $x, y, z \in A$, where $x \cdot y=x \succ y-y \prec x$ for any $x, y \in A$. Furthermore, $(A, \cdot)$ is a pre-Lie algebra (see Sections 5.2 and 5.3) and a bilinear form on a pre-Lie algebra $A$ satisfying eq. (82) is called a 2-cocycle on $A$ ([Ku2]). Moreover, a pre-Lie algebra $A$ over the real number field $\mathbb{R}$ is called Hessian if there exists a symmetric and positive definite 2-cocycle on $A$. In geometry, a Hessian manifold $M$ is a flat affine manifold provided with a Hessian metric $g$, that is, $g$ is a Remanning metric such that for any each point $p \in M$ there exists a $C^{\infty}$-function $\varphi$ defined on a neighborhood of $p$ such that $g_{i j}=\frac{\partial^{2} \varphi}{\partial x^{i} \partial x^{j}}$. A Hessian pre-Lie algebra corresponds to an affine Lie group $G$ with a $G$-invariant Hessian metric ([Sh]). Therefore a symmetric and positive definite 2-cocycle on a real dendriform algebra can give a Hessian structure.

Corollary 4.4.10. Let $\left(A, \succ_{A}, \prec_{A}\right)$ be a dendriform algebra and let $r \in A \otimes A$ be a non-degenerate symmetric solution of the D-equation in A. Suppose the dendriform algebra structure " $\succ_{A^{*}}, \prec_{A^{*}}$ " on $A^{*}$ is induced by $r$ via Proposition 4.4.6. Then we have

$$
a^{*} \succ_{A^{*}} b^{*}=r^{-1}\left(r\left(a^{*}\right) \succ_{A} r\left(b^{*}\right)\right), \quad a^{*} \prec_{A^{*}} b^{*}=r^{-1}\left(r\left(a^{*}\right) \prec_{A} r\left(b^{*}\right)\right)
$$

for all $a^{*}, b^{*} \in A^{*}$. Therefore, $r: A^{*} \rightarrow A$ is an isomorphism of dendriform algebras.

Proof. The conclusion can be obtained by a similar proof as of Corollary 2.4.6.

Theorem 4.4.11. Let $(A, \succ, \prec)$ be a dendriform algebra and $r \in A \otimes A$ symmetric. Then $r$ is a solution of the $D$-equation in $A$ if and only if $r$ satisfies

$$
r\left(a^{*}\right) * r\left(b^{*}\right)=r\left(R_{\prec}^{*}\left(r\left(a^{*}\right)\right) b^{*}+L_{\succ}^{*}\left(r\left(b^{*}\right)\right) a^{*}\right) \quad \text { for all } a^{*}, b^{*} \in A^{*} .
$$

Proof. The conclusion can be obtained by a similar proof as of Theorem 2.4.7.

Combining Theorem 4.4.11 and Theorem 3.1.2, we have the following conclusion.

Corollary 4.4.12. Let $(A, \succ, \prec)$ be a dendriform algebra and $r \in A \otimes A$ symmetric. Then $r$ is a solution of the $D$-equation in $A$ if and only if $r$ is an $\mathcal{O}$-operator of the 
associated associative algebra $(A, *)$ associated to $\left(R_{\prec}^{*}, L_{\succ}^{*}\right)$. Therefore there is a dendriform algebra structure on $A^{*}$ given by

$$
a^{*} \succ b^{*}=R_{\prec}^{*}\left(r\left(a^{*}\right)\right) b^{*}, \quad a^{*} \prec b^{*}=L_{\succ}^{*}\left(r\left(b^{*}\right)\right) a^{*}
$$

for all $a^{*}, b^{*} \in A^{*}$. It has the same associated associative algebra as the dendriform algebra on $A^{*}$ given by eq. (80), which is induced by $r$ in the sense of coboundary dendriform D-bialgebras. If $r$ is non-degenerate, then there is a new compatible dendriform algebra structure on A given by

$$
x \succ^{\prime} y=r\left(R_{\prec}^{*}(x) r^{-1} y\right), \quad x \prec^{\prime} y=r\left(L_{\succ}^{*}(y) r^{-1} x\right) \text { for all } x, y \in A,
$$

which is just the dendriform algebra structure given by

$$
\mathscr{B}\left(x \succ^{\prime} y, z\right)=\mathscr{B}(y, z * x), \quad \mathscr{B}\left(x \prec^{\prime} y, z\right)=\mathscr{B}(x, y * z) \quad \text { for all } x, y, z \in A,
$$

where $\mathcal{B}$ is the symmetric 2-cocycle on $A$ induced by $r^{-1}$.

Theorem 4.4.13. Let $(A, *)$ be an associative algebra and $(l, r, V)$ a bimodule. Let $\left(r^{*}, l^{*}, V^{*}\right)$ be the bimodule of $A$ given by Lemma 2.1.2. Suppose that $T: V \rightarrow A$ is an $\mathcal{O}$-operator associated to $(l, r, V)$. Then $r=T+\sigma(T)$ is a symmetric solution of the D-equation in $T(V) \ltimes_{r^{*}, 0,0, l^{*}} V^{*}$, where $T(V) \subset A$ is a dendriform algebra given by eq. (33) and $\left(r^{*}, 0,0, l^{*}\right)$ is a bimodule since its associated associative algebra $T(V)$ is an associative subalgebra of $A$, and $T$ can be identified with an element in $T(V) \otimes V^{*} \subset\left(T(V) \ltimes_{r^{*}, 0,0, l^{*}} V^{*}\right) \otimes\left(T(V) \ltimes_{r^{*}, 0,0, l^{*}} V^{*}\right)$.

Proof. Let $\left\{e_{1}, \ldots, e_{n}\right\}$ be a basis of $A$. Let $\left\{v_{1}, \ldots, v_{m}\right\}$ be a basis of $V$ and $\left\{v_{1}^{*}, \ldots, v_{m}^{*}\right\}$ its dual basis. Set $T\left(v_{i}\right)=\sum_{k=1}^{n} a_{i k} e_{k}, i=1, \ldots, m$. Then

$$
\begin{aligned}
T & =\sum_{i=1}^{m} T\left(v_{i}\right) \otimes v_{i}^{*} \\
& =\sum_{i=1}^{m} \sum_{k=1}^{n} a_{i k} e_{k} \otimes v_{i}^{*} \in T(V) \otimes V^{*} \\
& \subset\left(T(V) \ltimes_{r^{*}, 0,0, l^{*}} V^{*}\right) \otimes\left(T(V) \ltimes_{r^{*}, 0,0, l^{*}} V^{*}\right) .
\end{aligned}
$$

Therefore we have

$$
\begin{gathered}
r_{12} * r_{13}=\sum_{i, j=1}^{m}\left\{T\left(v_{i}\right) * T\left(v_{j}\right) \otimes v_{i}^{*} \otimes v_{j}^{*}+r^{*}\left(T\left(v_{i}\right)\right) v_{j}^{*} \otimes v_{i}^{*} \otimes T\left(v_{j}\right)\right. \\
\left.\quad+l^{*}\left(T\left(v_{j}\right)\right) v_{i}^{*} \otimes T\left(v_{i}\right) \otimes v_{j}^{*}\right\}, \\
r_{13} \prec r_{23}=\sum_{i, j=1}^{m}\left\{v_{i}^{*} \otimes v_{j}^{*} \otimes T\left(v_{i}\right) \prec T\left(v_{j}\right)+T\left(v_{i}\right) \otimes v_{j}^{*} \otimes l^{*}\left(T\left(v_{j}\right)\right) v_{i}^{*}\right\}, \\
r_{23} \succ r_{12}=\sum_{i, j=1}^{m}\left\{T\left(v_{j}\right) \otimes r^{*}\left(T\left(v_{i}\right)\right) v_{j}^{*} \otimes v_{i}^{*}+v_{j}^{*} \otimes T\left(v_{i}\right) \succ T\left(v_{j}\right) \otimes v_{i}^{*}\right\} .
\end{gathered}
$$


On the other hand, we have

$$
\begin{aligned}
\sum_{i, j=1} r^{*}\left(T\left(v_{i}\right)\right) v_{j}^{*} \otimes v_{i}^{*} \otimes T\left(v_{j}\right) & =\sum_{i, j=1} v_{j}^{*} \otimes v_{i}^{*} \otimes T\left(r\left(T\left(v_{i}\right)\right) v_{j}\right), \\
\sum_{i, j=1} l^{*}\left(T\left(v_{j}\right)\right) v_{i}^{*} \otimes T\left(v_{i}\right) \otimes v_{j}^{*} & =\sum_{i, j=1} v_{i}^{*} \otimes T\left(l\left(T\left(v_{j}\right)\right) v_{i}\right) \otimes v_{j}^{*}, \\
\sum_{i, j=1} T\left(v_{i}\right) \otimes v_{j}^{*} \otimes l^{*}\left(T\left(v_{j}\right)\right) v_{i}^{*} & =\sum_{i, j=1} T\left(l\left(T\left(v_{j}\right)\right) v_{i}\right) \otimes v_{j}^{*} \otimes v_{i}^{*}, \\
\sum_{i, j=1} T\left(v_{j}\right) \otimes r^{*}\left(T\left(v_{i}\right)\right) v_{j}^{*} \otimes v_{i}^{*} & =\sum_{i, j=1} T\left(r\left(T\left(v_{i}\right)\right) v_{j}\right) \otimes v_{j}^{*} \otimes v_{i}^{*} .
\end{aligned}
$$

Since $T$ is an $\mathcal{O}$-operator of $A$ associated to $(l, r, V)$ and

$$
T(u) \succ T(v)=T(l(T(u)) v), \quad T(u) \prec T(v)=T(r(T(v)) u)
$$

for all $u, v \in V$, it follows that $r$ is a symmetric solution of the $D$-equation in $T(V) \ltimes_{r^{*}, 0,0, l^{*}} V^{*}$.

Remark 4.4.14. Roughly speaking, a symmetric solution of the $D$-equation corresponds to the symmetric part of an $\mathcal{O}$-operator, whereas an antisymmetric solution of associative Yang-Baxter equation corresponds to the antisymmetric part of an $\mathcal{O}$-operator.

Corollary 4.4.15. Let $(A, \succ, \prec)$ be a dendriform algebra. Then

$$
r=\sum_{i=1}^{n}\left(e_{i} \otimes e_{i}^{*}+e_{i}^{*} \otimes e_{i}\right)
$$

is a symmetric solution of the $D$-equation in $A \ltimes_{R_{\prec}^{*}, 0,0, L_{\succ}^{*}} A^{*}$, where $\left\{e_{1}, \ldots, e_{n}\right\}$ is a basis of $A$ and $\left\{e_{1}^{*}, \ldots, e_{n}^{*}\right\}$ is its dual basis. Moreover, $r$ is non-degenerate and the induced 2-cocycle $\mathcal{B}$ on $A \ltimes_{R_{\prec}^{*}, 0,0, L_{\succ}^{*}} A^{*}$ is given by eq. (2).

Proof. Let $V=A, l=L_{\succ}, r=R_{\prec}$ and $T=$ id in Theorem 4.4.13. Then the conclusion follows immediately.

Remark 4.4.16. A comparison with Theorem 4.3.6 shows that (the non-symmetric) $T=\sum_{i=1}^{n} e_{i} \otimes e_{i}^{*}$ induces a dendriform D-bialgebra structure on

$$
A \ltimes_{R^{*},-L_{\prec}^{*},-R_{\succ}^{*}, L^{*}} A^{*},
$$

whereas the above (symmetric) $r=T+\sigma(T)$ induces a dendriform D-bialgebra structure on $A \ltimes_{R_{\prec}^{*}, 0,0, L_{\succ}^{*}} A^{*}$.

Recall that two Connes cocycles $\left(A_{1}, \omega_{1}\right)$ and $\left(A_{2}, \omega_{2}\right)$ are isomorphic if and only if there exists an isomorphism of associative algebras $\varphi: A_{1} \rightarrow A_{2}$ such that

$$
\omega_{1}(x, y)=\varphi^{*} \omega_{2}(x, y)=\omega_{2}(\varphi(x), \varphi(y)) \text { for all } x, y \in A_{1} .
$$

By a similar proof as of Theorem 2.4.9, we have the following conclusion. 
Theorem 4.4.17. Let $(A, \succ, \prec)$ be a dendriform algebra. Then, as Connes cocycles of associative algebras, the double construction of Connes cocycle (or the dendriform Dbialgebra $)\left(T(A)=A \bowtie A^{*}, \omega\right)$ given by a symmetric solution $r$ of the $D$-equation in $A$ and the double construction of Connes cocycle (or the dendriform D-bialgebra) $\left(T(A)=A \ltimes_{R_{\prec}^{*}, L_{\succ}^{*}} A^{*}, \omega\right)$ are isomorphic, where $\omega$ is given by eq. (7). However, in general, they are not isomorphic as double constructions of Connes cocycles (or dendriform D-bialgebras).

Corollary 4.4.18. Let $(A, \succ, \prec)$ be a dendriform algebra. Then as Connes cocycles of associative algebras, the double constructions of Connes cocycles given by all symmetric solutions of the D-equation in A are isomorphic to the double construction of the Connes cocycle $\left(T(A)=A \ltimes_{R_{\prec}^{*}, L_{\succ}^{*}} A^{*}, \omega\right)$ given by the zero solution.

\section{Comparison (duality) between bialgebra structures}

5.1. Comparison (duality) between antisymmetric infinitesimal bialgebras and dendriform D-bialgebras. The results in the previous sections allow us to compare antisymmetric infinitesimal bialgebras and dendriform D-bialgebras in terms of the following properties: 1-cocycles of associative algebras, matched pairs of associative algebras, associative algebra structures on the direct sum of the associative algebras in the matched pairs, bilinear forms on the direct sum of the associative algebras in the matched pairs, double structures on the direct sum of the associative algebras in the matched pairs, algebraic equations associated to coboundary cases, non-degenerate solutions, $\mathcal{O}$-operators of associative algebras and constructions from dendriform algebras. We list them in Table 1. From this table, we observe that there is a clear analogy between them and in particular, double constructions of Frobenius algebras correspond to double constructions of Connes cocycles in this sense. Moreover, due to the correspondences between certain symmetries and antisymmetries appearing in the Table 1, we regard it as a kind of duality.

Next we consider the case that a dendriform D-bialgebra is also an antisymmetric infinitesimal bialgebra.

Theorem 5.1.1. Let $\left(A, A^{*}, \Delta_{\succ}, \Delta_{\prec}, \beta_{\succ}, \beta_{\prec}\right)$ be a dendriform D-bialgebra. Then $\left(A, A^{*}\right)$ is an antisymmetric infinitesimal bialgebra if and only if the following two equations hold:

$$
\begin{gathered}
\left\langle L_{\succ_{A^{*}}}^{*}\left(b^{*}\right) y, L_{\succ_{A}}^{*}(x) a^{*}\right\rangle=\left\langle R_{\succ_{A^{*}}}^{*}\left(a^{*}\right) x, R_{\succ_{A}}^{*}(y) b^{*}\right\rangle, \\
\left\langle L_{\succ_{A^{*}}}^{*}\left(b^{*}\right) y, R_{\succ_{A}}^{*}(x) a^{*}\right\rangle+\left\langle L_{\succ_{A^{*}}}^{*}\left(a^{*}\right) x, R_{\succ_{A}}^{*}(y) b^{*}\right\rangle \\
=\left\langle R_{\succ_{A^{*}}}^{*}\left(b^{*}\right) x, L_{\succ_{A}}^{*}(y) a^{*}\right\rangle+\left\langle R_{\succ_{A^{*}}}^{*}\left(a^{*}\right) y, L_{\succ_{A}}^{*}(x) b^{*}\right\rangle
\end{gathered}
$$

for any $x, y \in A^{*}, a^{*}, b^{*} \in A^{*}$. 
Table 1. Comparison between antisymmetric infinitesimal bialgebras and dendriform D-bialgebras.

\begin{tabular}{|c|c|c|}
\hline Algebras & $\begin{array}{c}\text { Antisymmetric } \\
\text { infinitesimal bialgebras }\end{array}$ & Dendriform D-bialgebras \\
\hline $\begin{array}{l}1 \text {-cocycles of } \\
\text { associative algebras }\end{array}$ & $(\mathrm{id} \otimes L, R \otimes \mathrm{id})$ & $\begin{array}{c}\left.\text { (id } \otimes L_{\succ}, R \otimes \mathrm{id}\right), \\
\left(\mathrm{id} \otimes L, R_{\prec} \otimes \mathrm{id}\right)\end{array}$ \\
\hline $\begin{array}{l}\text { Matched pairs of } \\
\text { associative algebras }\end{array}$ & $\left(A, A^{*}, R_{A}^{*}, L_{A}^{*}, R_{A^{*}}^{*}, L_{A^{*}}^{*}\right)$ & $\left(A, A^{*}, R_{\prec_{A}}^{*}, L_{\succ_{A}}^{*}, R_{\prec_{A^{*}}}^{*}, L_{\succ_{A^{*}}}^{*}\right)$ \\
\hline $\begin{array}{l}\text { Associative algebra } \\
\text { structures on the } \\
\text { direct sum of the } \\
\text { associative algebras in } \\
\text { the matched pairs }\end{array}$ & $\begin{array}{l}\text { double constructions of } \\
\text { Frobenius algebras }\end{array}$ & $\begin{array}{l}\text { double constructions of Connes } \\
\text { cocycles }\end{array}$ \\
\hline \multirow{3}{*}{$\begin{array}{l}\text { Bilinear forms on the } \\
\text { direct sum of the } \\
\text { associative algebras in } \\
\text { the matched pairs }\end{array}$} & symmetric & antisymmetric \\
\hline & $\begin{array}{c}\left\langle x+a^{*}, y+b^{*}\right\rangle= \\
\left\langle x, b^{*}\right\rangle+\left\langle a^{*}, y\right\rangle\end{array}$ & $\begin{array}{l}\left\langle x+a^{*}, y+b^{*}\right\rangle= \\
-\left\langle x, b^{*}\right\rangle+\left\langle a^{*}, y\right\rangle\end{array}$ \\
\hline & invariant & Connes cocycles \\
\hline $\begin{array}{l}\text { Double structures on } \\
\text { the direct sum of the } \\
\text { associative algebras in } \\
\text { the matched pairs }\end{array}$ & associative doubles & dendriform doubles \\
\hline \multirow{2}{*}{$\begin{array}{l}\text { Algebraic equations } \\
\text { associated to } \\
\text { coboundary cases }\end{array}$} & antisymmetric solutions & symmetric solutions \\
\hline & $\begin{array}{l}\text { associative Yang-Baxter } \\
\text { equations }\end{array}$ & $\begin{array}{l}D \text {-equations in dendriform } \\
\text { algebras }\end{array}$ \\
\hline $\begin{array}{l}\text { Non-degenerate } \\
\text { solutions }\end{array}$ & $\begin{array}{l}\text { Connes cocycles of } \\
\text { associative algebras }\end{array}$ & $\begin{array}{l}\text { 2-cocycles of dendriform } \\
\text { algebras }\end{array}$ \\
\hline \multirow{2}{*}{$\begin{array}{l}\mathcal{O} \text {-operators of } \\
\text { associative algebras }\end{array}$} & associated to $\left(R^{*}, L^{*}\right)$ & associated to $\left(R_{\prec}^{*}, L_{\succ}^{*}\right)$ \\
\hline & antisymmetric parts & symmetric parts \\
\hline \multirow{2}{*}{$\begin{array}{l}\text { Constructions from } \\
\text { dendriform algebras }\end{array}$} & $\begin{array}{c}r= \\
\sum_{i=1}^{n}\left(e_{i} \otimes e_{i}^{*}-e_{i}^{*} \otimes e_{i}\right)\end{array}$ & $r=\sum_{i=1}^{n}\left(e_{i} \otimes e_{i}^{*}+e_{i}^{*} \otimes e_{i}\right)$ \\
\hline & $\begin{array}{l}\text { induced bilinear forms } \\
\left\langle x+a^{*}, y+b^{*}\right\rangle= \\
-\left\langle x, b^{*}\right\rangle+\left\langle a^{*}, y\right\rangle\end{array}$ & $\begin{array}{c}\text { induced bilinear forms } \\
\left\langle x+a^{*}, y+b^{*}\right\rangle= \\
\left\langle x, b^{*}\right\rangle+\left\langle a^{*}, y\right\rangle\end{array}$ \\
\hline
\end{tabular}

Proof. The conclusion can be obtained by a similar proof as of Proposition 2.2.2.

Corollary 5.1.2. Let $(A, \succ, \prec)$ be a dendriform algebra and let $r \in A \otimes A$ be a symmetric solution of the D-equation in A. Suppose the dendriform algebra structure on $A^{*}$ is induced by $r$ from eq. (80). Then $\left(A, A^{*}\right)$ is an antisymmetric infinitesimal bialgebra if and only if the following two equations hold:

$$
\begin{aligned}
& \left\langle y \prec_{A}\left(x \succ_{A} r\left(a^{*}\right)\right)-y *_{A} r\left(R_{\succ_{A}}^{*}(x) a^{*}\right), b^{*}\right\rangle \\
& \quad=\left\langle r\left(L_{\prec_{A}}^{*}(y) b^{*}\right) *_{A} x-\left(r\left(b^{*}\right) \prec_{A} y\right) \succ x, a^{*}\right\rangle ;
\end{aligned}
$$




$$
\begin{aligned}
&\left\langle y \prec_{A}\left(r\left(a^{*}\right) \prec_{A} x\right)\right.-\left(y \succ_{A} r\left(a^{*}\right)\right) \succ_{A} x \\
&\left.+r\left(R_{\succ_{A}}^{*}(y) a^{*}\right) *_{A} x-y *_{A} r\left(L_{\prec_{A}}^{*}(x) a^{*}\right), b^{*}\right\rangle \\
&=\left\langle-x \prec_{A}\left(r\left(b^{*}\right) \prec_{A} y\right)+\left(x \succ_{A} r\left(b^{*}\right)\right) \succ_{A} y-r\left(R_{\succ_{A}}^{*}(x) a^{*}\right) *_{A} y\right. \\
&\left.+x *_{A} r\left(L_{\prec_{A}}^{*}(y) a^{*}\right), a^{*}\right\rangle,
\end{aligned}
$$

for any $x, y \in A$ and $a^{*} \in A^{*}$.

Corollary 5.1.3. Let $\left(A, A^{*}, \Delta_{\succ}, \Delta_{\prec}, \beta_{\succ}, \beta_{\prec}\right)$ be a dendriform D-bialgebra. If eqs. (84) and (85) are satisfied, then there are two associative algebra structures $A \bowtie_{R_{\prec_{A}}^{*}, L_{\succ_{A}}^{*}, L_{A^{*}}^{*}}^{R^{*}} A^{*}$ and $A \bowtie_{R_{A^{*}}^{*}, L_{A^{*}}^{*}}^{R_{*}^{*}, L_{A}^{*}} A^{*}$ on the direct sum $A \oplus A^{*}$ of the underlying vector spaces of $A$ and $A^{*}$ such that both $A$ and $A^{*}$ are associative subalgebras and the bilinear form given by eq. (7) is a Connes cocycle on $A \bowtie_{R_{\prec_{A}}^{*}, L_{A^{*}}^{*}, L_{\succ_{A}}^{*} A^{*}}^{*} A^{*}$ and the bilinear form given by eq. (2) is invariant on $A \bowtie_{R_{A}^{*}, L_{A^{*}}^{*}}^{R^{*}, L_{A}^{*}} A^{*}$. Moreover, these two associative algebras are not isomorphic in general.

Example 5.1.4. Let $\left(A, *_{A}\right)$ be an associative algebra and let $\omega$ be a Connes cocycle on $\left(A, *_{A}\right)$. Then there is an antisymmetric infinitesimal bialgebra whose associative algebra structure on $A^{*}$ is given by a non-degenerate solution $r$ of the associative Yang-Baxter equation as follows:

$$
\Delta(x)=(\mathrm{id} \otimes L(x)-R(x) \otimes \mathrm{id}) r
$$

for all $x \in A$, where $r: A^{*} \rightarrow A$ is given by $\omega(x, y)=\left\langle r^{-1}(x), y\right\rangle$. On the other hand, there exists a compatible dendriform algebra structure " $\succ_{A}, \prec_{A}$ " on $A$ given by eq. (53), that is,

$$
\omega\left(x \succ_{A} y, z\right)=\omega\left(y, z *_{A} x\right), \quad \omega\left(x \prec_{A} y, z\right)=\omega\left(x, y *_{A} z\right)
$$

for all $x, y, z \in A$. Moreover, there exists a compatible dendriform algebra structure on the associative algebra $A^{*}$ given by

$$
a^{*} \succ_{A^{*}} b^{*}=r^{-1}\left(r\left(a^{*}\right) \succ_{A} r\left(b^{*}\right)\right), \quad a^{*} \prec_{A^{*}} b^{*}=r^{-1}\left(r\left(a^{*}\right) \prec_{A} r\left(b^{*}\right)\right),
$$

for all $a^{*}, b^{*} \in A$. Furthermore, it is easy to show that

$$
\begin{aligned}
& L_{\succ_{A}}^{*}(x) a^{*}=r^{-1}\left(r\left(a^{*}\right) *_{A} x\right), \\
& R_{\succ_{A}}^{*}(x) a^{*}=-r^{-1}\left(x \prec_{A} r\left(a^{*}\right)\right), \\
& L_{\succ_{A}}^{*}(x) a^{*}=-r^{-1}\left(r\left(a^{*}\right) \succ_{A} x\right), \\
& R_{\prec_{A}}^{*}(x) a^{*}=r^{-1}\left(x * r\left(a^{*}\right)\right), \\
& L_{\succ_{A^{*}}}^{*}\left(a^{*}\right) x=x *_{A} r\left(a^{*}\right), \\
& R_{\succ_{A^{*}}}^{*}\left(a^{*}\right) x=-r\left(a^{*}\right) \prec_{A} x, \\
& L_{\prec_{A^{*}}}^{*}\left(a^{*}\right) x=-x \succ_{A} r\left(a^{*}\right), \\
& R_{\prec_{A^{*}}}^{*}\left(a^{*}\right) x=r\left(a^{*}\right) *_{A} x
\end{aligned}
$$


for all $x \in A, a^{*} \in A^{*}$. Therefore, by Theorem 4.2.4, $\left(A, A^{*}\right)$ (as dendriform algebras) is a dendriform D-bialgebra if and only if $\left(A, A^{*}, R_{\succ_{A}}^{*}, L_{\succ_{A}}^{*}, R_{\succ_{A}}^{*}, L_{\succ_{A^{*}}}^{*}\right)$ is a matched pair of associative algebras, which is the case if and only if $A$ is 2-step nilpotent, that is, $x *_{A} y *_{A} z=0$ for any $x, y, z \in A$. In this case, by eq. (86), it is equivalent to

$$
x \succ_{A}\left(y \succ_{A} z\right)=x \prec_{A}\left(y \prec_{A} z\right)=x \succ_{A}\left(y \prec_{A} z\right)=0
$$

for all $x, y, z \in A$. Therefore, under such conditions, eqs. (84) and (85) hold naturally.

\subsection{Duality in the version of Lie algebras: Lie bialgebras and pre-Lie bialge-} bras. There is a similar duality for Lie algebras, which was presented in [Bai2]. In order to be self-contained, we give a brief introduction in this subsection. We would like to point out that, although we give the Lie bialgebras and pre-Lie bialgebras as structures similar to antisymmetric infinitesimal bialgebras and dendriform D-bialgebras, here, in fact, it is the Manin triples (Lie bialgebras) that have been first studied and then motivate us to study the other structures.

There are two kinds of important (non-degenerate) bilinear forms on Lie algebras. A bilinear form $\mathscr{B}($, ) on a Lie algebra $A$ is invariant if

$$
\mathscr{B}([x, y], z)=\mathscr{B}(x,[y, z]) \text { for all } x, y \in A .
$$

A 2-cocycle (symplectic form) on a Lie algebra $A$ is an antisymmetric bilinear from $\omega$ satisfying

$$
\omega([x, y], z)+\omega([y, z], x)+\omega([z, x], y)=0 \quad \text { for all } x, y, z \in A .
$$

Moreover, the algebras that play a role similar to dendriform algebras in the double constructions of Frobenius algebras and Connes cocycles are pre-Lie algebras. In fact, pre-Lie algebras (or under other names like left-symmetric algebras, quasi-associative algebras, Vinery algebras and so on) are a class of natural algebraic systems appearing in many fields in mathematics and mathematical physics (see the survey article [Bu] and the references therein).

Definition 5.2.1. Let $A$ be a vector space over a field $\mathbb{F}$ with a bilinear product $(x, y) \rightarrow x y . A$ is called a pre-Lie algebra if

$$
(x y) z-x(y z)=(y x) z-y(x z) \text { for all } x, y, z \in A .
$$

Let $A$ be a pre-Lie algebra. For any $x, y \in A$, let $L(x)$ and $R(x)$ denote the left and right multiplication operator, respectively, that is, $L(x)(y)=x y, R(x)(y)=y x$. Let $L: A \rightarrow \operatorname{gl}(A)$ with $x \rightarrow L(x)$ and $R: A \rightarrow \operatorname{gr}(A)$ with $x \rightarrow R(x)$ (for every $x \in A$ ) be two linear maps. For a Lie algebra $\mathscr{E}$, we let ad $(x)$ denote the adjoint operator, that is, $\operatorname{ad}(x) y=[x, y]$, and ad: $\mathscr{G} \rightarrow \operatorname{gl}(\mathscr{E})$ with $x \rightarrow \operatorname{ad}(x)$ is a linear map. 
Proposition 5.2.2. Let $A$ be a pre-Lie algebra.

(1) The commutator

$$
[x, y]=x y-y x \quad \text { for all } x, y \in A
$$

defines a Lie algebra $\mathscr{E}(A)$, which is called the sub-adjacent Lie algebra of $A$ and $A$ is also called a compatible pre-Lie algebra structure on the Lie algebra $\mathcal{G}(A)$.

(2) The map $L: A \rightarrow \operatorname{gl}(A)$ gives a representation of the Lie algebra $\mathcal{E}(A)$.

Proposition 5.2.3 ([Chu]). Let $\mathcal{E}$ be a Lie algebra and let $\omega$ be a non-degenerate 2-cocycle on $\mathcal{G}$ (such a Lie algebra called a symplectic Lie algebra). Then there exists a compatible pre-Lie algebra structure on $\mathcal{E}$ defined by

$$
\omega(x * y, z)=-\omega(y,[x, z]) \text { for all } x, y, z \in \mathcal{E} \text {. }
$$

Next we give the "double constructions" of Lie algebras with non-degenerate invariant bilinear forms or non-degenerate 2-cocycles. In fact, both of them have their own (independent) interest in many fields.

At first, recall that $(\mathcal{H}, \mathscr{H}, \rho, \mu)$ is a matched pair of Lie algebras if $\mathscr{E}$ and $\mathscr{H}$ are Lie algebras and $\rho: \mathscr{E} \rightarrow \mathfrak{g l}(\mathscr{H})$ and $\mu: \mathscr{H} \rightarrow \mathrm{gl}(\mathscr{E})$ are representations satisfying

$$
\begin{aligned}
\rho(x)[a, b]-[\rho(x) a, b]-[a, \rho(x) b]+\rho(\mu(a) x) b-\rho(\mu(b) x) a & =0, \\
\mu(a)[x, y]-[\mu(a) x, y]-[x, \mu(a) y]+\mu(\rho(x) a) y-\mu(\rho(y) a) x & =0,
\end{aligned}
$$

for any $x, y \in \mathcal{G}$ and $a, b \in \mathscr{H}$. In this case, there exists a Lie algebra structure on the direct sum $\mathscr{H} \oplus \mathscr{H}$ of the underlying vector spaces of $\mathcal{G}$ and $\mathscr{H}$ given by

$$
[x+a, y+b]=[x, y]+\mu(a) y-\mu(b) x+[a, b]+\rho(x) b-\rho(y) a
$$

for all $x, y \in \mathscr{H}, a, b \in \mathscr{H}$. We denote it by $\mathscr{G} \bowtie_{\mu}^{\rho} \mathscr{H}$ or simply $\mathscr{Y} \bowtie \mathscr{H}$. Moreover, every Lie algebra which is the direct sum of the underlying vector spaces of two subalgebras can be obtained from a matched pair of Lie algebras as above.

Definition 5.2.4. Let $\mathcal{G}$ be a Lie algebra. Suppose that there is a Lie algebra structure on the direct sum of the underlying vector spaces of $\mathcal{G}$ and its dual space $\mathscr{E}^{*}$ such that $\mathcal{E}$ and $\mathcal{E}^{*}$ are Lie subalgebras.

(a) If the natural symmetric bilinear form on $\mathscr{\Theta} \oplus \mathcal{E}^{*}$ given by eq. (2) is invariant, then $\left(\mathscr{E} \bowtie \mathscr{E}^{*}, \mathcal{E}, \mathscr{E}^{*}\right)$ is called a (standard) Manin triple.

(b) If the natural antisymmetric bilinear form on $\mathscr{\mathcal { G }} \oplus \mathcal{E}^{*}$ given by eq. (7) is a 2cocycle, then it is called a phase space of the Lie algebra $\mathcal{E}([\mathrm{Ku} 1])$. ( $\left.\mathscr{\bigotimes} \bowtie \mathscr{E}^{*}, \mathcal{E}, \mathscr{E}^{*}\right)$ is also called a para-Kähler structure on the Lie algebra $\mathscr{\mathcal { G } ^ { * }} \mathcal{E}^{*}$ ([Kan]).

For a Lie algebra $\mathcal{E}$ and a representation $(\rho, V)$ of $\mathcal{E}$, recall that a 1-cocycle $T$ associated to $\rho$ (and denoted by $(\rho, T)$ ) is a linear map from $\mathcal{E}$ to $V$ satisfying

$$
T([x, y])=\rho(x) T(y)-\rho(y) T(x) \text { for all } x, y \in \mathcal{E} .
$$


Definition 5.2.5. (a) Let $\mathscr{E}$ be a Lie algebra. A Lie bialgebra structure on $\mathscr{E}$ is an antisymmetric linear map $\delta: \mathscr{E} \rightarrow \mathscr{E} \otimes \mathscr{E}$ such that $\delta^{*}: \mathscr{E}^{*} \otimes \mathscr{E}^{*} \rightarrow \mathscr{E}^{*}$ is a Lie bracket on $\mathscr{E}^{*}$ and $\delta$ is a 1 -cocycle of $\mathscr{E}$ associated to ad $\otimes$ id $+\mathrm{id} \otimes$ ad with values in $\mathscr{G} \otimes \mathscr{E}$. We denote it by $\left(\mathscr{E}, \mathscr{E}^{*}\right)$ or $(\mathscr{E}, \delta)$.

(b) Let $A$ be a vector space. A pre-Lie bialgebra structure on $A$ is a pair of linear maps $(\Delta, \beta)$ such that $\Delta: A \rightarrow A \otimes A, \beta: A^{*} \rightarrow A^{*} \otimes A^{*}$ and

(1) $\Delta^{*}: A^{*} \otimes A^{*} \rightarrow A^{*}$ defines a pre-Lie algebra structure on $A^{*}$,

(2) $\beta^{*}: A \otimes A \rightarrow A$ defines a pre-Lie algebra structure on $A$,

(3) $\Delta$ is a 1-cocycle of $\mathscr{E}(A)$ associated to $L \otimes \mathrm{id}+\mathrm{id} \otimes$ ad with values in $A \otimes A$,

(4) $\beta$ is a 1-cocycle of $\mathscr{G}\left(A^{*}\right)$ associated to $L \otimes \mathrm{id}+\mathrm{id} \otimes$ ad with values in $A^{*} \otimes A^{*}$.

We denote it by $\left(A, A^{*}, \Delta, \beta\right)$ or simply $\left(A, A^{*}\right)$.

Theorem 5.2.6. (a) Let $\left(\mathscr{G},[,]_{\mathscr{E}}\right)$ and $\left(\mathscr{E}^{*},[,]_{\mathscr{E}}\right)$ be two Lie algebras. Then the following conditions are equivalent:

(1) $\left(\mathscr{E} \bowtie \mathscr{E}^{*}, \mathcal{E}, \mathscr{E}^{*}\right)$ is a standard Manin triple with the bilinear form (2).

(2) $\left(\mathscr{E}, \mathscr{E}^{*}, \mathrm{ad}_{\mathscr{g}}^{*}, \mathrm{ad}_{\mathscr{G}^{*}}^{*}\right)$ is a matched pair of Lie algebras.

(3) $\left(\mathscr{G}, \mathscr{G}^{*}\right)$ is a Lie bialgebra.

(b) Let $(A, \cdot)$ and $\left(A^{*}, \circ\right)$ be two pre-Lie algebras. Then the following conditions are equivalent:

(1) $\left(\mathscr{E}(A) \bowtie \mathscr{E}(A)^{*}, \mathcal{E}(A), \mathcal{E}\left(A^{*}\right)\right)$ is a para-Kähler Lie algebra with the bilinear form (7).

(2) $\left(\mathscr{G}(A), \mathscr{Y}\left(A^{*}\right), L_{.}^{*}, L_{\circ}^{*}\right)$ is a matched pair of Lie algebras.

(3) $\left(A, A^{*}\right)$ is a pre-Lie bialgebra.

In fact, a Lie bialgebra is the Lie algebra $\mathcal{E}$ of a Poisson-Lie group $G$ equipped with additional structures induced from the Poisson structure on $G$, and a Poisson-Lie group is a Lie group with a Poisson structure compatible with the group operation in a certain sense. Poisson-Lie groups play an important role in symplectic geometry and quantum group theory (cf. [D] and the references therein). On the other hand, in geometry, a para-Kähler manifold is a symplectic manifold with a pair of transversal Lagrangian foliations ([Li]). A para-Kähler Lie algebra $\mathcal{E}$ is the Lie algebra of a Lie group $G$ with a $G$-invariant para-Kähler structure ([Kan]).

We have already obtained many properties of Lie bialgebras and pre-Lie algebras which are similar to our study in the previous sections. We collect them in the Appendix and we compare pre-Lie bialgebras and Lie bialgebras in terms of their certain properties in Table 2. From Table 2, we observe that there is also a clear analogy between them and in particular, due to the correspondences between certain symmetries and antisymmetries appearing in the analogy, we can regard it as a kind of duality again which is similar to the duality appearing in the Table 1. 
Table 2. Comparison between Lie bialgebras and pre-Lie bialgebras.

\begin{tabular}{|c|c|c|}
\hline Algebras & Lie bialgebras & Pre-Lie bialgebras \\
\hline $\begin{array}{l}\text { Corresponding Lie } \\
\text { groups }\end{array}$ & Poisson-Lie groups & para-Kähler Lie groups \\
\hline $\begin{array}{l}\text { 1-cocycles of Lie } \\
\text { algebras }\end{array}$ & $\mathrm{id} \otimes \mathrm{ad}+\mathrm{ad} \otimes \mathrm{id}$ & $L \otimes \mathrm{id}+\mathrm{id} \otimes \mathrm{ad}$ \\
\hline $\begin{array}{l}\text { Matched pairs of Lie } \\
\text { algebras }\end{array}$ & $\left(\mathcal{G}, \mathscr{E}^{*}, \mathrm{ad}_{\mathscr{G}}^{*}, \mathrm{ad}_{\mathscr{G}^{*}}^{*}\right)$ & $\left(\mathcal{E}(A), \mathcal{E}\left(A^{*}\right), L_{A}^{*}, L_{A^{*}}^{*}\right)$ \\
\hline $\begin{array}{l}\text { Lie algebra structures } \\
\text { on the direct sum of the } \\
\text { Lie algebras in the } \\
\text { matched pairs }\end{array}$ & Manin triples & phase spaces \\
\hline \multirow{3}{*}{$\begin{array}{l}\text { Bilinear forms on the } \\
\text { direct sum of the Lie } \\
\text { algebras in the matched } \\
\text { pairs }\end{array}$} & symmetric & antisymmetric \\
\hline & $\begin{array}{c}\left\langle x+a^{*}, y+b^{*}\right\rangle= \\
\left\langle x, b^{*}\right\rangle+\left\langle a^{*}, y\right\rangle\end{array}$ & $\begin{array}{l}\left\langle x+a^{*}, y+b^{*}\right\rangle= \\
-\left\langle x, b^{*}\right\rangle+\left\langle a^{*}, y\right\rangle\end{array}$ \\
\hline & invariant & 2-cocycles \\
\hline $\begin{array}{l}\text { Double structures on } \\
\text { the direct sum of the } \\
\text { Lie algebras in the } \\
\text { matched pairs }\end{array}$ & Drinfeld doubles & symplectic doubles \\
\hline \multirow{2}{*}{$\begin{array}{l}\text { Algebraic equations } \\
\text { associated to coboundary } \\
\text { cases }\end{array}$} & antisymmetric solutions & symmetric solutions \\
\hline & $\begin{array}{l}\text { classical Yang-Baxter } \\
\text { equations in Lie algebras }\end{array}$ & $S$-equations in pre-Lie algebras \\
\hline \multirow{2}{*}{ Non-degenerate solutions } & 2-cocycles of Lie algebras & 2-cocycles of pre-Lie algebras \\
\hline & symplectic structures & Hessian structures \\
\hline \multirow{2}{*}{$\begin{array}{l}\mathcal{O} \text {-operators of Lie } \\
\text { algebras }\end{array}$} & associated to $\mathrm{ad}^{*}$ & associated to $L^{*}$ \\
\hline & antisymmetric parts & symmetric parts \\
\hline \multirow{2}{*}{$\begin{array}{l}\text { Constructions from } \\
\text { pre-Lie algebras }\end{array}$} & $\begin{array}{c}r= \\
\sum_{i=1}^{n}\left(e_{i} \otimes e_{i}^{*}-e_{i}^{*} \otimes e_{i}\right)\end{array}$ & $r=\sum_{i=1}^{n}\left(e_{i} \otimes e_{i}^{*}+e_{i}^{*} \otimes e_{i}\right)$ \\
\hline & $\begin{array}{l}\text { induced bilinear forms } \\
\left\langle x+a^{*}, y+b^{*}\right\rangle= \\
-\left\langle x, b^{*}\right\rangle+\left\langle a^{*}, y\right\rangle\end{array}$ & $\begin{array}{c}\text { induced bilinear forms } \\
\left\langle x+a^{*}, y+b^{*}\right\rangle= \\
\left\langle x, b^{*}\right\rangle+\left\langle a^{*}, y\right\rangle\end{array}$ \\
\hline
\end{tabular}

\subsection{Relationships among four bialgebras}

Proposition 5.3.1 ([Cha1], [A2]). Let $(A, \succ, \prec)$ be a dendriform algebra. Then there is a pre-Lie algebra structure on $(A, \cdot)$ given by

$$
x \cdot y=x \succ y-y \prec x \text { for all } x, y \in A \text {. }
$$

Corollary 5.3.2. Let $(A, \succ, \prec)$ be a dendriform algebra. Then the sub-adjacent Lie algebra of the pre-Lie algebra $(A, \cdot)$ given by eq. $(88)$ is the same as the commutator Lie algebra of the associated associative algebra $(A, *)$, that is,

$$
[x, y]=x * y-y * x=x \cdot y-y \cdot x=x \succ y+x \prec y-y \succ x-y \prec x
$$


for all $x, y \in A$.

Therefore, as Chapoton pointed out in [Cha1] (also see [A2], [A4], [EMP]), there is the following commutative diagram of categories:

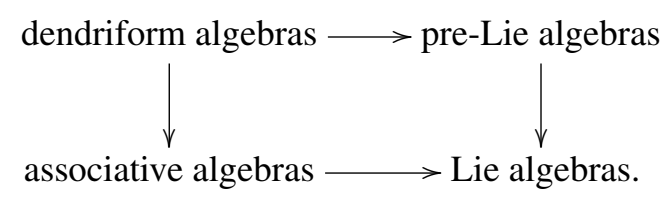

In this diagram, the left vertical arrow is given by eq. (32), the top horizontal arrow is given by eq. (88), the bottom arrow is given by eq. (87) since an associative algebra is a special pre-Lie algebra, and the right vertical arrow is given by eq. (87).

Obviously, if a symmetric or antisymmetric bilinear form on an associative algebra is invariant or a Connes cocycle respectively, then it is also invariant or a 2-cocycle on the commutator Lie algebra respectively,.

Theorem 5.3.3. (1) A double construction of a Frobenius algebra gives a standard Manin triple (on the commutator Lie algebra) naturally.

(2) A double construction of Connes cocycles gives a para-Kähler Lie algebra (on the commutator Lie algebra) naturally.

Corollary 5.3.4. (1) Any antisymmetric infinitesimal bialgebra is a Lie bialgebra (in the sense of its commutator Lie algebra).

(2) Any dendriform D-bialgebra is a pre-Lie bialgebra (in the sense of eq. (88)).

Corollary 5.3.5. We have the following relationship among the antisymmetric infinitesimal bialgebras, dendriform algebras, Lie bialgebras and pre-Lie bialgebras:

dendriform D-bialgebras $\longrightarrow$ pre-Lie bialgebras

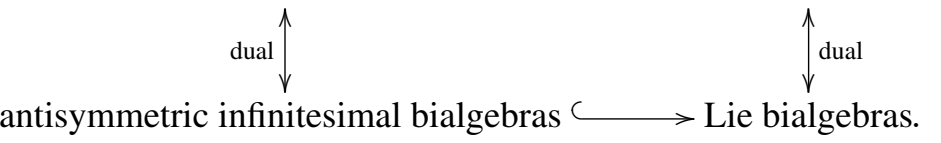

Here $\uparrow$ means the duality given in Sections 5.1 and 5.2, and $\hookrightarrow$ means the inclusion in the sense of Corollary 5.3.4.

Remark 5.3.6. Part (1) of Corollary 5.3.4 and the relation given by the bottom $\hookrightarrow$ in the above diagram were also pointed out in [A3].

Corollary 5.3.7. Let $\left(A, A^{*}, \Delta_{\succ}, \Delta_{\prec}, \beta_{\succ}, \beta_{\prec}\right)$ be a dendriform D-bialgebra. If eqs. (84) and (85) hold, then $\left(A, A^{*}\right)$ is an antisymmetric infinitesimal bialgebra. $\left(A, A^{*}\right)$ is also a pre-Lie bialgebra in the sense of eq. (88). Furthermore, as the commutator Lie algebras, $\left(\mathscr{E}(A), \mathscr{E}(A)^{*}\right)$ is a Lie bialgebra. Therefore, there is an 
associative algebra structure and a Lie algebra structure on the direct sum $A \oplus A^{*}$ of the underlying space of $A$ and $A^{*}$ such that the natural symmetric bilinear form given by eq. (2) is invariant on both of them and the natural antisymmetric bilinear form given by eq. (7) is a Connes cocycle on the associative algebra and a 2-cocycle on the Lie algebra. Moreover, under such a condition, we have the following commutative diagram:

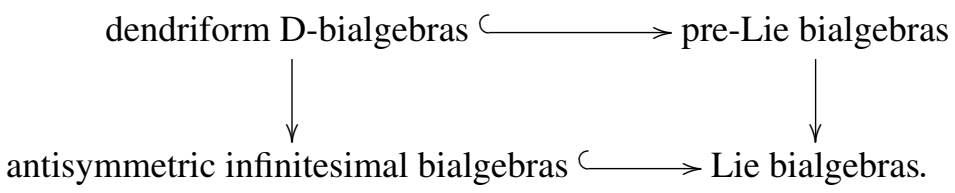

Acknowledgments. The author thanks Professors M. Aguiar, A. Connes, L. Guo and J.-L. Loday for important suggestion. This work was supported in part by NSFC (10621101, 10920161), NKBRPC (2006CB805905) and SRFDP (200800550015).

\section{Appendix: Some properties of Lie bialgebras and pre-Lie bialgebras}

In this appendix, we list some properties of Lie bialgebras and pre-Lie bialgebras. Most of the results can be found in [Bai2] and the references therein.

Proposition A.1. (a) Let $\left(\mathcal{E}, \mathscr{E}^{*}\right)$ be a Lie bialgebra. Then there is a canonical Lie bialgebra structure on $\mathcal{E} \oplus \mathcal{E}^{*}$ such that the inclusions $i_{1}: \mathcal{E} \rightarrow \mathcal{E} \oplus \mathcal{E}^{*}$ and $i_{2}: \mathscr{E}^{*} \rightarrow \mathcal{E} \oplus \mathcal{E}^{*}$ into the two summands are homomorphisms of Lie bialgebras, where the Lie bialgebra structure on $\mathcal{E}^{*}$ is given by $-\delta \mathscr{E}^{*}$. Such a structure is called a classical (Drinfeld) double of $\mathcal{E}$.

(b) Let $\left(A, A^{*}, \Delta, \beta\right)$ be a pre-Lie bialgebra. Then there is a canonical pre-Lie bialgebra structure on $A \oplus A^{*}$ such that both the inclusions $i_{1}: A \rightarrow A \oplus A^{*}$ and $i_{2}: A^{*} \rightarrow A \oplus A^{*}$ into the two summands are homomorphisms of pre-Lie bialgebras. Such a structure is called a symplectic double of $A$.

Definition A.2. (a) A Lie bialgebra $(\mathcal{H}, \delta)$ is called coboundary if $\delta$ is a 1-coboundary of $\mathcal{G}$ associated to ad $\otimes \mathrm{id}+\mathrm{id} \otimes \mathrm{ad}$, that is, there exists an $r \in \mathcal{E} \otimes \mathcal{E}$ such that

$$
\delta(x)=(\operatorname{ad}(x) \otimes \mathrm{id}+\mathrm{id} \otimes \operatorname{ad}(x)) r \quad \text { for all } x \in \mathcal{E} .
$$

(b) A pre-Lie bialgebra $\left(A, A^{*}, \Delta, \beta\right)$ is called coboundary if $\Delta$ is a 1-coboundary of $\mathcal{E}(A)$ associated to $L \otimes \mathrm{id}+\mathrm{id} \otimes \mathrm{ad}$, that is, there exists an $r \in A \otimes A$ such that

$$
\Delta(x)=(L(x) \otimes \mathrm{id}+\mathrm{id} \otimes \operatorname{ad}(x)) r \quad \text { for all } x \in A .
$$

Theorem A.3. (a) Let $\mathcal{E}$ be a Lie algebra and let $r \in \mathcal{E} \otimes \mathcal{E}$. Then the map $\delta: \mathcal{G} \rightarrow \mathcal{E} \otimes \mathcal{E}$ defined by eq. (A.1) induces a Lie bialgebra structure on $\mathcal{E}$ if and only if the following two conditions are satisfied for any $x \in \mathcal{E}$ : 
(1) $(\operatorname{ad}(x) \otimes \mathrm{id}+\mathrm{id} \otimes \operatorname{ad}(x))(r+\sigma(r))=0$,

(2) $(\operatorname{ad}(x) \otimes \mathrm{id} \otimes \mathrm{id}+\mathrm{id} \otimes \operatorname{ad}(x) \otimes \mathrm{id}+\mathrm{id} \otimes \mathrm{id} \otimes \operatorname{ad}(x))\left(\left[r_{12}, r_{13}\right]+\left[r_{12}, r_{23}\right]+\right.$ $\left.\left[r_{13}, r_{23}\right]\right)=0$.

(b) Let $A$ be a pre-Lie algebra and let $r \in A \otimes A$. Then the map $\Delta$ defined by eq. (A.2) induces a pre-Lie algebra structure on $A^{*}$ such that $\left(A, A^{*}\right)$ is a pre-Lie bialgebra if and only if the following two conditions are satisfied for any $x, y \in A$ :

(1) $[P(x \cdot y)-P(x) P(y)](r-\sigma(r))=0$,

(2) $Q(x)[[r, r]]=0$,

where $Q(x)=L(x) \otimes \mathrm{id} \otimes \mathrm{id}+\mathrm{id} \otimes L(x) \otimes \mathrm{id}+\mathrm{id} \otimes \mathrm{id} \otimes \operatorname{ad}(x), P(x)=$ $L(x) \otimes \mathrm{id}+\mathrm{id} \otimes L(x)$ and

$$
[[r, r]]=r_{13} \cdot r_{12}-r_{23} \cdot r_{21}+\left[r_{23}, r_{12}\right]-\left[r_{13}, r_{21}\right]-\left[r_{13}, r_{23}\right]
$$

Corollary A.4. (a) Let $\mathcal{E}$ be a Lie algebra and $r \in \mathscr{E} \otimes \mathscr{E}$. If $r$ is antisymmetric and $r$ satisfies

$$
\left[r_{12}, r_{13}\right]+\left[r_{12}, r_{23}\right]+\left[r_{13}, r_{23}\right]=0,
$$

then the map $\delta: \mathcal{E} \rightarrow \mathcal{E} \otimes \mathcal{E}$ defined by eq. (A.1) induces a Lie bialgebra structure on $\mathcal{E}$.

(b) Let $A$ be a pre-Lie algebra and $r \in A \otimes A$. Suppose that $r$ is symmetric. Then the map $\Delta$ defined by eq. (A.2) induces a pre-Lie algebra structure on $A^{*}$ such that $\left(A, A^{*}\right)$ is a pre-Lie bialgebra if

$$
-r_{12} \cdot r_{13}+r_{12} \cdot r_{23}+\left[r_{13}, r_{23}\right]=0
$$

Definition A.5. (a) Let $\mathcal{E}$ be a Lie algebra and $r \in \mathcal{E} \otimes \mathcal{E}$. Eq. (A.3) is called the classical Yang-Baxter equation in $\mathcal{E}$. in $A$.

(b) Let $A$ be a pre-Lie algebra and $r \in A \otimes A$. Eq. (A.4) is called $S$-equation

Let $\mathcal{E}$ be a Lie algebra and let $\rho: \mathcal{E} \rightarrow \mathfrak{g l}(V)$ be a representation. Recall that a linear map $T: V \rightarrow \mathcal{E}$ is called an $\mathcal{O}$-operator of $\mathcal{E}$ associated to $\rho$ if $T$ satisfies

$$
[T(u), T(v)]=T(\rho(T(u)) v-\rho(T(v)) u) \quad \text { for all } u, v \in V .
$$

Proposition A.6. (a) Let $\mathcal{E}$ be a Lie algebra and $r \in \mathscr{E} \otimes \mathcal{G}$.

(1) Suppose that $r$ is antisymmetric and non-degenerate. Then $r$ is a solution of the classical Yang-Baxter equation in $\mathcal{G}$ if and only if the isomorphism $\mathcal{E}^{*} \rightarrow \mathcal{E}$ induced by $r$, regarded as a bilinear form on $\mathcal{E}$, is a 2-cocycle on $\mathcal{E}$.

(2) Suppose that $r$ is antisymmetric. Then $r$ is a solution of the classical YangBaxter equation in $\mathcal{E}$ if and only if $r$ is an $\mathcal{O}$-operator of $\mathcal{E}$ associated to ad*, that is, $r$ satisfies

$$
\left[r\left(a^{*}\right), r\left(b^{*}\right)\right]=r\left(\operatorname{ad}^{*}\left(r\left(a^{*}\right)\right) b^{*}-\operatorname{ad}^{*}\left(r\left(b^{*}\right)\right) a^{*}\right) \quad \text { for all } a^{*}, b^{*} \in \mathscr{E}^{*} .
$$


(b) Let $A$ be a pre-Lie algebra and $r \in A \otimes A$.

(1) Suppose that $r$ is symmetric and non-degenerate. Then $r$ is a solution of $S$ equation in $A$ if and only if the inverse of the isomorphism $A^{*} \rightarrow A$ induced by $r$, regarded as a bilinear form $B$ on $A$, is a 2-cocycle on $A$ (see eq. (82)).

(2) Suppose that $r$ is symmetric. Then $r$ is a solution of $S$-equation in $A$ if and only if $r$ is an $\mathcal{O}$-operator of $\mathcal{E}(A)$ associated to $L^{*}$, that is, $r$ satisfies

$$
\left[r\left(a^{*}\right), r\left(b^{*}\right)\right]=r\left(L_{.}^{*}\left(r\left(a^{*}\right)\right) b^{*}-L_{.}^{*}\left(r\left(b^{*}\right)\right) a^{*}\right) \text { for all } a^{*}, b^{*} \in A^{*} .
$$

Lemma A.7. Let $\mathcal{E}$ be a Lie algebra and let $\rho: \mathcal{G} \rightarrow \mathrm{g} \mathfrak{L}(V)$ be a representation. Let $T: V \rightarrow \mathcal{E}$ be an $\mathcal{O}$-operator associated to $\rho$. Then the product

$$
u \circ v=\rho(T(u)) v \quad \text { for all } u, v \in V
$$

defines a pre-Lie algebra structure on $V$. Therefore $V$ is a Lie algebra as the subadjacent Lie algebra of this pre-Lie algebra and $T$ is a homomorphism of Lie algebras. Furthermore, $T(V)=\{T(v) \mid v \in V\} \subset \mathcal{E}$ is a Lie subalgebra of $\mathcal{E}$ and there is an induced pre-Lie algebra structure on $T(V)$ given by

$$
T(u) \cdot T(v)=T(u \circ v)=T(\rho(T(u)) v) \text { for all } u, v \in V .
$$

Moreover, its sub-adjacent Lie algebra structure is just the Lie subalgebra structure of $\mathcal{E}$ and $T$ is a homomorphism of pre-Lie algebras.

Proposition A.8. Let $\mathcal{E}$ be a Lie algebra and let $\rho: \mathcal{E} \rightarrow \mathfrak{g} \mathfrak{l}(V)$ be a representation. Let $\rho^{*}: \mathcal{G} \rightarrow \mathfrak{g l}\left(V^{*}\right)$ be the dual representation of $\rho$.

(a) A linear map $T: V \rightarrow \mathcal{E}$ is an $\mathcal{O}$-operator of $\mathcal{E}$ associated to $\rho$ if and only if $r=T-\sigma(T)$ is an antisymmetric solution of the classical Yang-Baxter equation in $\mathcal{G} \propto_{\rho^{*}} V^{*}$.

(b) Let $T: V \rightarrow \mathcal{E}$ be an $\mathcal{O}$-operator associated to $\rho$. Then $r=T+\sigma(T)$ is a symmetric solution of the $S$-equation in $T(V) \ltimes_{\rho^{*}, 0} V^{*}$, where $T(V) \subset \mathcal{G}$ is a pre-Lie algebra given by eq. (A.5) and $\left(\rho^{*}, 0\right)$ is a bimodule since its sub-adjacent Lie algebra $\mathcal{E}(T(V))$ is a Lie subalgebra of $\mathcal{E}$, and $T$ can be identified with an element in $T(V) \otimes V^{*} \subset\left(T(V) \ltimes_{\rho^{*}, 0} V^{*}\right) \otimes\left(T(V) \ltimes_{\rho^{*}, 0} V^{*}\right)$.

Proposition A.9. Let $(A, \cdot)$ be a pre-Lie algebra. Let $\left\{e_{1}, \ldots, e_{n}\right\}$ be a basis of $A$ and $\left\{e_{1}^{*}, \ldots, e_{n}^{*}\right\}$ its dual basis.

(a) $r$ given by eq. (31) is an antisymmetric solution of the classical Yang-Baxter equation in $\mathscr{G}(A) \ltimes_{L^{*}} \mathscr{G}(A)^{*}$. Moreover, $r$ is non-degenerate and the induced 2cocycle $\mathcal{B}$ of $\mathscr{G}(A) \ltimes_{L^{*}} \mathscr{H}(A)^{*}$ is given by eq. (7).

(b) $r$ given by eq. (83) is a symmetric solution of the $S$-equation in $A \ltimes_{L^{*}, 0} A^{*}$. Moreover, $r$ is non-degenerate and the induced 2-cocycle $\mathcal{B}$ of $A \ltimes_{L^{*}, 0} A^{*}$ is given by eq. (2). 
Theorem A.10. Let $\left(A, A^{*}, \Delta, \beta\right)$ be a pre-Lie bialgebra. Then $\left(\mathscr{G}(A), \mathscr{E}\left(A^{*}\right)\right)$ is a Lie bialgebra if and only if

$$
\begin{aligned}
& \left\langle R_{.}^{*}(x) a^{*}, R_{\circ}^{*}\left(b^{*}\right) y\right\rangle+\left\langle R_{.}^{*}(x) b^{*}, R_{\circ}^{*}\left(a^{*}\right) y\right\rangle \\
& \quad=\left\langle R_{.}^{*}(y) b^{*}, R_{\circ}^{*}\left(a^{*}\right) x\right\rangle+\left\langle R_{.}^{*}(y) a^{*}, R_{\circ}^{*}\left(b^{*}\right) x\right\rangle,
\end{aligned}
$$

for any $x, y \in A^{*}, a^{*}, b^{*} \in A^{*}$.

\section{References}

[A1] M. Aguiar, Infinitesimal Hopf algebras. In New trends in Hopf algebra theory (La Falda, 1999), Contemp. Math. 267, Amer. Math. Soc., Providence, RI, 2000, 1-29. Zbl 0982.16028 MR 1800704

[A2] M. Aguiar, Pre-Poisson algebras. Lett. Math. Phys. 54 (2000), 263-277. Zbl 1032.17038 MR 1846958

[A3] M. Aguiar, On the associative analog of Lie bialgebras. J. Algebra 244 (2001), 492-532. Zbl 0991.16033 MR 1859038

[A4] M. Aguiar, Infinitesimal bialgebras, pre-Lie and dendriform algebras. In Hopf algebras, Lecture Notes in Pure and Appl. Math. 237, Marcel Dekker, New York 2004, 1-33. Zbl 1059.16027 MR 2051728

[Bai1] C. Bai, A unified algebraic approach to the classical Yang-Baxter equation. J. Phys. A 40 (2007), 11073-11082. Zbl 1118.17008 MR 2396216

[Bai2] C. Bai, Left-symmetric bialgebras and an analogue of the classical Yang-Baxter equation. Commun. Contemp. Math. 10 (2008), 221-260. Zbl 1173.17025 MR 2409367

[BGN1] C. Bai, L. Guo, and X. Ni, $\mathcal{O}$-operators on associative algebras and associative YangBaxter equations. Preprint 2009; arXiv:0910.3261 [math.RA].

[BGN2] C. Bai, L. Guo, and X. Ni, $\mathcal{O}$-operators on associative algebras and dendriform algebras. Preprint 2010; arXiv:1003.2432 [math.RA].

[BaN] A. A. Balinskiŭ and S. P. Novikov, Poisson brackets of hydrodynamic type, Frobenius algebras and Lie algebras. Dokl. Akad. Nauk SSSR 283 (1985), 1036-1039; English transl. Soviet Math. Dokl. 32 (1985), 228-231. Zbl 0606.58018 MR 802121

[Bax] G. Baxter, An analytic problem whose solution follows from a simple algebraic identity. Pacific J. Math. 10 (1960), 731-742. Zbl 0095.12705 MR 0119224

[Bo] M. Bordemann, Nondegenerate invariant bilinear forms on nonassociative algebras. Acta Math. Univ. Comenian. (N.S.) 66 (1997), 151-201. Zbl 1014.17003 MR 1620480

[BFN] M. Bordemann, T. Filk, and C. Nowak, Algebraic classification of actions invariant under generalized flip moves of two-dimensional graphs. J. Math. Phys. 35 (1994), 4964-4988. Zbl 0822.05026 MR 1290913

[BrN] R. Brauer and C. Nesbitt, On the regular representations of algebras. Proc. Nat. Acad. Sci. U.S.A. 23 (1937), 236-240. Zbl 0016.34102 
[Bu] D. Burde, Left-symmetric algebras, or pre-Lie algebras in geometry and physics. Cent. Eur. J. Math. 4 (2006), 323-357. Zbl 1151.17301 MR 2233854

[Cha1] F. Chapoton, Un endofoncteur de la catégorie des opérades. In Dialgebras and related operads, Lecture Notes in Math. 1763, Springer, Berlin 2001, 105-110. Zbl 0999.17004 MR 1860996

[Cha2] F. Chapoton, Un théorème de Cartier-Milnor-Moore-Quillen pour les bigèbres dendriformes et les algèbres braces. J. Pure Appl. Algebra 168(2002), 1-18. Zbl 0994.18006 MR 1879927

[CP] V. Chari and A. Pressley, A guide to quantum groups. Cambridge University Press, Cambridge 1994. Zbl 0839.17010 MR 1300632

[Chu] B. Y. Chu, Symplectic homogeneous spaces. Trans. Amer. Math. Soc. 197 (1974), 145-159. Zbl 0261.53039 MR 0342642

[C] A. Connes, Non-commutative differential geometry. Inst. Hautes Études Sci. Publ. Math. 62 (1985), 41-144. Zbl 0592.46056 MR 0823176

[D] V. Drinfeld, Hamiltonian structures on Lie groups, Lie bialgebras and the geometric meaning of the classical Yang-Baxter equations. Dokl. Akad. Nauk SSSR 268 (1983), 285-287; English transl. Soviet Math. Dokl. 27 (1983), 68-71. Zbl 0526.58017 MR 0688240

[E1] K. K. Ebrahimi-Fard, Loday-type algebras and the Rota-Baxter relation. Lett. Math. Phys. 61 (2002), 139-147. Zbl 1035.17001 MR 1936573

[E2] K. Ebrahimi-Fard, On the associative Nijenhuis relation. Electron. J. Combin. 11 (2004), no. 1, Research Paper R38. Zbl 1074.17001 MR 2097304

[EMP] K. Ebrahimi-Fard, D. Manchon, and F. Patras, New identities in dendriform algebras. J. Algebra 320 (2008), 708-727. Zbl 1153.17003 MR 2422313

[F1] L. Foissy, Les algèbres de Hopf des arbres enracinés décorés, II. Bull. Sci. Math. 126 (2002), 249-288. Zbl 1013.16027 MR 1909461

[F2] L. Foissy, Bidendriform bialgebras, trees, and free quasi-symmetric functions. J. Pure Appl. Algebra 209 (2007), 439-459. Zbl 1123.16030 MR 2293319

[Fra1] A. Frabetti, Dialgebra homology of associative algebras. C. R. Acad. Sci. Paris Sér. I Math. 325 (1997), 135-140. Zbl 0883.16005 MR 1467065

[Fra2] A. Frabetti, Leibniz homology of dialgebras of matrices. J. Pure Appl. Algebra 129 (1998), 123-141. Zbl 0936.17003 MR 1624446

[Fro] G. Frobenius, Theorie der hyperkomplexen Größen. Sitzber. Königlich Preuss. Akad. Wiss. Berlin 1903 (1903), 504-537; Theorie der hyperkomplexen Größen. II; ibid. 1903 (1903), 634-645. JFM 34.0238.02

[H1] R. Holtkamp, Comparison of Hopf algebras on trees. Arch. Math. (Basel) 80 (2003), 368-383. Zbl 1056.16030 MR 1982837

[H2] R. Holtkamp, On Hopf algebra structures over free operads. Adv. Math. 207 (2006), 544-565. Zbl 1117.16027 MR 2271016

[JR] S. A. Joni and G.-C. Rota, Coalgebras and bialgebras in combinatorics. Stud. Appl. Math. 61 (1979), 93-139. Zbl 0471.05020 MR 544721 
[Kac] V. G. Kac, Infinite dimensional Lie algebras. Progr. Math. 44, Birkhäuser, Boston 1983. Zbl 0537.17001 MR 0739850

[Kan] S. Kaneyuki, Homogeneous symplectic manifolds and dipolarizations in Lie algebras. Tokyo J. Math. 15 (1992), 313-325. Zbl 0781.53027 MR 1197100

[Kap] G. Karpilovsky, Symmetric and G-algebras. Math. Appl. 60, Kluwer Academic Publishers, Dordrecht 1990. Zbl 0705.16001 MR 1065394

[Ko] J. Kock, Frobenius algebras and 2D topological quantum field theories. London Math. Soc. Stud. Texts 59, Cambridge University Press, Cambridge 2004. Zbl 1046.57001 MR 2037238

[Ku1] B. A. Kupershmidt, Non-abelian phase spaces. J. Phys. A 27 (1994), 2801-2809. Zbl 0842.58030 MR 1280835

[Ku2] B. A. Kupershmidt, On the nature of the Virasoro algebra. J. Nonlinear Math. Phys. 6 (1999), 222-245. Zbl 1015.17027 MR 1686317

[Ku3] B. A. Kupershmidt, What a classical $r$-matrix really is. J. Nonlinear Math. Phys. 6 (1999), 448-488. Zbl 1015.17015 MR 1722068

[LS] R. G. Larson and M. E. Sweedler, An associative orthogonal bilinear form for Hopf algebras. Amer. J. Math. 91 (1969), 75-94. Zbl 0179.05803 MR 0240169

[Li] P. Libermann, Sur le problème d'équivalence de certaines structures infinitésimales. Ann. Mat. Pura Appl. (4) 36 (1954), 27-120. Zbl 0056.15401 MR 0066020

[Lo1] J.-L. Loday, Dialgebras. In Dialgebras and related operads, Lecture Notes in Math. 1763, Springer, Berlin 2001, 7-66. Zbl 0999.17002 MR 1860994

[Lo2] J.-L. Loday, Arithmetree. J. Algebra 258 (2002), 275-309. Zbl 1063.16044 MR 1958907

[Lo3] J.-L. Loday, Scindement d'associativité et algèbres de Hopf. In Actes des Journées Mathématiques à la Mémoire de Jean Leray, Sémin. Congr. 9, Soc. Math. France, Paris 2004, 155-172. Zbl 1073.16032 MR 2145941

[Lo4] J.-L. Loday, Generalized bialgebras and triples of operads. Astérisque 320 (2008). Zbl 1178.18001 MR 2504663

[LR1] J.-L. Loday and M. O. Ronco, Hopf algebra of the planar binary trees. Adv. Math. 139 (1998), 293-309. Zbl 0926.16032 MR 1654173

[LR2] J.-L. Loday and M. O. Ronco, Order structure on the algebra of permutations and of planar binary trees. J. Algebraic Combin. 15 (2002), 253-270. Zbl 0998.05013 MR 1900627

[MR1] A. Medina and P. Revoy, Caractérisation des groupes de Lie ayant une pseudométrique bi-invariante. Applications. In Seminaire Sud-Rhodanien en Géométrie III: Journees lyonnaises de la Societe Mathematique de France (Lyon, 1983), Hermann, Paris, Paris 1984, 149-166. Zbl 0539.53039 MR 0753868

[MR2] A. Medina and P. Revoy, Algèbres de Lie et produit scalaire invariant. Ann. Sci. École Norm. Sup. (4) 18 (1985), 553-561. Zbl 0592.17006 MR 826103

[Ron] M. Ronco, Eulerian idempotents and Milnor-Moore theorem for certain noncocommutative Hopf algebras. J. Algebra 254 (2002), 152-172. Zbl 1017.16033 MR 1927436 
[Rot] G.-C. Rota, Baxter operators, an introduction. In Gian-Carlo Rota on combinatorics, Introductory papers and commentaries, Birkhäuser, Boston 1995. Zbl 0841.01031 MR 1392961

[RFFS] I. Runkel, J. Fjelstad, J. Fuchs, and C. Schweigert, Topological and conformal field theory as Frobenius algebras. In Categories in algebra, geometry and mathematical physics, Contemp. Math. 431, Amer. Math. Soc., Providence, RI, 2007, 225-247. Zbl 1154.18006 MR 2342831

[Sc] R. D. Schafer, An introduction to nonassociative algebras. Corrected reprint of the 1966 original, Dover Publications, New York 1995. Zbl 0145.25601 MR 1375235

[Se] M. A. Semenov-Tyan-Shanskii, What is a classical $r$-matrix? Funktsional. Anal. i Prilozh. 17 (1983), no.4, 17-33; English transl. Funct. Anal. Appl. 17 (1983), 259-272. Zbl 0535.58031 MR 0725413

[Sh] H. Shima, Homogeneous Hessian manifolds. Ann. Inst. Fourier (Grenoble) 30 (1980), 91-128. Zbl 0424.53023 MR 597019

[St] A. Stolin, Frobenius algebras and the Yang-Baxter equation. In New symmetries in the theories of fundamental interactions (Karpacz, 1996), Wydawnictwo Naukowe PWN, Warsaw 1997, 93-97. MR 1634712

[U] K. Uchino, Quantum analogy of Poisson geometry, related dendriform algebras and Rota-Baxter operators. Lett. Math. Phys. 85 (2008), 91-109. Zbl 05544981 MR 2443932

[Y] K. Yamagata, Frobenius algebras. In Handbook of algebra, Vol. 1, North-Holland, Amsterdam 1996, 841-887. Zbl 0879.16008 MR 1421820

[Z] V. N. Zhelyabin, Jordan bialgebras and their connection with Lie bialgebras. Algebra i Logika 36 (1997), 3-25; English transl. Algebra Logic 36 (1997), 1-16. Zbl 0935.17014 MR 1454688

Received March 10, 2009

C. Bai, Chern Institute of Mathematics \& LPMC, Nankai University, Tianjin 300071, P.R. China

E-mail: baicm@nankai.edu.cn 